DOE/RL- $-90-48$

DE93 001475

\title{
Hanford Site Single-Shell Tank Roadmap
}

Date Published

April 1991 


\section{EXECUTIVE SUMMARY}

The Hanford Site Singie-Shell Tank Roadmap covers the near-term waste management activities to ensure safe interim storage of 140 million liters of waste. It also addresses the environmental restoration activities to close the 6 single-shell tank operable units, which include 149 single-shell tanks. These tanks were constructed starting in the $1940^{\prime}$ s. Sixty-six tanks have leaked or are assumed to be leaking.

\section{ASSUMPTIONS}

The single-shell tanks will be closed under the Resource Conservation and Recovery Act of 1976 (RCRA) rather than seeking a RCRA operating permit. The preferred closure option decision (i.e., to leave or retrieve) will be resolved through the preparation and completion of a supplemental to the Hanford Defense Waste Environmental Impact Statement.

\section{REGULATORY DRIVERS}

The primary regulatory drivers are the Hanford Federal Facility Agreement and Consent Order (Tri-Party Agreement) and RCRA. These require characterization, interim stabilization, technology development/demonstration, and closure.

\section{MILESTONES}

The following major milestones are taken from the Tri-Party Agreement:

$\begin{array}{lr}\text { Complete single-shell tank interim stabilization } & \text { September } 1995 \\ \text { Develop single-shell tank waste retrieval } & \\ \text { technology and complete scale-model testing } & \text { June } 1994 \\ \text { Initiate full-scale demonstration of waste } & \\ \text { retrieval technology } & \text { October } 1997 \\ \text { Complete analysis of at least two complete core } & \\ \text { samples from each single-shell tank } & \text { September } 1998 \\ \text { Initiate full-scale tank farm demonstration } & \text { June } 2004 \\ \text { project } & \text { June } 2018 \\ \text { Complete closure of all } 149 \text { single-shell tanks } & \end{array}$

\section{LOGIC AND PLANNED ACTIVITIES}

One hundred forty-two million liters of single-shell tank wastes are stored in 149 single-shell tanks. Safety concerns are being evaluated and corrective actions formulated. Characterization is being performed in support of tank safety, remediation, interim stabilization, and isolation, as well as 
closure planning. After the single-shell tank wastes are placed in a safe interim storage configuration, the single-shell tank operable units will be closed under the National Environmental Policy Act/environmental impact statement and RCRA closure processes. The wastes that may be retrieved will be dispositioned. New technology is needed to support each major step in this process.

\section{ISSUES IDENTIFICATION AND ANALYSIS}

The Roadmap process identifies five primary issues and their root causes. These five issues in priority order are:

\begin{tabular}{cl} 
Priority & \multicolumn{1}{c}{ Issue } \\
1 & $\begin{array}{l}\text { Current storage method for waste is not safe or } \\
\text { environmentally sound }\end{array}$ \\
2 & Ability to meet committed closure milestones is uncertain \\
3 & NEPA pathways have not been defined \\
4 & $\begin{array}{l}\text { Regulatory closure requirements (State, EPA, DOE, NRC) do } \\
\text { not agree with HOW-EIS record of decision risk-based } \\
\text { approach }\end{array}$ \\
5 & Technology development process is still evolving
\end{tabular}

The following is a summary of the top priority single-shell tank issue, key sub-issues, desired activities, and schedules to resolve it.

Issue I - Current Storage Method for Waste is not Safe or Environmentally Sound

Safety issues of concern include cyclic hydrogen-oxidizer mixture release and potential combustion, potential ferrocyanide mixture explosion, the presence of flammable organic nitrate-nitrite mixtures, high radioactive decay heat in a tank requiring regular addition of water and potential for toxic vapor releases. Instrumentation and control systems associated with the waste tank system are outdated and inadequate. The waste is not adequately characterized. Maintenance has been deficient and the tanks have exceeded their design iife. Emergency pumping capability is deficient and there is insuffi... cient double-shell tank space to support operations. Water infiltrations into single-shell tanks are possibie. There is no process or criteria to validate single-shell tank data and administrative systems are less than adequate. A formal safety review of all the single-shell tanks must be done. Finally, resolution of these safety issues may affect Tri-Party Agreement mi?estones. 
Analysis and laboratory studies of chemical reactions and mechanisms wiil be completed as follows:

\author{
Hydrogen generation \\ Organic/ritirate/nitrite \\ Ferrocyanide \\ High heat tanks
}

\author{
September 1993 \\ March 1994 \\ September 1993 \\ September 1997
}

Upgrades to safety documentation will be completed by September 1997.

Characterization of the affected tanks will be completed as follows:

Hydrogen, high heat, and ferrocyanide tanks

All tanks will be characterized Organic nitrate/nitrite

September 1996
September 1998
March 1997

Interim remediation and upgrades to the tanks and ancillary systems will continue through 2003. This date is budget constrained and could be up to 5 years earlier for the safety issue tanks if required funding is available each year.

\section{FUTURE DIRECTION}

This Roadmap has highlighted the need for integrated planning and resource allocation. The June 1990 Five-Year Plan did not address the tank safety concerns that evolved since its publication. Potential impacts to TriParty Agreement milestones for characterization, stabilization and isolation, technology development/demonstration, and closure will be examined in greater detail to strengthen the technical decision basis and to minimize consequences. The Roadmap indicates the advantage of accelerating characterization programs, technology evaluations, and supplemental environmental impact statement preparation. Working with regulators and expanded public outreach programs are essential to successful completion of this activity. 
This page intentionally left blank. 
CONTENTS

1.0 INTRODUCTION ....................................

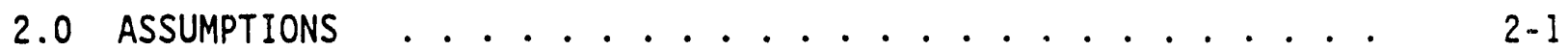

3.0 REgULATORY REQUIREMENTS ........................ . . . . . .

4.0 MILESTONES ........................... 4-1

5.0 PLANNED ACTIVITIES ......................... 5 . I

6.0 LOGIC DIAGRAM ......................... 6-.

7.0 ISSUES ANALYSIS . . . . . . . . . . . . . . . . . . 7-1

7.1 Issue I - Current Storage Method for Waste is not Safe or Environmentally Sound .. . . . . . . . . 7-5

7.2 Issue II - Ability to Meet Committed Closure Milestones is Uncertain ............... . 7-77

7.3 Issue III - NEPA Pathways Have not Been Defined . . . . . 7-111

7.4 Issue IV - Regulatory Closure Requirements (State, EPA, DOE, NRC) do not Agree With HDW-EIS Record of Decision Risk-Based Approach . . . . . . . . . . . 7-123

7.5 Issue V - Technology Development Process is Still Evolving ................ 7-143

8.0 ISSUES RESOLUTION AND DESIRED ACTIVITIES . . . . . . . . . 8-1

9.0 HEADQUARTERS ISSUES ..................... . . $9-1$

10.0 REFERENCES ................................. 10.1 


\section{TABLES}

4-1 Single-Shell Tank Milestones-Milestone Number Order .... . 4-3 4-2 External Single-Shel Tank Commitments ......... . 4-6

\section{FIGURES}

1-1 Single-Shel1 Tank Roadmap Methodology Flow Diagram

4-1 Milestone Diagram for External Single-Shell Tank Commitments

7-1 Issue I - Current Storage Method for Waste is not Safe or

7-2 Issue II - Ability to Meet Committed ciosure Milestones is Uncertain .................... . . 7-79

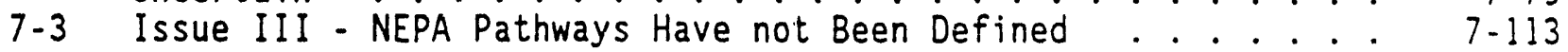

7-4 Issue IV - Regulatory Ciosure Requirements (State, EPA, DOE, NRC) do not Agree with HDW-EIS Record of Decision Risk-Based Approach ................. 7-125

7-5 Issue V - Technology Development Process is Stili Evolving . 7-145 


\section{ACRONYMS}

$\begin{array}{ll}\text { AEA } & \text { Atomic Energy Act of } 1954 \\ \text { ADS } & \text { activity data sheet } \\ \text { ALARA } & \text { as low as reasonably achievable } \\ \text { CERCLA } & \text { Comprehensive Environmental Response Compensation Liability } \\ \text { CFR } & \text { Act of } 1980 \\ \text { DNFSB } & \text { Code of Federal Regulations } \\ \text { DOE } & \text { Defense Nuclear Facility Safety Board } \\ \text { DOE-HQ } & \text { U.S. Department of Energy } \\ \text { DOE-RL } & \text { U.S. Department of Energy-Headquarters } \\ \text { ECOlogy } & \text { U.S. Department of Energy-Richland Operations Office } \\ \text { EIS } & \text { Washington State Department of Ecology } \\ \text { EPA } & \text { environmental impact statement } \\ \text { ERRA } & \text { U.S. Department of Environmental Protection Agency } \\ \text { HDW } & \text { Environmental Restoration and Remedial Action } \\ \text { HDW-EIS } & \text { Hanford Defense Waste } \\ \text { LDR } & \text { Hanford Defense Waste-Environmental Impact Statement } \\ \text { NEPA } & \text { land disposal restrictions } \\ \text { NPDES } & \text { National Environmental Policy Act } \\ \text { NPL } & \text { National Pollutant Discharge Elimination System } \\ \text { NRC } & \text { national priorities list } \\ \text { RCRA } & \text { U.S. Nuclear Regulatory Commission } \\ \text { SEIS } & \text { Resource Conservation and Recovery Act of 1976 } \\ \text { SEPA } & \text { supplemental environmental impact statement } \\ \text { SST } & \text { State Environmental Policy Act } \\ \text { TRU } & \text { single-shell tank } \\ \text { TSD } & \text { transuranic } \\ \text { WAC } & \text { treatment, storage, and disposal } \\ \text { Westinghouse } & \text { Washington Administrative Code } \\ \text { Hanford } & \text { Westinghouse Hanford Company } \\ & \end{array}$


This page intentionally left blank. 
DOE/RL $-90-48$

Predecisional Draft

$4 / 5 / 91$

\subsection{INTRODUCTION}

\subsection{PURPOSE}

The Hanford Site Single-Shell Tank Roadmap (Roadmap) documents a planning process that focuses on the identification and resolution of issues, as shown in Figure 1-1. The Roadmap process consists of three phases: assessment, analysis, and issue resolution. The assessment phase products are the assumptions, regulatory requirements, milestones, planned activities with their associated schedules, and the logic for conducting single-shell tank (SST) activities. The analysis phase products are issue identification and analysis, desired activities schedules, and issue resolution schedules. The issue resolution phase products are modifications to activity data sheets $(A D S)^{*}$, and U.S. Department of Energy-Headquarters (DOE-HQ) issues. As an issue-oriented planning process, the Roadmap identifies issues and the activities needed to resolve those issues.

In addition, this Roadmap will serve as a management and communications tool by identifying issues within the framework of SST activities. Many of the SST issues are common to other Hanford Site issues. The identification and resolution of these issues is of interest to people throughout the Hanford Site.

\subsection{DOCUMENT STRUCTURE}

This Roadmap was prepared by following the guidance contained in the DOE-HQ Environmental Restoration and Waste Management Roadmap Methodology Document (Ref. 1). The structure of this Roadmap document follows the structure specified in the methodology document.

Chapter 2.0, Assumptions, catalogs the basic assumptions used in developing this Roadmap. The assumptions are part of the planning basis for the SST closure scheduled to be completed in 2018 as required by the Hanford Federal Facility Agreen t and Consent Order (Tri-Party Agreement) (Ref. 2). These do not include co ingency as a buffer against unexpected events.

Chapter 3.0, Regulatory Requirements, provides a description of the regulatory requirements for SST activities. Regulations, regulatory authority, requirements, and status are included for all applicable regulations.

"Concurrent roadmap and ADS schedules did not allow preparation of separate ADS modification chapter for this document. However, ADSs preparation was heavily influenced by information from roadmap preparation. 
Figure 1-1. Single-Shell Tank Roadmap Methodology Flow Diagram.

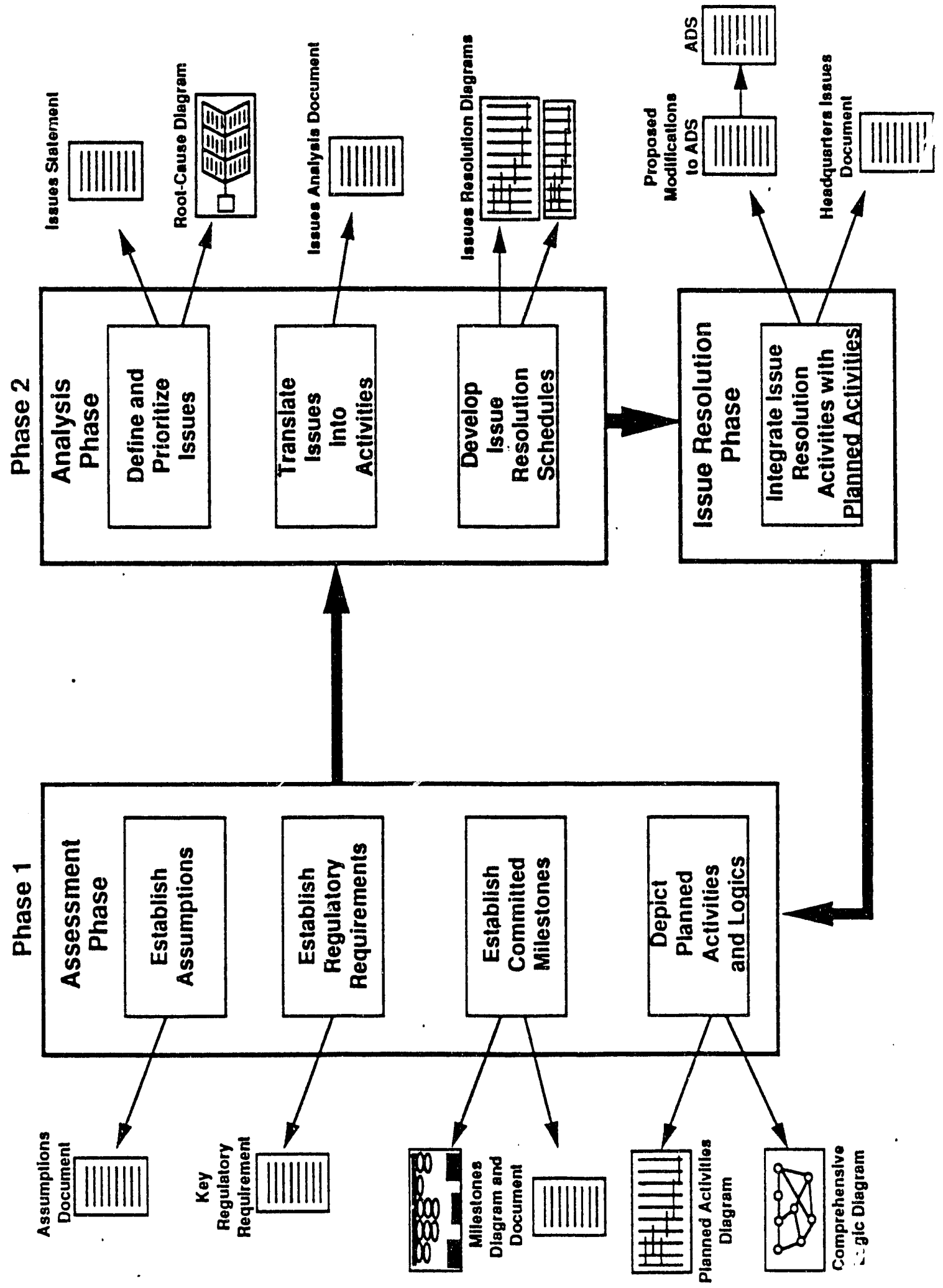


Chapter 4.0, Milestones, contains the milestones associated with SST activities. These milestones are based upon the Tri-Party Agreement and the FY 1993-1997 Five-Year Plan ADSs (Ref. 3).

Chapter 5.0, Planned Activities, lists the planned activities scheduled for SSTs based on the FY 1992-1996 Five-Year PIan ADSs (Ref. 4) and existing program plans.

Chapter 6.0, Logic Diagram, contains the logic diagram that shows the sequence of steps necessary to achieve ultimate closure of the SSTS.

Chapter 7.0, Issues Analysis, includes the issues identified using the information from the assessment phase and from a senior-level inter-

disciplinary review team. Issues Analysis also includes the identification of the activities required to resolve these issues.

Chapter 8.0, Issues Resolution and Desired Activities, presents the issues resolution schedule and the desired activities schedule. The desired activities schedule shows the planned activities schedule modified to show the new and modified activities.

Chapter 9.0, Headquarters Issues, presents those issues that require some degree of DOE-HQ action for resolution. These issues cannot be resolved by Hanford Site activities alone.

Chapter 10.0, References, lists the references used in this document. 
This page intentionally left blank. 


\subsection{ASSUMPTIONS}

The following catalogs the major assumptions used to develop the SST Roadmap. The Roadmap includes all activities associated with the SSTs, their contents, and SST farm contaminated soils and contaminated equipment.

The assumptions listed are part of the planning basis for the SST closure, scheduled to be complete in 2018 as required by the Tri-Party Agreement.

The assumptions do not include contingency for uncertainties in the mission schedule, technical, or cost estimate baselines. Mission uncertainties that could not be quantified and converted into assumptions are identified in the last section of this chapter.

In general, the Roadmap assumes a constant, success-oriented environment; that any changes result in improved execution or facilitation of the planned activities; and that past cleanup commitments and agreements are honored or renegotiated when justified.

\section{Institutional Environment}

Assumptions in this category address continuity in government agencies and other organizations involved in the SST mission. Policies, regulations, and goals of these institutions continue to evolve rapidly.

\section{A. Federal Government}

1. The U.S. Nuclear Regulatory Commission (NRC) is required to license disposal of high-level waste wherever it is disposed. High-level waste when disposed will have to meet release and dose limits specified in 40 Code of Federal Regulations (CFR) 191 (when issued).

2. The roles of other Federal bodies that influence the SST mission (including Congress, the Office of Management and Budget, and the U.S. Environmental Protection Agency [EPA]) are assumed to vary through SST closure. The EPA will continue to expand the state's authority to implement the Federal RCRA program. Future roadmap updates will account for new requirements as necessary. 
B. U.S. Department of Energy (DOE)

1. Policies

a. Determination of land use policy and groundwater remediation policy will influence final site and SST cleanup criteria. Future roadmap updates will account for new policy as necessary.

2. Organization Structure

a. Organizational changes that occur will be accommodated as part of the normal planning process.

C. Mission Office Programs

None identified.

D. State

1. The State will continue to have regulatory authority under Resource Conservation and Recovery Act of 1976 (RCRA) for SST closure.

E. Third Parties

1. Public involvement in planned SST activities (e.g., National Environmental Policy Act [NEPA]) will take place; appropriate time allowances for input/review, including reviews by outside organizations like the National Academy of Sciences, have been incorporated into the schedule baseline.

\section{Regulatory Compliance Environment}

Assumptions in this category address conditions in the current regulatory environment. Policies, regulations, and implementation continue to evolve rapidly.

A. Overall Regulatory Compliance Environment

1. The Tri-Party Agreement will continue to govern site cleạnup activities; changes to milestones will be made and reflected in future roadmap updates according to Tri-Party Agreement protocol when warranted. 
2. Relief from current regulatory requirements will be undertaken through petitions for rulemaking changes, or inconsistency determinations, per the AEA of 1954, or through compliance schedules established in the Tri-Party Agreement.

\section{B. Federal Regulations}

1. The DOE process implementing NEPA will be followed for all planned activities. The Closure SEIS process is expected to take at least 5.5 years from the notice of intent to the record of decision. The tank safety environmental impact statement (EIS) process from the notice of intent to the record of decision is expected to take 2.5 years.

2. The Technology Demonstrations will require NEPA documentation.

3. Reauthorization of RCRA will not significantly affect work scope or schedule.

\section{State Regulations}

1. In order to. meet milestone dates, the RCRA Part B permit applications for new waste pretreatment facilities will be submitted and reviewed as the closure options for SSTs are determined.

2. The SST Closure SEIS will address reasonable alternatives for closure and disposal of SST tank waste.

3. The SST closure plan will be finalized, publicly reviewed, and approved by the State before closure of the SSTs.

4. Wastestreams containing listed hazardous waste constituents will be treated to meet applicable regulations governing the disposal of dangerous waste.

5. Currently stored SST waste is not land disposal restricted. Any waste generated during closure will be land disposal restricted and will be treated in accordance with the land disposal restrictions or a variance granted under Title 40 CFR Part 268.

6. Washington Administrative Code (WAC) Dangerous Waste Regulations apply to SST activities; however, where appropriate, technically based regulatory relief will be pursued to allow decision making to be based upon consideration of health-based thresholds, risk-based performance assessment, good practices equivalency, or benefit arguments will be pursued. 
7. Land use planning, including planning to determine final cleanup standards, will be a part of the approved closure plan.

D. Third Party Involvement

1. Public interaction will be required throughout the waste characterization, closure, and RCRA permitting process as well as the NEPA frocess.

\section{E. New Requirement Discovery}

1. Internal reviews, and reviews by outside organizations, such as Tiger Teams, Technical Safety Appraisal, Advisory Committee on Nuclear Facility Safety, General Accounting Office, Defense Nuclear Facilities Safety Board, the National Academy of Sciences, and the High-Level Radioactive Waste Tanks Task Force, will identify additional changes, or new safety issues, that require significant work scope or schedule alterations.

\section{Project Management}

Assumptions in this section address the project management activities and the technical, schedule, and cost baselines of the SST mission.

A. Resources

1. Funding, equipment, facilities, personnel, technical skills, and the supporting infrastructure needed to complete the SST mission will be available when required.

\section{B. Budget Process}

1. The "required" funding level identified in the Five-Year Plan ADSs, which covers the activities described in this Roadmap for FY 1993 to FY 1997, will be provided to complete SST closure by 2018 , and to complete all activities by about 2040. The Roadmap assumes FY 1991 funding at the authorization level and FY 1992 funding at the guidance level.

2. Short term schedule delays, caused by receiving "guidance" funding which is less than the "required" funding, are assumed to be recoverable early in the mission by replanning and increasing later year "required" funding. Protracted (multi year) delays will result in an inability to successfully meet milestones. 
3. Environmental Restoration and Remedial Action (ERRA) funding will continue to be in the "expense" category.

\section{Project Baselines}

1. Cost and Schedule

a. The Five-Year Plan cost estimates will be confirmed by independent cost assessments.

\section{Technical}

a. Waste characterization for current operations, closure, and other key phases of SST mission, will be in accordance with the SST Waste Characterization Plan.

b. The Waste Characterization Plan for the Hanford Site SingleShell Tanks (Ref. 5) will be approved by the State.

c. Laboratory capability will be acquired to support the SST mission.

d. The availability and timing of the high-level waste repository(s) and the Waste Iso?ation Pilot Plant will infiuence SST closure; interim storage alternatives for onsite storage of packaged waste wi?l be used if needed.

e. Existing permitted facilities will be used for mission continuity until replaced by new, regulation-compliant facilities.

f. Upgrades or alterations to existing facilities that support SST waste treatment will significantly affect SST closure.

g. Waste treatment will be required for SST waste. New or modified waste forms will be required for final disposal of high-level and low-level waste.

h. The SST will close under appropriate regulations. A closure plan and, as necessary, a postclosure permit application will be the approach used.

\section{External Support}

1. None identified. 
E. Technical Support

1. Current planining has identified the available technology options. Technology and engineering data to support NEPA and closure planning will be developed from a research development, demonstration, testing, and evaluation process.

2. The Technology Development Program will integrate selected Hanford Site SST technology needs and planning into the national program. The Technology Development Program is in transition from specifically funded technology to integrated demonstrations. The Environmental Restoration Program and Waste Management Program will continue to identify and seek funding for technology needs not clearly funded via the technical development integrated demonstration program. 


\subsection{REGULATORY REQUIREMENTS}

The following presents the regulatory requirements for which the Hanford Site must adhere to for SST operation, closure, and waste disposal.

Requirements are Federal, State, and local regulations, codes, and agreements with which the DOE. is responsible to comply.

Regulatory requirements for SSTs are broken down into three categories; operating requirements, closure-related requirements, and waste disposalrelated requirements.

\section{Operating Requirements}

1. RCRA: General treatment, storage, and disposal (TSD) operating

2. RCRA: Integrity assessments and retrofits

3. Response to leaking tanks

4. RCRA: Ignitable, reactive, and incompatible wastes

5. Waste analysis and characterization

6. State performance standards for TSDS

7. Substantive DOE Orders affecting radioactive and hazardous waste management

8. Radiation protection

9. Air emissions and discharges to soits

10. Environment, safety and health appraisals

11. Public Law 101-510, Section 3137, "Safety Measures for Waste Tanks at Hanford Nuclear Reservation"

\section{Closure-Related Requirements}

11. RCRA Closure plan.

12. RCRA closure performance standards

13. RCRA and Tri-Party Agreement tank closure requirements

14. Treatment and interim storage of retrieved waste 
15. In situ disposal requirements

16. RCRA corrective action

17. Tri-Party Agreement closure-related milestones

18. Environmental Review (NEPA, SEPA)

19. Comprehensive Environmental Response Compensation Liability Act of 1980 (CERCLA) remediation

\section{Waste Disposal-Related Requirements}

20. Disposal of retrieved wastes and treatment residuals 
1. REGULATION: Basic RCRA operating requirements for TSD tanks (WAC 173-303-280 to 390; 40 CFR 265.194 and 265.195; and Tri-Party Agreement Milestone M-24-07)

REGULATING AUTHORITY: State of Washington

\section{REQUIREMENTS ARE:}

1. Inspection plans and inspections.

2. Notifications, record keeping, and reporting.

3. Security, preparedness, and prevention.

4. Contingency planning and emergency response.

5. Training,

6. Prevention of spills and overfiliing.

7. Installation of groundwater monitoring wells around the SSTS.

\section{STATUS:}

SST operation generally fulfills these requirements; compliance is assessed on a regular basis and corrective measures are implemented when necessary. 
2. REGULATION: RCRA tank assessment and retrofit requirements

(40 CFR 265.191 to 265.193, WAC 173-303-640)

REGULATORY AUTHORITY: State of Washington

\section{REQUIREMENTS ARE:}

1. Perform integrity assessments and certify tank integrity.

2. Retrofit tanks for leak detection.

3. Retrofit tanks to provide secondary containment.

\section{APPLICABILITY:}

Retrofitting the SSTs to provide secondary containment as specified by RCRA is not feasible, due to radiation safety concerns and the size of and construction of the tanks.

Integrity assessments of the kind specified in RCRA cannot be conducted for the SSTS, due to the nature and condition of the tanks and radiation safety concerns.

\section{STATUS:}

The SSTs are not certified as having integrity sufficient for their current use, and are acknowledged to be unfit for use. The SSTs will not be upgraded to meet secondary containment requirements because they are undergoing closure in accordance with schedule in the Tri-Party Agreement.

SST leak detection capabilities do not meet RCRA requirements; improvements are technically feasible but constrained by limited resources.

Agreements with the Washington State Department of Ecology (Ecology) have been reached to allow use of certain single-contained lines for waste transfers associated with interim stabilization. Some single-contained lines that should have been retrofitted or replaced with double-encased lines are still used for waste transfers associated with interim stabilization. 
3. REGULATION: Near-term waste removal requirements (40 CFR 265.196; TriParty Agreement Milestones M-05-00 and M-07-00)

REGULATING AUTHORITY: U.S. Environmental Protection Agency and the State of Washington

\section{REQUIREMENTS ARE:}

1. Remove sufficient wastes to prevent further releases from leaking tanks as soon as is practicable.

2. Retrofit leaking tanks to provide secondary containment, or close them under RCRA.

3. Interim stabilization of all tanks but $\mathrm{C}-105$ and $\mathrm{C}-106$ by September 1995. Interim stabilization of $\mathrm{C}-105$ and $\mathrm{C}-106$ by September 1996. Begin to retrieve waste from one tank by October 1997.

\section{STATUS:}

Hanford's interim stabilization program has reduced and will continue to reduce the potential for further releases from the SSTs. The schedule to complete interim stabilization has been agreed to by DOE, Ecology, and EPA through the Tri-Party Agreement.

The SSTs will be closed. 
4. REGULATION: RCRA requirements for ignitable, reactive, and incompatible wastes (WAC 173-303-395; 40 CFR 265.198 and 265.199)

REGULATING AUTHORITY: State of Washington

\section{REQUIREMENTS ARE:}

1. Avoid risks of waste ignition or reactions.

2. Keep incompatible wastes separated.

\section{STATUS:}

The SSTs are operated to prevent temperatures from reaching levels that could be expected to cause reactions in stored waste. However, uncertainty concerning waste characteristics, and monitoring deficiencies, prevent certainty that current measures are adequate. Potential reactions involving ferrocyanides, hydrogen, and acetates are under review. 
5. REGULATION: Waste analys is and characterization (40 CFR 265.13, 265.200, DOE Order 5820.2A; Tri-Party Agreement Milestone M-10-00)

REGULATORY AUTHORITY: U.S. Department of Energy, U.S. Environmental Protection Agency, U.S. Nuclear Regulatory Commission, and the State of Washington

\section{REQUIREMENTS ARE:}

1. A detailed chemical and physical characterization of hazardous wastes must be obtained prior to TSD. TSD operators must have a written waste analysis plan.

2. High-level waste in single contained systems must be characterized consistent with radiation protection requirements and safa storage needs, and monitored to provide liquid volume data and waste inventory data.

3. Low-level waste characterization must be adequate to permit proper segregation, TSD, and include information on the physical and chemical characteristics of the wastes and major radionuclides and their concentrations.

4. DOE must complete analysis of at least two core samples from each SST by 1998, conforming to sampling and analysis plans approved by the State. Intermediate milestones associated with this sampling and analysis effort are also specified in the Tri-Party Agreement.

5. DOE must provide characterization of radioactive and hazardous constituents in SSTs in support of technology per the Hanford Defense Waste (HDW)-EIS (Ref. 6) record of decision.

6. Characterization to support NRC designation as high-level waste or incidental waste.

\section{APPLICABILITY:}

Waste analysis requirements that involve activities that do not produce benefits sufficient to justify their dose and costs may be applicable.

\section{STATUS:}

Historical records and process knowledge are sufficient to support continued storage of SST waste, and/or safe implementation of interim stabilization and isolation alternatives. This knowledge is not sufficient to provide a "detailed chemical and physical characterization" of SST wastes.

Waste transferred to double-shell tanks during interim stabilization are analyzed in accordance with a written waste analysis plar. 
6. REGULATION: State performance standards for TSDS (WAC 173-303-283)

REGULATING AUTHORITY: State of Washington

\section{REQUIREMENTS ARE:}

The owner/operator must design, construct, operate, and maintain a dangerous waste facility to the maximum extent technically possible to:

1. Prevent degradation of groundwater quality.

2. Prevent degradation of air quality.

3. Prevent degradation of surface water quality.

4. Prevent impairment of flora and fauna.

5. Prevent endangerment of health of employees or public near the facility.

\section{APPLICABILITY:}

These state regulatory provisions are applicable to DOE as operator of the SSTs. Because the SSTs cannot meet all interim status standards they are closed in the compliance schedule set forth in the Tri-Party Agreement. 
7. REGULATION: Substantive DOE Orders affecting hazardous waste management (DOE Orders $5400.1,5480.1,5480.4$, others)

REGULATORY AUTHORITY: U.S. Department of Energy

\section{REQUIREMENTS ARE:}

1. Comply with applicable regulations.

2. Protect human health and the environment.

3. Control pollutants to prevent releases.

4. Develop and implement environmental protection plans.

5. Monitor facilities and the environment.

6. Additional requirements in DOE Order 5480.4 .

\section{APPLICABILITY:}

DOE Order 5480.4 establishes requirements for facility design, construction, modification, decommissioning activities, and waste management activities.

DOE Order 5480.1 establishes requirements for environmental protection programs and environmental monitoring requirements.

\section{STATUS:}

The requirements establisined in DOE Orders 5480.1, 5480.4, and 5400.1 are compiled with to the extent consistent with the Atomic Energy Act and the extent to which they are legally applicable. Some RCRA regulations may not apply when RCRA is inconsistent with the Atomic Energy Act. 
8. REGULATION: Radiation protection (40 CFR 61 Subpart H; DOE Orders 5480.1, 5480.1B, 5480.11A, 5820.2A; DOE-RL Order 5480.11A; EPA Radiation Protection Guidance; State programs for sources of airborne radionuclides, WAC 173-480)

REGULATORY AUTHORITY: U.S. Department of Energy, U.S. Environmental Protection Agency, and the State of Washington

\section{REQUIREMENTS ARE:}

1. Maintain offsite dose equivalent from radioactive waste management and emissions to air within specified limits, and as low as reasonably achievable (ALARA).

2. Meet offsite limits at the point of release for actively ventilated SSTs.

3. Meet specified radiation limits at release points.

4. Maintain occupational dose equivalents within specified limits, and ALARA.

5. Employ best available radiation control technology to limit releases of radiation to the air.

6. For high-level waste in single-contained systems, provide methods for identification of failed containment, and a method for testing system integrity.

\section{STATUS:}

Hanford is consistently below onsite and offsite radiation dose equivalent limits and release limits with a large margin of safety.

SST monitoring is not adequate because no method is provided for testing system integrity.

SST monitoring meet the requirements of DOE Orders and EPA regulations governing radiation protection to the extent practicable and mandated by the AEA. 
9. REGULATION: Air emissions and soil column discha:; (nonradioactive) (WAC 173-400, 173-480, 173-403, and 173-216)

REGULATING AUTHORITY: State of Washington

\section{REQUIREMENTS ARE:}

1. Secure a permit for waste discharges to the soil column.

2. Treat wastes discharged to soil using all known, available, and reasonable technology.

3. Register air emissions sources and maintain emissions within specified 1 imits.

\section{APPLICABILITY:}

Air emission provisions apply statewide. The State waste discharge permit program does not apply to National Pollutant Discharge Elimination System (NPDES) permitted discharges or to practices (e.g., releases from leaking hazardous waste tanks) that are regulated under RCRA. At' present, the SSTs do not discharge any effiuents that would potentially be subject to this program.

\section{STATUS:}

The Hanford Site has not applied for or received any WAC 173-216 permits.

SST effluents that could be generated in the future could be subject to WAC 173-216. Project 1049 (200 Area treated effluent disposal facility) will provide a limited monitoring and diversion capability for these streams, beginning in 1995 . 
10. REGULATION: Environment, Safety, and Health Appraisals (DOE

Order 5482.1B, DOE-RL Order 5482.1B; PL 101-570 Defense Authorization Act of FY 1991)

REGULATING AUTHORITY: U.S. Department of Energy

\section{REQUIREMENTS ARE:}

1. Management appraisals, technical safety appraisals, and environmental surveys and audits must be conducted on a regular basis.

2. Ongoing operations must document risks.

3. Hazards must be eliminated, controlled or mitigated to the extent feasible using reasonable measures.

4. Review the potential for release of high-level waste due to uncontrolled increases in temperature and pressure, by early 1991.

5. Establish a program to research reducing environmental hazards from defense waste and environmental restoration of inactive defense sites.

\section{STATUS:}

The Hanford Site has a program to achieve compliance with environmental, health, and safety analysis requirements in DOE Orders on a continuous basis. The review required by the Defense Authorization Act is underway. 
11. REGULATION: RCRA Closure P1an (40 CFR 265.112, 265.197(c), and 265.310; Tri-Party Agreement Milestone M-09-02, WAC 173-303-610)

REGULATORY AUTHORITY: U.S. Department of Energy, U.S. Environmental Protection Agency, and the State of Washington

\section{REQUIREMENTS ARE:}

1. Prepare a closure plan for all operating RCRA TSD units.

2. Address both clean closure and closure as a landfill in the plan.

3. Submit the closure plan shortly after the last volume of waste is received (SSTs are no longer receiving waste).

4. Submit the closure plan by December 2003 .

\section{APPLICABILITY:}

Tri-Party Agreement Milestone M-09-02 provides for submission of a closure plan in December 2003.

\section{STATUS:}

No closure plan for the SSTs has been prepared or submitted to the State. A closure planning document has been submitted to the State for review and comment. 
12. REGULATION: RCRA closure performance standard (40 CFR 265.111)

REGULATING AUTHORITY: State of Washington

\section{REQUIREMENTS ARE:}

1. Close so as to minimize maintenance requirements.

2. Close so as to control, minimize, or eliminate releases that could threaten human health or the environment.

\section{COMMENTS:}

SST closure will meet these performance standards. 
13. REGULATION: RCRA and Tri-Party Agreement tank closure requirements (40 CFR 265.197; Tri-Party Agreement Action Plan, Section 6.3.1)

REGULATING AUTHORITY: State of Washington

\section{REQUIREMENTS ARE:}

1. Remove or decontaminate all wastes, residues, and contaminated containment system components.

2. Remove or decontaminate soils, unless this is impracticable.

3. If some contaminated soils remain, cap the facility, install groundwater monitoring, and provide postclosure care.

4. For clean closure, provide documentation that groundwater and soils have not been adversely impacted, "as described in WAC 173-303-645."

5. Address the radioactive component of the waste as part of the RCRA closure.

\section{APPLICABILITY:}

Removal and decontamination requirements are not applicable if RCRA requirements are inconsistent with AEA requirements. (The AEA will preempt the RCRA requirements).

WAC 173-303-645 "describes" clean closure only by reference to state regulations that are not applicable to the interim status SSTs. However, as discussed in the Tri-Party Agreement all interim status units will be closed in accordance with the standards of final status. (In situ vitrification might qualify as clean closure under interim status standards, but would not qual ify under the regulations referenced in WAC 173-303-645.)

Source, by-product, and special nuclear materials are regulated under CERCLA but not RCRA. Whether DOE's agreement to address radionuclides during RCRA closure makes such action mandatory is unclear. 
14. REGULATION: Requirements affecting treatment and interim storage of retrieved wastes (RCRA, 42 USC 6901; State dangerous waste regulations, WAC 173-303; the AEA, 42 USC 2100; and DOE Orders)

REGULATORY AUTHORITY: U.S. Department of Energy, U.S. Environmental Protection Agency, and the U.S. Nuclear Regulatory Commission

\section{REQUIREMENTS ARE:}

1. Comply with DOE Orders.

2. Comply with EPA radiation protection standards and guidance.

3. Secure necessary RCRA permits. Comply with permit conditions and RCRA regulations on TSD operation and storage of 1 and disposal restricted wastes.

4. Obtain NRC license for any facilities expressly authorized for long term storage or disposal of high-level waste.

\section{STATUS:}

SST wastes retrieved in the past (and waste being retrieved for interim stabilization) were transferred to other Hanford Site facilities. These facilities are not expected to be adequate for wastes that may be retrieved in the future. DOE is evaluating management alternatives for these wastes. 
15. REGULATION: Requirements affecting in situ disposal (RCRA; State dangerous waste regulations; the AEA and DOE Orders)

REGULATORY AUTHORITY: U.S. Department of Energy, U.S. Environmental Protection Agency, and the State of Washington

\section{REQUIREMENTS ARE:}

1. Retrieve or decontaminate all wastes, residues, and tanks; or secure State approval to reclassify the SSTS as a landfill or land treatment facility prior to undertaking an in situ disposal option. obtain appropriate permits.

2. Retrieve wastes as necessary to assure that the final waste form is not transuranic (TRU), or secure a waiver from DOE Orders.

3. Retrieve wastes as necessary to assure the final waste form is not classified as high-level waste by the NRC, or secure an NRC license, or set NRC concurrence that licensing is not required.

4. Delist the final waste form, or provide a cover, groundwater monitoring, and postclosure care, if the final waste form is regulated as a dangerous waste.

5. Secure necessary air and effluent discharge permits and comply with their terms.

6. Comply with radiation protection requirements during and after in situ disposal.

7. Conduct a performance assessment to shown that the requirements of 40 CFR 191 (or 193) and DOE Order 5820.2A are met. 
16. REGULATION: RCRA Corrective Action (40 CFR 265 Subpart $G$ and 265.197; RCRA Sections $3008(h)$ and $3004(u)$.

REGULATORY AUTHORITY: U.S. Environmental Protection Agency and the State of Washington

\section{REQUIREMENTS ARE:}

1. If any soil or groundwater contamination remains after closure, undertake corrective action as specified in the controling plan, order, or permit.

2. Ancillary equipment to the SSTs, such as catch tank, diversion boxes, transfer piping, and contaminated soils are expected to be addressed under Section $3004(u)$.

\section{STATUS:}

1. Corrective action orders under Section $3008(h)$ of RCRA for interim status units have not been issued.

2. The SST ancillary equipment is currently planned to be closed in accordance with Section $3004(u)$ of RCRA as past-practice units. This may change if the schedule for development of the SST Closure SEIS is accelerated. 
17. REGULATION: Final closure timing and milestones (40 CFR 265.113; TriParty Agreement Milestones M-08-00 and M-09-00)

REGULATORY AUTHORITY: U.S. Department of Energy, U.S. Environmental Protection Agency, and the State of Washington

\section{REQUIREMENTS ARE:}

1. Complete closure within 90-days of approval of a closure plan, untess a longer period of time is approved.

2. Initiate a full-scale tank farm closure demonstration by June 2004 .

3. Complete closure of all tanks b.y June 2018. 
18. REGULATION: Environmental review (NEPA, State Environmental Policy Act [SEPA]; 40 CFR 1500-1508; DOE Order 5440.1C; DOE-RL Order 5440.1A, and related directives)

REGULATING AUTHORITY: U.S. Department of Energy, U.S. Environmenta Protection Agency, and the State of Washington

\section{REQUIREMENTS ARE:}

1. Complete required environmental reviews prior to making affected decisions.

\section{STATUS:}

A risk-based approach is being used to provide a basis for assessment/ disposal alternatives. The EIS and SEIS efforts now planned are expected to fulfill the requirement. 
19. REGULATION: CERCLA (CERCLA Section 120 and 121; 40 CFR Part 300; Executive Order 12580; Tri-Party Agreement)

REGULATING AUTHORITY: U.S. Department of Energy and the U.S. Environmental Protection Agency

\section{REQUIREMENTS ARE:}

1. Conduct a CERCLA remedial investigation/feasibility study for all Federal national priorities list (NPL) sites [CERCLA].

2. Conduct an enhanced RCRA facility investigational corrective measures study for the SSTs and ancillary units rather than a CERCLA RI/FS; address radionuclides [Tri-Party Agreement].

3. Select appropriate remedies, using appropriate procedures and criteria.

4. Implement the selected remedies.

5. Evaluate applicability of RCRA as an applicable or relevant and appropriate requirement.

\section{APPLICABILITY:}

The Tri-Party Agreement specifies that the SSTs be closed as RCRA interim status facilities but subject to final facility standards, rather than under CERCLA. It is the intent that CERCLA requirements be met through the enhanced RCRA RFI/CMS process to be used for some units on the Hanford Site rather than performing both CERCLA RI/FS and RCRA RFI/CMS for the same sites. 
20. REGULATION: Disposal of retrieved waste and treatment residuals (AEA; DOE nrders 5820.2A and others; 40 CFR 191/193; 40 CFR 268;

WAC 173-303-665)

REGI'LATORY AUTHORITY: U.S. Department of Energy, U.S. Environmental

Protection Agency, State of Washington, and the U.S. Nuclear Regulatory Commission

\section{REQUIREMENTS ARE:}

1. High-level waste can be disposed of after stabilization in place provided EPA and NRC standards are met. High-level waste can also be treated so that residues are no longer high-level waste. An NRC license is required for any disposal facility receiving high-level waste.

2. Solidify low-level waste for disposal in a shallow land burial or yreater confinement disposal facility. Meet any conditions that may be imposed by DOE Orders nd meet RCRA requirements if the waste is a) so hazardous.

3. Meet radiation standards established by the EPA and in DOE Orders.

4. Conduct performance assessments to verify disposal facility performance.

5. Prepare and package TRU for shipment to an approved TRU disposal facility.

6. Dispose of hazardous wastes in a RCRA-permitted landfil1. Meet Iand disposal restriction standards, requirements in State regulations, and conditions in the applicable permit.

\section{STATUS:}

There are currently no suitable facilities for the disposal of retrieved and treated SST waste. 


\subsection{MILESTONES}

Table 4-1. Single-Shell Tank Milestones-Milestone Number Order. (sheet 1 of 3 )

\begin{tabular}{|c|c|c|c|c|}
\hline Category & $\begin{array}{c}\text { ADs } \\
\text { number }\end{array}$ & $\begin{array}{l}\text { Milestone } \\
\text { date }\end{array}$ & $\begin{array}{l}\text { Tri-Party } \\
\text { Agreement } \\
\text { mi lestone }\end{array}$ & Milestone description \\
\hline WM & & Annual & $M-04-00$ & Provide annual reports of SST waste treatability studies. \\
\hline WM & & $12 / 89$ & $M-04-01$ & $\begin{array}{l}\text { Provide letter to Ecology describing work scope to be included } \\
\text { in September } 1990 \text { report. }\end{array}$ \\
\hline WM & 9305 & $9 / 95$ & $M-05-00$ & Complete interim stabilization (total 147 out of 149 ). \\
\hline WM & & $9 / 89$ & M-05-01 & Interim stabilize 3 sSTs (total 101 out of 149 ) $\cdots 3$ tanks. \\
\hline \multirow[t]{2}{*}{ WM } & 9305 & $9 / 90$ & M-05-02 & Interim stabilize 4 ssts (total 105 out of 149 ) $\cdots 4$ tanks. \\
\hline & & & $M-05-02$ & Interim stabilize 4 SSTs (total 105 out of 149 ) $\cdots 4$ tanks. \\
\hline \multirow[t]{2}{*}{ mim } & 9305 & $9 / 91$ & $M-05-03$ & Interim stabilize 9 ssTs (total 115 out of 149 ) $\ldots 9$ tanks. \\
\hline & & & $10503 \%$ & Interim stabilize 4 SSTs (total 109 out of 149 ) $\cdots 4$ tanks. \\
\hline \multirow[t]{2}{*}{ WM } & 9305 & $9 / 92$ & $M-05-04$ & Interim stabilize 9 SSTs (total 124 out of 149 ) $\cdots 9$ tanks. \\
\hline & & & $M-05-04$ & Interim stabilize 9 ssis (total 118 out of 149 ) - 9 tanks. \\
\hline \multirow[t]{2}{*}{ 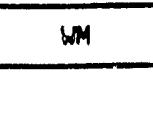 } & 9305 & $9 / 93$ & $M-05-05$ & Interim stabilize 9 ssts (total 133 out of 149$) \therefore 9$ tanks. \\
\hline & & & $10.505 \%$ & Interim stabilize 11 sSTs (total 129 ou: of 149) $\cdots 11$ tanks. \\
\hline \multirow[t]{2}{*}{ WM } & 9305 & $9 / 94$ & $M-05-06$ & Interim stabilize 9 ssts (total 142 out of 149 ) $\cdots 9$ tanks. \\
\hline & & & $100506^{c}$ & Interim stabilize 8 ssts (total 137 out of 149 ) $\cdots 8$ tanks. \\
\hline \multirow[t]{2}{*}{$m$} & 9305 & $9 / 95$ & $M-05-07$ & Interim stabilize 5 sSTs (total 147 out of 149 ) $\ldots 5$ tanks. \\
\hline & & & 10507 & Interim stabilize 10 SSTs (total 147 out of 149 ) $\cdots 10$ tanks. \\
\hline WM & 9305 & $9 / 96$ & M.05.08 & Interim stabilize tanks $241-c-105$ and $241-c-106$. \\
\hline$W M$ & 9305 & $9 / 96$ & $M-05-09$ & Complete interim stabilization and isolation of all 149 SSTs. \\
\hline TO & 5177 & $6 / 94$ & $M-06-00$ & $\begin{array}{l}\text { Develop SST waste retrieval technology and complete scale-model } \\
\text { testing. }\end{array}$ \\
\hline TO & 5177 & $10 / 90$ & $M-06-01$ & $\begin{array}{l}\text { Identify waste retrieval technologies to be tested in a scale } \\
\text { model tank. }\end{array}$ \\
\hline TO & 5177 & $10 / 92$ & $M-06-02$ & Initiate scale-model tank testing. \\
\hline TO & 5177 & $10 / 97$ & $11-07-00$ & $\begin{array}{l}\text { Initiate full-scale demonstration of waste retrieval } \\
\text { technology. }\end{array}$ \\
\hline TO & 5177 & $10 / 93$ & $M-07-01$ & $\begin{array}{l}\text { Submit tank selection criteria, retrieval options, and } \\
\text { recommend selection to Ecology. }\end{array}$ \\
\hline TD & 5177 & $12 / 93$ & $M \cdot 07-02$ & Ecology concur/non-concur of tank selection criteria. \\
\hline
\end{tabular}


Table 4-1. Single-Shell Tank Milestones--Milestone Number Order. (sheet 2 of 3 )

\begin{tabular}{|c|c|c|c|c|}
\hline Category $\mathbf{a}^{\mathbf{a}}$ & $\begin{array}{c}\text { ADS } \\
\text { number }\end{array}$ & $\begin{array}{l}\text { Milestone } \\
\text { date }\end{array}$ & $\begin{array}{l}\text { Tri-party } \\
\text { Agreement } \\
\text { milestone }\end{array}$ & Milestone description \\
\hline TD & 5177 & $12 / 94$ & $M-07-03$ & $\begin{array}{l}\text { Complete design for installation of required waste retrieval } \\
\text { equipment. }\end{array}$ \\
\hline TO & 5177 & $10 / 97$ & $M-07-04$ & $\begin{array}{l}\text { Submit completion date and completion criteria for full-scale } \\
\text { demonstration to Ecology. }\end{array}$ \\
\hline TO & 517 & $12 / 97$ & $M-07-05$ & $\begin{array}{l}\text { Ecology concurrence/non-concurrence of completion date/ } \\
\text { criteria. }\end{array}$ \\
\hline ER & & $6 / 04$ & $M-08-00$ & Initiate full-scale tank farm closure demonstration project. \\
\hline ER & & $1 / 99$ & $M-08-01$ & $\begin{array}{l}\text { Submit tank farm selection criteria, closure method(s), tank } \\
\text { farm selection rationale, and recommended tank farm selection } \\
\text { to Ecology for approval. }\end{array}$ \\
\hline ER & & $1 / 09$ & $M-08-02$ & $\begin{array}{l}\text { Complete final design for the installation of required piping } \\
\text { and other required equipment. }\end{array}$ \\
\hline ER & 5175 & $12 / 03$ & $M-08-03$ & $\begin{array}{l}\text { Submit tank farm closure plan for selected tank farm to Ecology } \\
\text { for approval. }\end{array}$ \\
\hline ER & & $6 / 18$ & $M-09-00$ & Complete elosure of all A49 SSTs. \\
\hline ER & 5175 & $6 / 02$ & $M-09-01$ & $\begin{array}{l}\text { Complete preparation of the supplemental Environmental Impact } \\
\text { statement and issue draft for public review. }\end{array}$ \\
\hline ER & 5175 & $12 / 03$ & $M-09-02$ & Submit closure plan to Ecology for approval. \\
\hline ER & 5176 & $9 / 98$ & $M-10 \cdot 00$ & $\begin{array}{l}\text { Complete analysis of at least two complete core samples from } \\
\text { each of the } 149 \text { SSis. }\end{array}$ \\
\hline ER & 5176 & $3 / 89$ & $M-10-01$ & $\begin{array}{l}\text { Submit draft waste sampling and analys is plan to the National } \\
\text { Academy of Sciences, Ecology, and U.S. Environmental Protection } \\
\text { Agency. }\end{array}$ \\
\hline$E R$ & 5176 & $5 / 89$ & $M-10-02$ & $\begin{array}{l}\text { Submit draft yaste sampling and analys is plan to Ecology for } \\
\text { approval. }\end{array}$ \\
\hline ER & 5176 & $12 / 89$ & $M=10-03$ & Obtain 15 core samples from 2 tanks. \\
\hline ER & 5176 & $12 / 90$ & $M-10-04$ & Obtain 4 core samples from 2 tanks. \\
\hline ER & 5176 & $9 / 91$ & $191004 \mathrm{c}$ & Obtain 4 core samples from 2 tanks. \\
\hline ER & 5176 & $9 / 91$ & $M-10-05$ & Obtain 16 core samples from 8 tanks. \\
\hline ER & 5176 & $9 / 92$ & $M-10-06$ & Obtain 24 core samples from 12 tanks. \\
\hline ER & 5176 & $9 / 93$ & $M-10-07$ & Obtain 24 core samples from 12 tanks. \\
\hline ER & 5176 & $9 / 94$ & $M-10-08$ & Obtain 44 core samples from 22 tanks. \\
\hline ER & 5176 & $9 / 95$ & $M-10-09$ & Obtain 48 core samples from 24 tanks. \\
\hline ER & 5176 & $9 / 96$ & $M-10-10$ & Obtain 48 core samples from 24 tanks. \\
\hline ER & 5176 & $9 / 97$ & $M-10-11$ & Obtain 48 core samples from 24 tanks. \\
\hline
\end{tabular}


Table 4-1. Single-Shell Tank Milestones--Milestone Number Order. (sheet 3 of 3 )

\begin{tabular}{|c|c|c|c|c|}
\hline Category ${ }^{a}$ & $\begin{array}{l}\text { ADS }^{b} \\
\text { number }\end{array}$ & $\begin{array}{c}\text { Milestone } \\
\text { date }\end{array}$ & $\begin{array}{l}\text { Tri-Party } \\
\text { Agreement } \\
\text { milestone }\end{array}$ & Milestone description \\
\hline ER & 5176 & $9 / 98$ & $M-10-12 \cdot$ & Obtain 38 core samples from 19 tanks. \\
\hline WM & 9445 & $6 / 94$ & $14-19-00$ & Complete conceptual design of the hot cell expansion. \\
\hline$M$ & & $6 / 89$ & $M-11-01$ & Complete construction of the environmental hot cell expansion. \\
\hline WM & 9445 & $3 / 92$ & $M-11-02$ & $\begin{array}{l}\text { Complete definitive design of the environmental hot cell } \\
\text { expansion. }\end{array}$ \\
\hline CA & 9307 & $6 / 90$ & $M-23-04$ & Complete waste analysis plans for SSTs. \\
\hline CA & 9307 & $10 / 90$ & $M-23-06$ & Complete contingent plans for SSTs. \\
\hline CA & 9307 & $12 / 90$ & $M-23-11$ & Complete interim status corrective actions for SSTs. \\
\hline WM & 9062 & Annual & 024000 & $\begin{array}{l}\text { Install and monitor groundwater wells around SSTs in compliance } \\
\text { with WAC-173-303-645. }\end{array}$ \\
\hline WM & 9062 & $12 / 91$ & 1.2. & $\begin{array}{l}\text { Install } 3 \text { additional wells around the SSTs for a total of } 15 \\
\text { RCRA monitoring wells. }\end{array}$ \\
\hline WM & 9062 & $12 / 91$ & $4+450$ & Install 18 additional RCRA wells around the SSTS ( 33 total). \\
\hline
\end{tabular}

NOTE:

SST = single-shell tank.

Ecology = Washington State Department of Ecology.

RCRA = Resource Conservation and Recovery Act of 1976.

aWM = waste management

$T D=$ technology development

$E R=$ enviromental restoration

$C A=$ corrective activity.

${ }_{A D S}=$ activity data sheets.

$C_{M-X X-Y Y}$ represents proposed Tri-Party Agreement milestone changes. 
Table 4-2. External Single-Shell Tank Commitments ${ }^{a}$.

\begin{tabular}{|c|c|c|}
\hline Commitment & Source & $\begin{array}{l}\text { Commitment } \\
\text { completion date }\end{array}$ \\
\hline Identification and monitoring of tanks & Wyden $B i 11^{b}$ & Completed \\
\hline $\begin{array}{l}\text { Action plan for excessive temperature } \\
\text { and pressure in tanks }\end{array}$ & Wyden Bill & Completed \\
\hline $\begin{array}{l}\text { Report on actions taken to promote tank } \\
\text { safety }\end{array}$ & Wyden Bill & May 3, 1991 \\
\hline $\begin{array}{l}\text { Westinghouse Hanford reorganization for } \\
\text { tank safety }\end{array}$ & $\begin{array}{l}\text { Watkins Letterc } \\
\text { to DNFSB }\end{array}$ & Completed \\
\hline $\begin{array}{l}\text { Complete Safety Analysis Report for } \\
\text { ferocyanide instrument tree }\end{array}$ & DNFSB & September 1,1991 \\
\hline $\begin{array}{l}\text { Install interim monitoring and alarm } \\
\text { capability in BY tank farm }\end{array}$ & DNFSB & $\begin{array}{l}\text { Estimated } \\
\text { completion FY } 1992\end{array}$ \\
\hline Initiate sampling of ferrocyanide tanks & DNFSB & December 3,1991 \\
\hline $\begin{array}{l}\text { Update Facility Effluent Monitoring } \\
\text { Plan and inventory of emission sources }\end{array}$ & Tiger Teame & November 9, 1991 \\
\hline $\begin{array}{l}\text { Implement Waste Characterization } P 1 \text { an } \\
\text { for single-shell tanks (equivalent to } \\
\text { Tri-Party Agreement Milestone } M-10 \text { ) }\end{array}$ & Tiger Team & September 30,1998 \\
\hline $\begin{array}{l}\text { Finalize development of data quality } \\
\text { objectives for } 51 \text { existing groundwater } \\
\text { monitoring wells at single-shell tanks }\end{array}$ & Tiger Team & May 31, 1991 \\
\hline
\end{tabular}

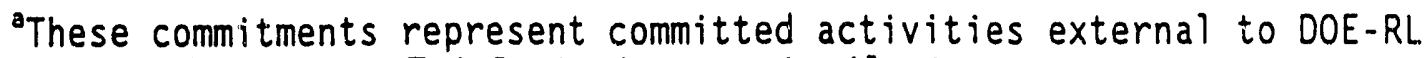
that are in addition to Tri-Party Agreement milestones.

'Publ ic Law 101-510, Section 3137, Safety Measures for Waste Tanks at Hanford Nuclear Reservation, passed November 5, 1990.

'Reference 7 in Chapter 10.0.

DNFSB = Defense Nuclear Facilities Safety Board.

Reference 8 in Chapter 10.0. 


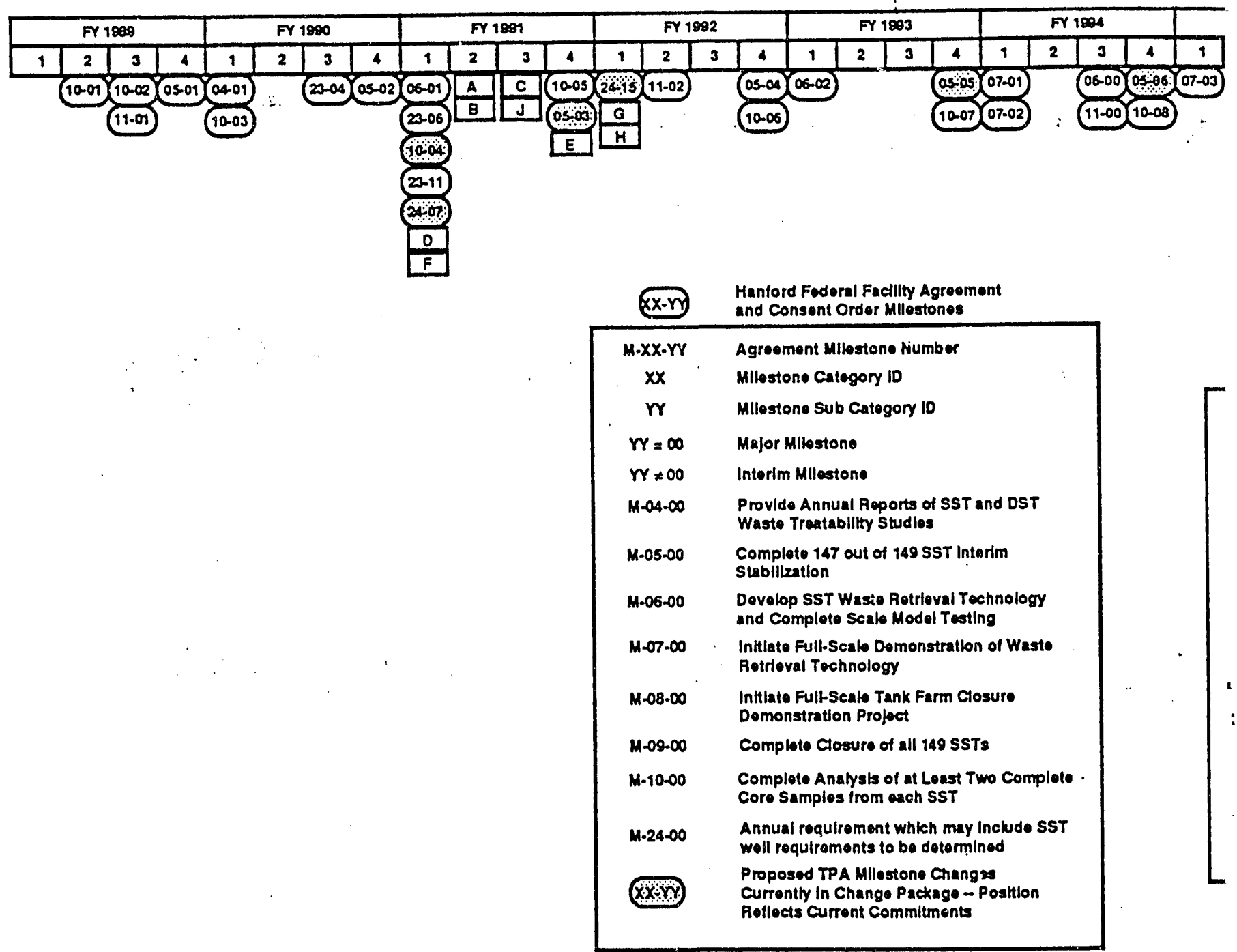


DOE/RL $-90-48$

Predecisional Draft

$4 / 5 / 91$

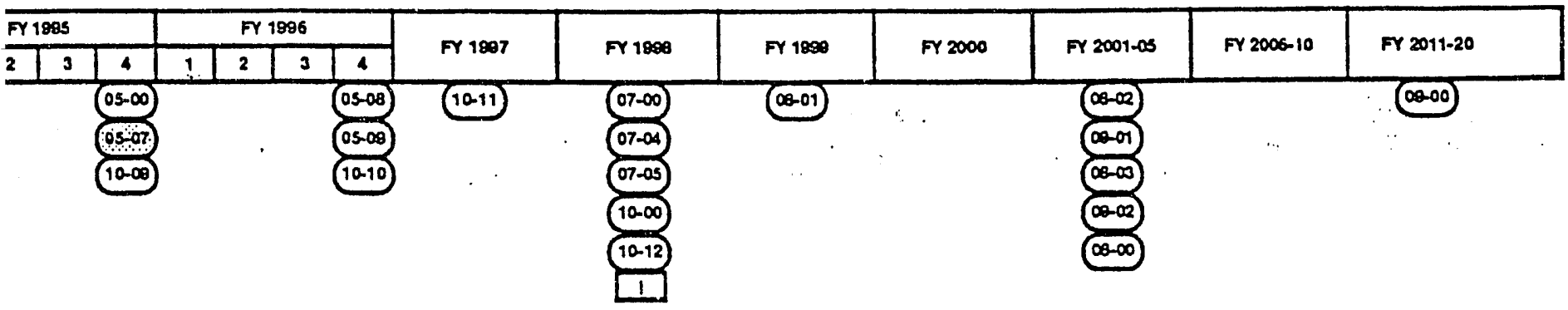

Roport on Actlons Taken to Promote Tank Salory (5/3/91)

WHC Reorgantzation for Tank Safoty (12/1/90)

completed

Complote SAR for Forrcyanido Instrument Troo (2/1/81)

Install Interim Monltoring and Alarm Capablilty in BY

Tank Farm (12/31/90)

estimated

complote FY 92

Inltlate Sampling of Forrocyanlde Tanks (12/3/91)

Tiger Toam: Updute Faclilty Effluent Monitoring Plan and Inventory or EmmistonSources (11/2/91)

Tiger Toam: Implement Waste Chancterization Plan

tor SSTs (equivalent to TPA MS M-10) (9/30/98)

Tigor Toam: Finallze Devolopment of Data Quality Objoctives for 51 Existing Ground Water Monitoring Wolls at SSTs (5/31/91)

Figure 4-1. Milestone Diagram for External Single-Shell Tank Commitments. 
DOE/RL- $90-48$

Predecisional Draft

$4 / 5 / 91$

\subsection{PLANNED ACTIVITIES}


This page intentionally left blank. 
Planned Activities Schedule

\begin{tabular}{|c|c|c|c|c|c|c|c|c|c|c|c|c|c|c|c|c|}
\hline Aetivitie. & sian & Frian Dato & & 19911 & $Y 19921 \mathrm{~F}$ & (1993/F & $\frac{1990 / F Y}{109}$ & $\frac{109311}{1989}$ & 1998 & $\frac{1097}{1097}$ & $\frac{1968}{1998}$ & 1908 & 2000 & 2001 & 2002 & 2003 \\
\hline Intenm sterigen & $10 / 1 / 00$ & $9 / 30 / 96$ & $\frac{19901}{5}$ & 1991 & 1992 & 1993 & & & $\Longrightarrow$ & & & & & & & \\
\hline & & & & & & & & & & & & & & & & \\
\hline Eveduato DOE Requirentants & $10 / 1 / 90$ & $9 / 30 / 96$ & & & & & & & 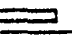 & & & & & & & \\
\hline & & & & & & & & & & & & & & & & \\
\hline Inlerim Stubilzaion of 167 SSTS & $10 / 1100$ & $9 / 30 / 85$ & & & & & & $\Longrightarrow$ & & & & & & & & \\
\hline & & & & & & & & & & & & & & & & \\
\hline Wollo SSTE & 1011180 & $9 / 30 / 96$ & $\equiv$ & & & & & & $\Xi$ & & & & & & & \\
\hline & & & & & & & & & & & & & & & & \\
\hline Stundite 105 and $106 \mathrm{C}$ & $1011 / 94$ & $9 / 30 / 96$ & & & & & & $=$ & $\Longrightarrow$ & & & & & & & \\
\hline Tank Porodies & & & & & & & & & & & & & & & & \\
\hline & $10 / 1180$ & $0 / 30100$ & & & & & & & $\Longrightarrow$ & & & & & & & \\
\hline 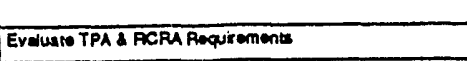 & & & & & & & & & & & & & & & & \\
\hline & 1011190 & $10 / 1 / 90$ & 1 & & & & & & & & & & & & & \\
\hline & $10 / 1 / 90$ & & ㅁ & & & & & & & & & & & & & \\
\hline Dovelop thewo cheractrization Plen & $10 / 1190$ & $10 / 1100$ & & & & & & & & & & & & & & \\
\hline & & & 1 & & & & & & & & & & & & & \\
\hline 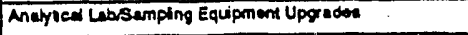 & $10 / 1190$ & $0 / 30 / 94$ & & & & & $\Longrightarrow$ & & & & & & & & & \\
\hline & & & & & & & & & & & & & & & & \\
\hline 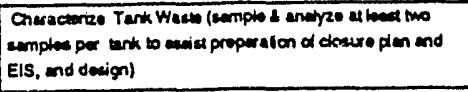 & $10 / 1180$ & $9 / 30 / 08$ & & & & & & & & & & & & & & \\
\hline & & & & & & & & & & & & & & & & \\
\hline Compinimi hevim storeso & $9 / 30 / 96$ & $0 / 30118$ & & & & & & & & & & & & & & \\
\hline & & & & & & & & & & & & & & & & \\
\hline 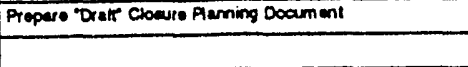 & 1011190 & $10 / 1 / 90$ & 1 & & & & & & & & & & & & & \\
\hline & & & & & & & & & & & & & & & & \\
\hline & 1011190 & $10 / 1 / 98$ & & & & & & & & & D- & & & & & \\
\hline 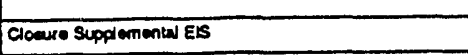 & $10 / 1 / 97$ & $6 / 30 / 4$ & & & & & & & & Е & & & & & & \\
\hline$\div$ & & & & & & & & & & & & & & & & \\
\hline Approved Clowere Plan & $1 / 11103$ & $0 / 30 / 4$ & & & & & & & & & & & & & & \\
\hline & & & & & & & & & & & & & & & & \\
\hline 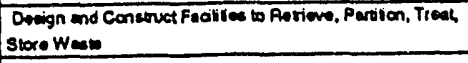 & & & & & & & & & & & & & & & & \\
\hline & & & & & & & & & & & & & & & & \\
\hline 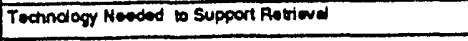 & $10 / 1 / 90$ & $12 / 31 / 97$ & & & & & & & & 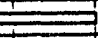 & & & & & & \\
\hline & & & & & & & & & & & & & & & & \\
\hline 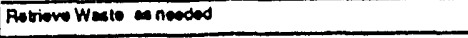 & $1011 / 8$ & $9 / 30 / 17$ & & & & & & & & & & & & & & \\
\hline & & & & & & & & & & & & & & & & \\
\hline Suosicones Provention Dovwlopment & $10 / 1190$ & $9 / 3018$ & & & & & & & & & & & & & & \\
\hline Subsidencos Prevonion & $10011 / 4$ & Tonon & & & & & & & & & & & & & & \\
\hline Barrier and Moriloring Domotopment & $10 / 1 / 90$ & $8 / 30105$ & & & & & & $=$ & & & & & & & & \\
\hline+5 & & & & & & & & 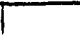 & & & & & & & & \\
\hline
\end{tabular}


DOE/RL- $90-48$

Predecisional Draft

$4 / 5 / 91$

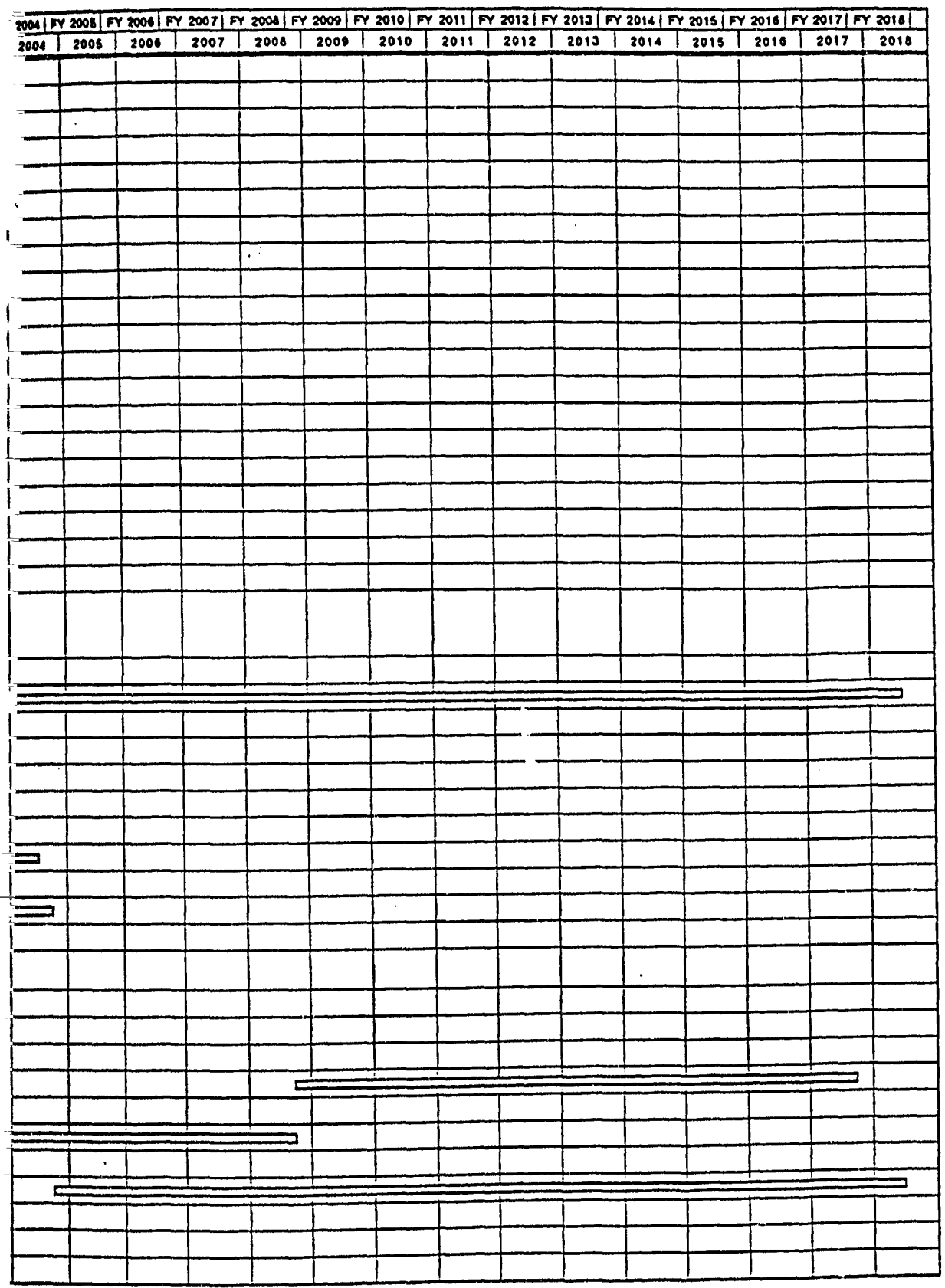




\begin{tabular}{|c|c|c|c|c|c|c|c|c|c|c|c|c|c|c|c|c|}
\hline \multirow{2}{*}{ Aotivities } & \multirow{2}{*}{ stan } & \multirow{2}{*}{ Firien Dans } & \multicolumn{14}{|c|}{ 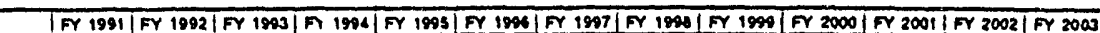 } \\
\hline & & & 1990 & 1991 & 1992 & 1093 & 1904 & 1995 & 1996 & 1997 & T1980 & 1990 & 2000 & 2001 & 2002 & 200. \\
\hline 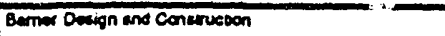 & $10 / 1 / 2$ & $18 / 30 / 18$ & & & & & & & & & & & & & & $=$ \\
\hline & & & & & & & & & & $:$ & & & & & & \\
\hline Fne Clowne & $10 / 1 / 8$ & $0 / 30 / 18$ & & & & & & & & & & & & & & \\
\hline & & & & & & & & & & & & & & & & \\
\hline Denvoponent $\alpha$ Sord Weot Tienster & & & & & & & & & & & & & & & & \\
\hline & & & & & & & & & & & & & & & & \\
\hline 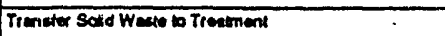 & $\sqrt{6 / 1 / 4}$ & $6 / 30 / 18$ & & & & & & & & & & & & & & \\
\hline & & & & & & & & & & & & & & & & \\
\hline 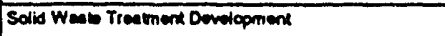 & & & & & & & & & & & & & & & & \\
\hline & & & & & & & & & & & & & & & & \\
\hline Trool Sold Waces & $6 / 1 / 4$ & $8 / 30 / 18$ & & & & & & & & & & & & & & \\
\hline & & & & & & & & & & & & & & & & \\
\hline 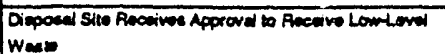 & & & & & & & & & & & & & & & & \\
\hline & & & & & & & & & & & & & & & & \\
\hline 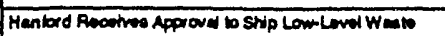 & & & & & & & & & & & & & & & & \\
\hline & & & & & & & & & & & & & & & & \\
\hline 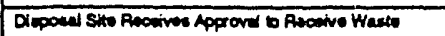 & & & & & & & & & & & & & & & & \\
\hline & & & & & & & & & & & & & & & & \\
\hline 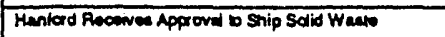 & & & & & & & & & & & & & & & & \\
\hline & & & & & & & & & & & & & & & & \\
\hline 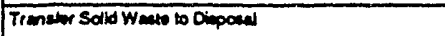 & $10 / 1 / 4$ & $0 / 30 / 18$ & & & & & & & & & & & & & & \\
\hline & & & & & & & & & & & & & & & & \\
\hline Tranetw Tectinology Oow ropopment & & & & & & & & & & & & & & & & \\
\hline & & & & & & & & & & & & & & & & \\
\hline 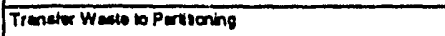 & & & & & & & & & & & & & & & & \\
\hline & & & & & & & & & & & & & & & & \\
\hline Tectnology Nooded lox Perilloning & $10 / 1 / 100$ & $9 / 3016$ & & & & & & & & & & & & & & \\
\hline & & & & & & & & & & & & & & & & \\
\hline Partion Wast: & & & & & & & & & & & & & & & & \\
\hline & & & & & & & & & & & & & & & & \\
\hline 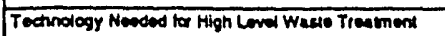 & $10 / 1 / 80$ & $8 / 3016$ & & & & & & & & & & & & & & \\
\hline & & & & & & & & & & & & & & & & \\
\hline 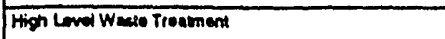 & $10 / 1 / 8$ & $6 / 30 / 18$ & & & & & & & & & & & & & & \\
\hline & & & & & & & & & & & & & & & & \\
\hline Tent Strago operebione & $6 / 114$ & $8 / 30118$ & & & & & & & & & & & & & & \\
\hline & & & & & & & & & & & & & & & & \\
\hline 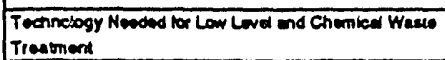 & $10 / 1100$ & $0 / 3018$ & & & & & & & & & & & & & & \\
\hline & & & & & & & & & & & & & & & & \\
\hline Low Lova Wasto Ticarmant & $8 / 114$ & $10 / 30 / 18$ & & & & & & & & & & & & & & \\
\hline & & & & & & & & & & & & 7 & & & & \\
\hline 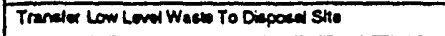 & $0 / 114$ & $8 / 30 / 18$ & & & & & & & & & & & & & & \\
\hline & & & & & & & & & & & & & & & & \\
\hline 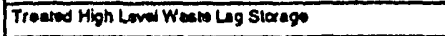 & $6 / 114$ & $10 / 30 / 18$ & & & & & & & & & & & & & & \\
\hline & & & & & & & & & & & & & & & & \\
\hline 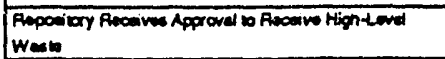 & $111 / 10$ & $111 / 10$ & & & & & & & & & & & & & & \\
\hline
\end{tabular}


DOE/RL- $90-48$

Predecisional Draft

$4 / 5 / 91$

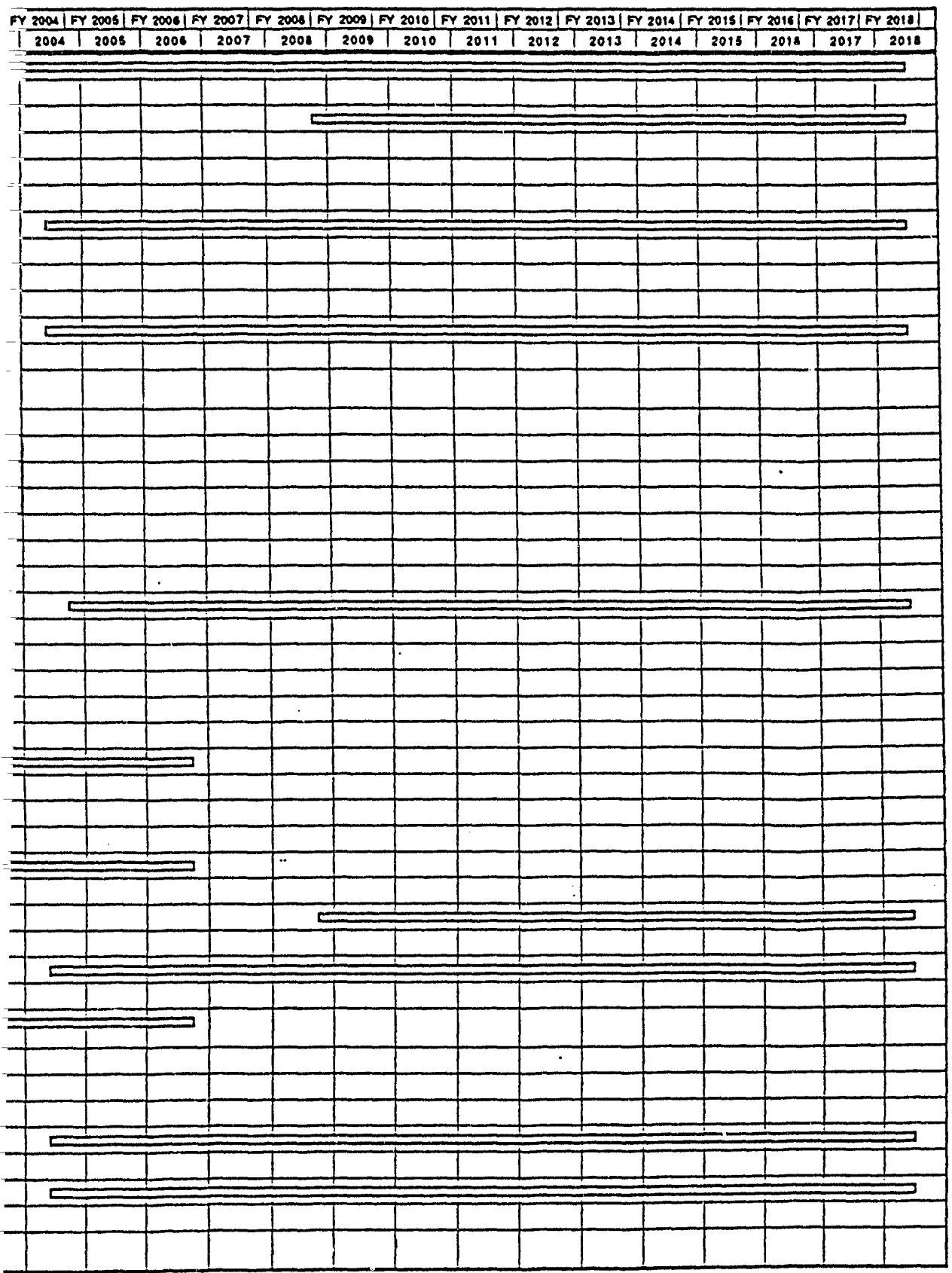


Planned Activities Schedule

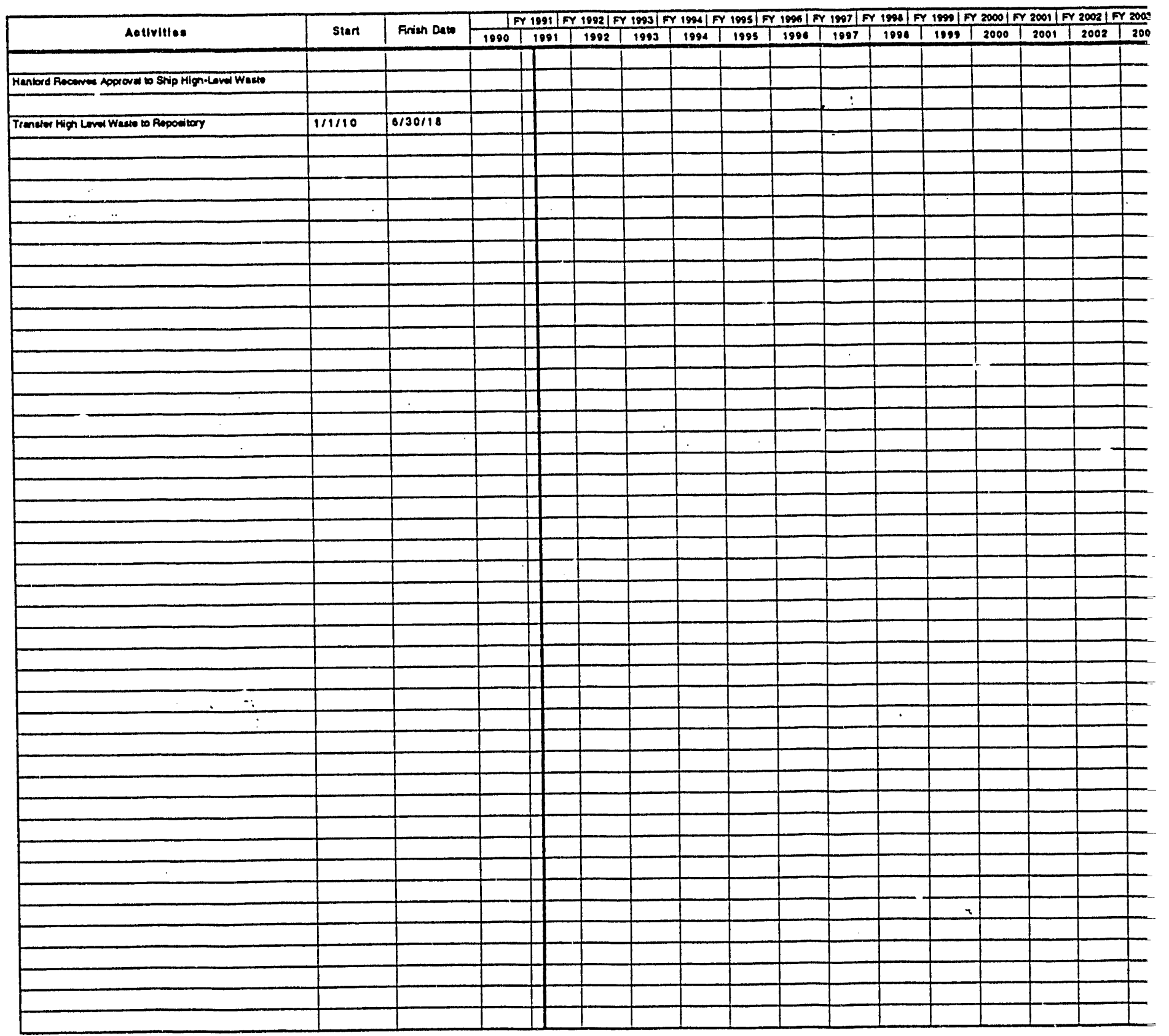




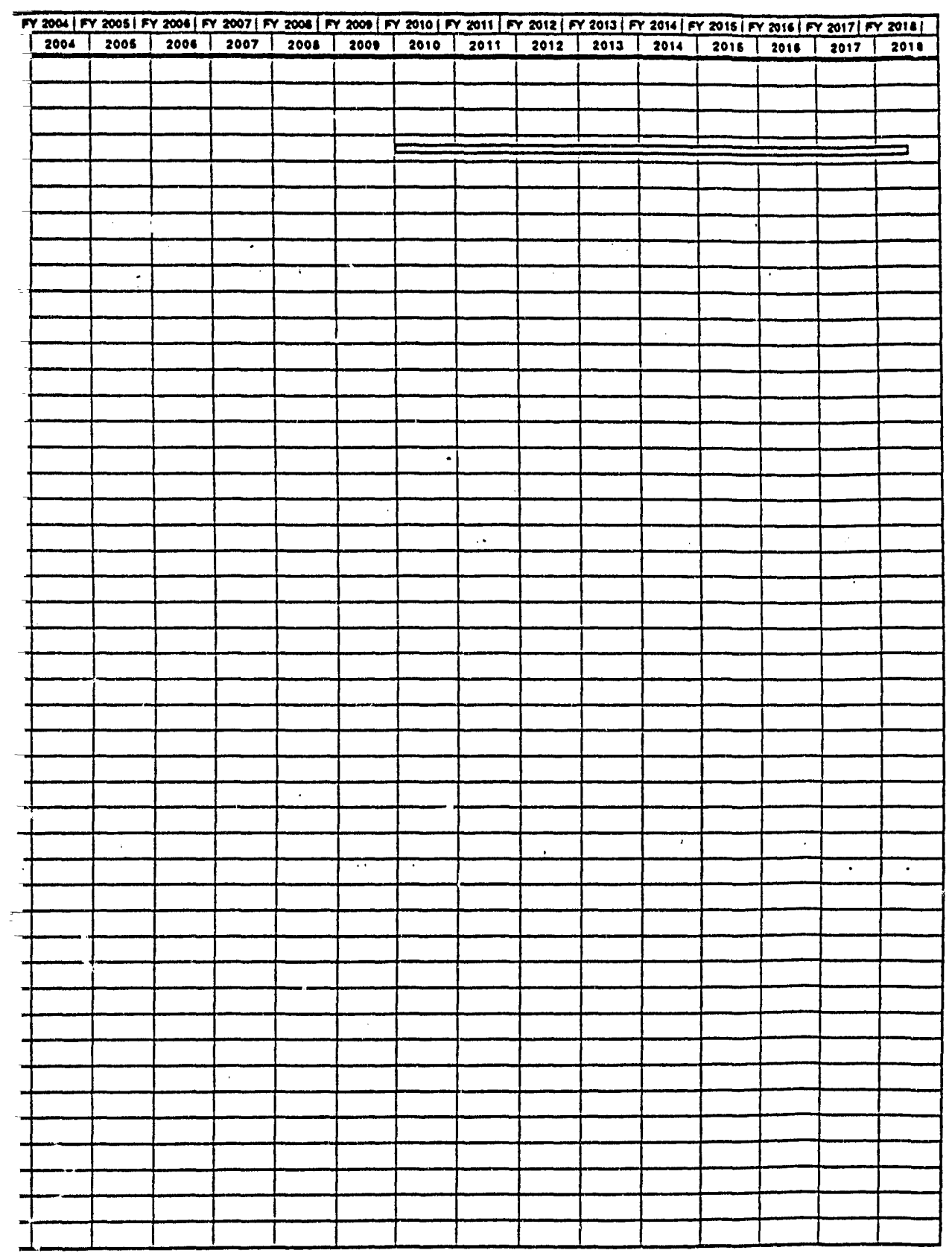




\subsection{LOGIC DIAGRAM}


DOE/RL- $90-48$

Predecisional Draft

$4 / 5 / 91$

This page intentionally left blank. 


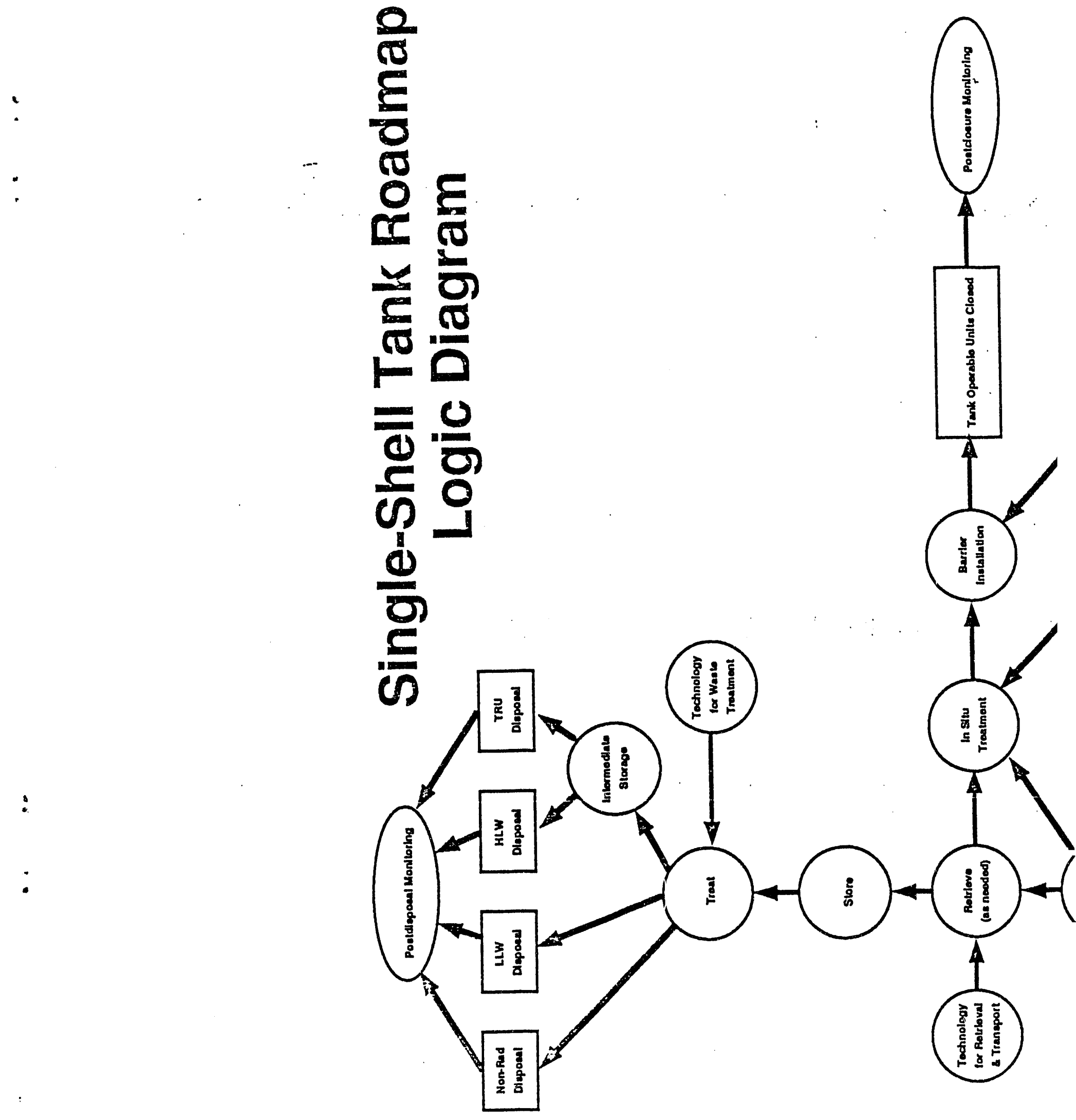




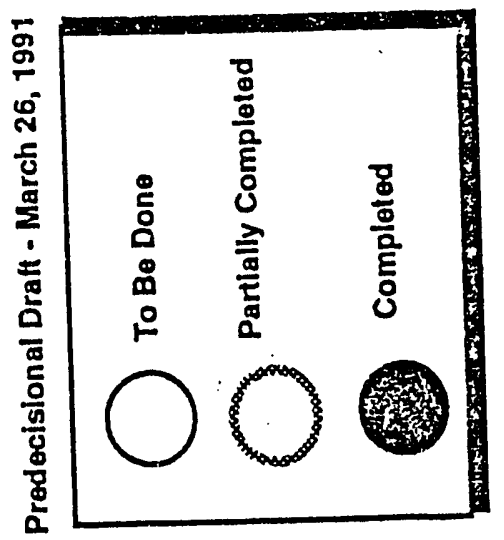

Figure 6-1. Single-Shell Tank Roadmap Logic Diagram.

$$
6-3 / 6-4
$$




\subsection{ISSUES ANALYSIS}


This page intentionally left blank. 
The five primary issues are prioritized as follows:

\begin{tabular}{ll} 
Priority & \multicolumn{1}{c}{ Issue } \\
1 & $\begin{array}{l}\text { Current storage method for waste is not safe or } \\
\text { environmentally sound }\end{array}$ \\
2 & Ability to meet committed closure milestones is uncertain \\
3 & NEPA pathways have not been defined \\
4 & $\begin{array}{l}\text { Regulatory closure requirements (State, EPA, DOE, NRC) do } \\
\text { not agree with HDW-EIS record of decision risk-based } \\
\text { approach } \\
\end{array}$ \\
& Technology development process is still evolving.
\end{tabular}


DOE/RL - $90-48$

Predecisional Draft

This page intentionally left blank. 
DOE/RL $-90-48$

Predecisional Draft

$4 / 5 / 91$

7.1 ISSUE I - CURRENT STORAGE METHOD FOR WASTE IS NOT SAFE OR ENVIRONMENTALLY SOUND 
This page intentionally left blank. 


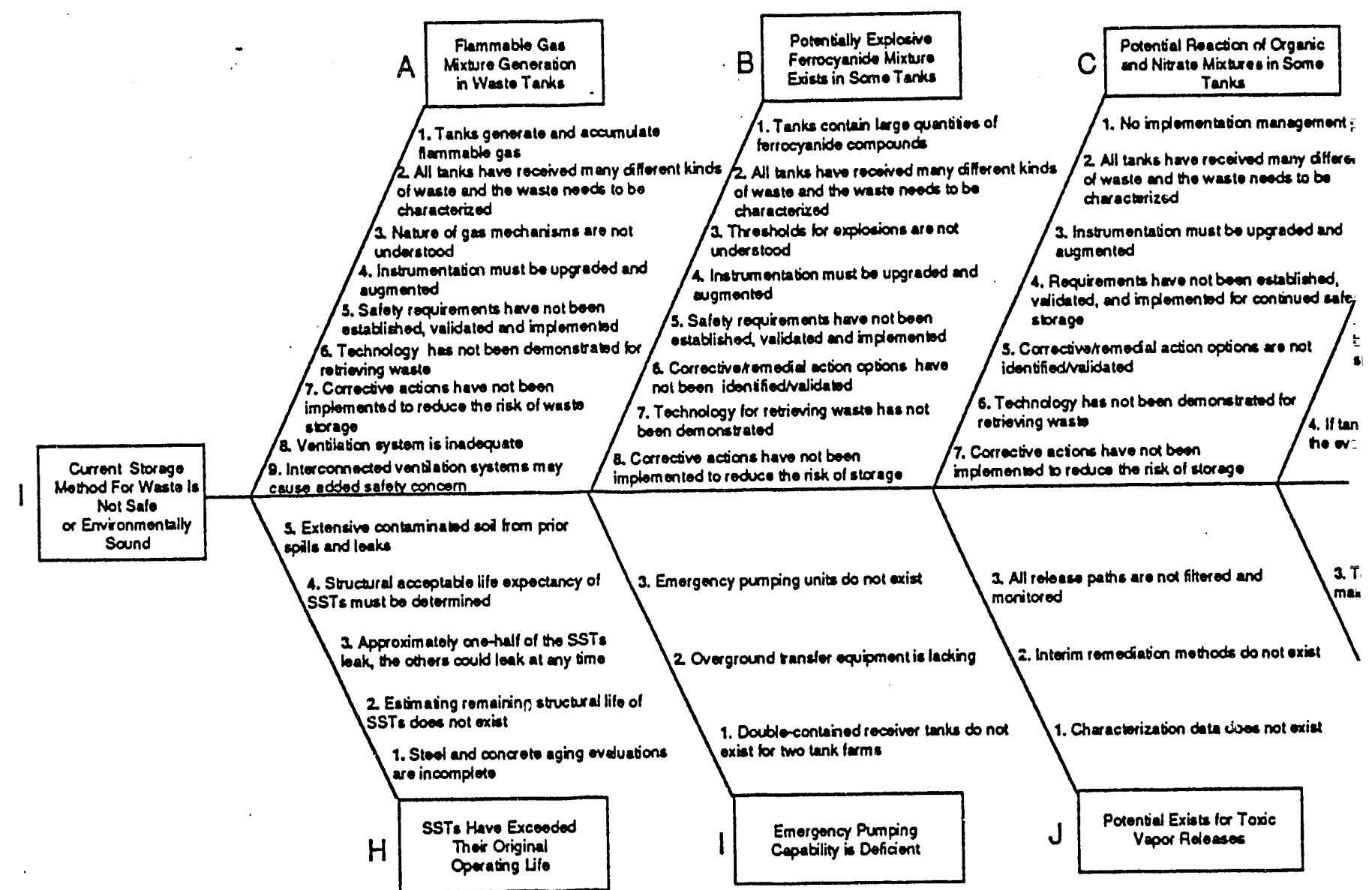


I. Current storage method for waste is not safe or environmentally sound.

A. Flammable gas mixture generation in waste tanks.

1. Tanks generate and accumulate flammable gas.

\section{NEED:}

A plan needs to be prepared to address the problem. Provide support from management to coordinate the work.

\section{ACTIVITY:}

Develop the plan, gain approval and provide management to implement it. Prepare a new or modify existing ADSs. Develop the team with Westinghouse, PNL, other national laboratory, subcontractor, and university staffs.

ACTIONEE: Westinghouse Hanford

ADS: 9123 and 5175 
I. Current storage method for waste is not safe or environmentally sound. A. Flammable gas mixture generation in waste tanks.

2. All tanks have received many different kinds of waste and the waste needs to be characterized.

NEED:

The waste in the different tanks needs to be characterized.

\section{ACTIVITY:}

Core sampling rig modification, sampling methods preparation, core sampling, sample preparation and physical properties determination, chemical analysis, robotics development to permit remute sampling, improved remote characterization and preparing supporting safety assessments and environmental assessments.

ACTIONEE: Westinghouse Hanford

ADS: $5175,5176,9123$; and 9853 
I. Current storage method for waste is not safe or environmentally sound.

A. Flammable gas mixture generation in waste tanks.

3. Nature of gas mechanisms are not understood.

\section{NEED:}

The nature of the gas generation and release mechanisms, both radiolytic and chemical, need to be understood.

ACTIVITY:

Perform gas flow modeling, gas flammability tests, synthetic waste gas generation tests, evaluate data.

ACTIONEE: Westinghouse Hanford

ADS: 9123 
I. Current storage method for waste is not safe or environmentally sound.

A. Flammable gas mixture generation in waste tanks.

4. Instrumentation must be upgraded and augmented.

NEED:

Existing instrumentation needs to be upgraded and additional instrumentation needs to be added to support the monitoring and to provide input to the safety assessments associated with dome gas monitoring and waste characterization.

\section{ACTIVITY:}

Develop dome gas monitoring equipment, TV camera system, gamma scanning in annulus, acoustic monitoring; upgrade instrument tree for temperature and pressure monitoring. Prepare new or modify existing ADSs.

ACTIONEE: Westinghouse Hanford

ADS: 9123 and 9806 
I. Current storage method for waste is not safe or environmentaliy sound.

A. Flammable gas mixture generation in waste tanks.

5. Safety requirements have not been established, validated, and implemented.

\section{NEED:}

Corrective action needs to be taken to reduce the risk for unsafe storage of waste in the tanks. A set of criteria that defines "safe" needs to be establ ished.

\section{ACTIVITY:}

Identify and validate corrective action requirements and concepts; conduct research development demonstration, testing and evaluation as necessary; evaluate alternatives; establish, permit, install, and implement design concepts with necessary reviews and approvals. Prepare new or modify existing ADSs.

ACTIONEE: Westinghouse Hanford

ADS: 9123 and 9806 
I. Current storage method for waste is not safe or environmentally sound.

A. Flammable gas mixture generation in waste tanks.

6. Technology has not been demonstrated for retrieving waste.

NEED:

Establish, validate, and implement requirements for any treatment necessary to allow continued safe storage of the waste until disposal activities are undertaken. Retrieval is one approach that may be applicable to some of the waste.

\section{ACTIVITY:}

Identify and validate retrieval action requirements and concepts; conduct research development demonstration testing and evaluation as necessary; evaluate alternatives; establish, permit, install, and implement design concepts with necessary reviews and approvals. Prepare new or modify existing ADSs.

ACTIONEE: Westinghouse Hanford

ADS: 9123 and 9806 
I. Current storage method for waste is not safe or environmentally sound.

A. Flammable gas mixture generation in waste tanks.

7. Corrective actions have not been implemented to reduce the risk of waste storage.

7

NEED:

A demonstration of waste retrieval technology needs to be performed for those tanks where such retrieval is necessary for treatment and alternative corrective actions (e.g., agitation or dilution) need to be evaluated and implemented.

ACTIVITY:

Develop retrieval technology (both general and Hanford tank specific). Evaluate and implement alternative treatment concepts when validated and appropriate.

ACTIONEE: Westinghouse Hanford

ADS : 5178 


\section{DOE/RL $-90-48$}

Predecisional Draft

I. Current storage method for waste is not safe or environmentally sound.

A. Flammable gas mixture generation in waste tanks.

8. Ventilation system is inadequate.

\section{NEED:}

Appropriate upgrades to the ventilation system need to be defined and implemented.

\section{ACTIVITY:}

Requirements must be defined and the appropriate upgrades designed, approved as required, installed, and operated.

ACTIONEE: Westinghouse Hanford

ADS: 9123,9802 , and 9806 
I. Current storage method for waste is not safe or environmentally sound.

A. Flammable gas mixture generation in waste tanks.

9. Interconnected ventilation systems may cause added safety concern.

\section{NEED:}

Complete studies and implement a system to isolate tanks.

\section{ACTIVITY:}

Studies must be completed to determine if the interconnected ventilation system poses a safety risk that must be corrected. If it does, appropriate design changes must be defined and implemented.

Implement a system to isolate the non-gas generating tanks from the gas generating tanks on a common ventilation system. (It is recognized that the potential fix to this may have wider application.)

ACTIONEE: Westinghouse Hanford

ADS: $9123,9802,9806$, and 9851 
I. Current storage method for waste is not safe or environmentally sound.

B. Potentially explosive ferrocyanide mixture exists in some tanks.

1. Tanks contain large quantities of ferrocyanide compounds.

\section{NEED:}

A plan needs to be prepared to address the potential explosion problem. Provide support from management to coordinate the work. The plan needs to be implemented.

\section{ACTIVITY:}

Develop the plan, gain approval and provide management to implement it. Prepare new or modify existing ADSs. Develop the team with Westinghouse, PNL, other national laboratory, subcontractor, and university staffs.

ACTIONEE: Westinghouse Hanford, DOE-RL, and DOE-HQ

ADS: 5175 and 9850 
I. Current storage method for waste is not safe or environmentally sound.

B. Potentially explosive ferrocyanide mixture exists in some tanks.

2. All tanks have received many different kinds of waste and the waste needs to be characterized.

NEED:

The waste in the different tanks needs to be characterized.

\section{ACTIVITY:}

Core sampling rig modification, sampling methods preparation, core sampling, sample preparation and physical properties determination, chemical analysis, robotics development to permit remote sampling improved remote characterization and preparing supporting safety assessments and environmental assessments.

ACTIONEE: Westinghouse Hanford

ADS: 5175,5176 , and 9850 
I. Current storage method for waste is not safe or environmentaliy sound.

B. Potentially explosive ferrocyanide mixture exists in some tanks.

3. Thresholds for explosives are not understood.

\section{NEED:}

There needs to be a better understanding of the thresholds for explosion of vapors/gases in Hanford Site tanks.

\section{ACTIVITY:}

Perform beaker tests, small- and large-scale explosion tests, and propagation tests; determine minimum reaction temperatures; conduct studies of effects of catalysts and diluent; determine the kinetics of reactions; perform thermal modeling. Prepare new or modify existing ADSs.

ACTIONEE: Westinghouse Hanford and DOE-RL

ADS: 9850 
DOE/RL- $90-48$

I. Current storage method for waste is not safe or environmentally sound.

B. Potentially explosive ferrocyanide mixture exists in some tanks.

4. Instrumentation must be upgraded and augmented.

\section{NEED:}

Existing instrumentation needs to be upgraded and additional instrumentation needs to be added to support the monitoring and to provide input to the safety assessments

\section{ACTIVITY:}

Install continuous temperature monitoring and infrared scanning to detect hot spots; develop and install multifunctional instrument tree.

ACTIONEE: Westinghouse Hanford and DOE-RL

ADS: 9850 and 9806 
I. Current storage method for waste is not safe or environmentally sound.

B. Potentially explosive ferrocyanide mixture exists in some tanks.

5. Safety requirements have not been established, validated, and implemented.

NEED:

Corrective action needs to be taken to reduce the risk for unsafe storage of waste in the tanks. A set of criteria that defines "safe" needs to be established.

\section{ACTIVITY:}

Identify and validate corrective action requirements and concepts; conduct research developmient demonstration, testing and evaluation as necessary; evaluate alternatives; establish, permit, install, and implement design concepts with necessary reviews and approvals. Prepare new or modify existing ADSs.

ACTIONEE: Westinghouse Hanford

ADS: 9123 and 9806 
I. Current storage method for waste is not safe or environmentally sound.

B. Potentially explosive ferrocyanide mixture exists in some tanks.

6. Corrective/remedial actions options have not been identified/validated.

NEED :

Evaluation needs to be completed and corrective action needs to be taken to reduce the risk for safe storage of waste in the tanks.

\section{ACTIVITY:}

Corrective action requirements and concepts must be identified and validated; conduct research development demonstration testing and evaluation as necessary and evaluate alternatives; establish, permit, install, and implement design concepts with necessary reviews and approvals. Prepare new or modify existing ADSs.

ACTIONEE: Westinghouse Hanford, DOE-RL, and DOE-HQ

ADS: 9850 and 9806 
I. Current storage method for waste is not safe or environmentally sound.

B. Potentially explosive ferrocyanide mixture exists in some tanks.

7. Technology for retrieving waste has not been demonstrated.

NEED:

Requirements need to be established, validated, and implemented for any treatment necessary to allow continued safe storage of the waste until disposal activities are undertaken.

\section{ACTIVITY:}

Identify and validate remediation action requirements and concepts; conduct research development demonstration testing and evaluation as necessary; evaluate alternatives; establish, permit, install, and implement design concepts with necessary reviews and approvals. Prepare new or modify existing ADSs.

ACTIONEE: Westinghouse Hanford, DOE-RL, and DOE-HQ

ADS: 9850,9806 , and 9808 
I. Current storage method for waste is not safe or environmentally sound.

B. Potentially explosive ferrocyanide mixture exists in some tanks.

8. Corrective actions have not been implemented to reduce the risk of storage.

NEED:

A demonstration of waste retrieval technology needs to be performed for those tanks where such retrieval is necessary for treatment.

\section{ACTIVITY:}

Develop retrieval technology (both general and Hanford tank specific).

ACTIONEE: Westinghouse Hanford

ADS: $\quad 5178$ 
I. Current storage method for waste is not safe or environmentally sound.

C. Potential reaction of organic and nitrate mixtures in some tanks.

1. No implementation management plan exists.

\section{NEED:}

A plan needs to be prepared with support from management to coordinate the work leading to necessary corrective actions.

\section{ACTIVITY:}

Develop the plan, gain approval and provide management to implement it. Prepare new or modify existing ADSs. Develop the team with Westinghouse, PNL, other national laboratory, subcontractor, and university staffs.

ACTIONEE: Westinghouse Hanford, DOE-RL, and DOE-HQ

ADS: 9852 and 5175 
I. Current storage method for waste is not safe or environmentally sound.

C. Potential reaction of organic and nitrate mixtures in some tanks.

2. All tanks have received many different kinds of waste and the waste needs to be characterized.

\section{NEED :}

The waste in the different tanks needs to be characterized.

\section{ACTIVITY:}

Core sampling rig modification, sampling methods preparation, core sampling, sample preparation and physical properties determination, chemical analysis, robotics development to permit remote sampling, improved remote characterization and preparing supporting safety assessments and environmental assessments.

ACTIONEE: Westinghouse Hanford

ADS: 5175,5176 , and 9852 
I. Current storage method for waste is not safe or environmentally sound. C. Potential reaction of organic and nitrate mixtures in some tanks. 3. Instrumentation must be upgraded and augmented.

\section{NEED:}

Existing instrumentation needs to be upgraded and additional instrumentation needs to be added.

\section{ACTIVITY:}

Upgrade instrumentation tree for temperature and pressure monitoring.

ACTIONEE: Westinghouse Hanford

ADS: 9852 and 9806 
I. Current storage method for waste is not safe or environmentally sound.

C. Potential reaction of organic and nitrate mixtures in some tanks.

4. Requirements have not been established, validated, and implemented for continued safe storage.

NEED:

Requirements need to be established, validated, and implemented for any treatment necessary to allow continued safe storage of the waste until disposal activities are undertaken.

\section{ACTIVITY:}

Identify and validate remediation action requirements and concepts; conduct research development demonstration, testing and elaluation as necessary, evaluate alternatives; establish, permit, install, and implement design concepts with necessary reviews and approvals. Prepare new or modify existing ADSs.

ACTIONEE: Westinghouse Hanford, DOE-RL, and DOE-HQ

ADS: 9852,9806 , and 9808 
I. Current storage method for waste is not safe or environmentally sound.

C. Potential reaction of organic and nitrate mixtures in some tanks.

5. Corrective/remedial action options are not identified/validated.

NEED:

Corrective action needs to be taken to reduce the risk of unsafe storage of waste in the tanks.

\section{ACTIYITY:}

Identity and validate corrective action requirements and concepts; conduct research development demonstration, testing and evaluation as necessary; evaluate alternatives; establish, permit, install, and implement design concepts with necessary reviews and approvals. Prepare new or modify existing ADSs.

ACTIONEE: Westinghouse Hanford, DOE-RL, and DOE-HQ

ADS: 9852 and 9806 
I. Current storage method for waste is not safe or environmentally sound.

C. Potential reaction of organic and nitrate mixtures in some tanks.

6. Technology has not been demonstrated for retrieving waste.

\section{NEED:}

Requirements need to be demonstrated for retrieving the waste for those tanks where such retrieval is necessary for treatment.

\section{ACTIVITY:}

Develop retrieval technology (both general and Hanford tank specific).

ACTIONEE: Westinghouse Hanford and DOE-RL

ADS: 5178 and 5179 
I. Current storage method for waste is not safe or environmentally sound.

C. Potential reaction of organic and nitrate mixtures in some tanks.

7. Corrective actions have not been implemented to reduce the risk of storage.

\section{NEED:}

A demonstration of waste retrieval technology needs to be performed for those tanks where such retrieval is necessary for treatment and alternative corrective actions (e.g., agitation or dilution) need to be evaluated and implemented.

\section{ACTIVITY:}

Develop retrieval technology (both general and Hanford tank specific). Evaluate and implement alternative treatment concepts when validated and appropriate.

ACTIONEE: Westinghouse Hanford

\section{ADS: 5178}


I. Current storage method for waste is not safe or environmentally sound.

D. Continued cooling required for high-heat generation Tank 106-C.

1. Requirements have not been established, validated, and implemented.

\section{NEED:}

Requirements need to be established, validated, and implemented for alternatives to the partial retrieval approach to allow continued safe storage of the waste until disposal activities are undertaken.

\section{ACTIVITY:}

Perform technical evaluation of alternatives to partial retrieval of waste in Tank 106-C; develop, test, and evaluate alternatives as a means of in situ interim stabilization; prepare functional design criteria and environmental assessment; prepare a preliminary safety assessment, conceptual design, definitive design, and safety assessment; construct and implement an alternative if chosen.

ACTIONEE: Westinghouse Hanford, DOE-RL, and DOE-HQ

ADS: 9807,9852 , and 9853 
I. Current storage method for waste is not safe or environmentally sound.

D. Continued cooling required for high-heat generation Tank 106-C.

2. No implementation management plan exists.

NEED :

A plan to address the problem needs to be prepared and management provided to coordinate this work.

\section{ACTIVITY:}

Develop the plan and obtain management support. Prepare new or modify existing ADSs.

ACTIONEE: Westinghouse Hanford

ADS: 9807,9852 , and 5175 
I. Current storage method for waste is not safe or environmentally sound.

D. Continued cooling required for high-heat generation Tank 106-C.

3. Corrective/remedial actions have not been implemented to reduce the risk of storage.

\section{NEED:}

Corrective action needs to be taken to reduce the risk for unsafe storage of the waste currently in Tank 106-C.

\section{ACTIVITY:}

Implement partial retrieval as a means of interim stabilization and implement approach in accordance with all applicable policies and procedures. Develop and implement alternative methods for interim stabilization (e.g., cooling core insertion into tank).

ACTIONEE: Westinghouse Hanford

ADS: 5176 and 9807 
This page intentionally left blank. 
I. Current storage method for waste is not safe or environmentally sound.

D. Continued cooling required for high-heat generation Tank 106-C.

4. If tank leaks, no response capability in the event of a leak.

NEED:

Water has been periodically added to Tank 106-C to keep the sludge wet and to promote heat transfer by evaporation to the vapor space. If Tank 106-C leaks, the need for cooling water would remain. A stabilization approach must be implemented for reducing the heat generation rate, or for cooling the waste without water addition. If the tank leaks, the contents need to be retrieved and transferred to an available double shell tank.

\section{ACTIVITY:}

Continue alternatives study. Characterize the tank contents and develop a model for use in developing and testing a retrieval method if partial or full retrieval is the selected mechanism of interim stabilizing the tank. Upgrade instrumentation for temperature measurement. Upgrade tank ventilation system to enable retrieval. Design, construct, and test retrieval system if iartial or full retrieval is the selected approach. Additional double shell tank space will be required if partial or full retrieval is the selected approach. The environmental consequences will be addressed in NEPA documentation.

ACTIONEE: Westinghouse Hanford

ADS: $9852,9853,9854,9855,9807$ 
1. Current storage method for waste is not safe or environmentally sound.

E. Characterization of tank contents is inadequate.

1. Technology for in situ characterization is not fully developed.

NEED:

Not all analytical requirements can be met and additional analytical methods must be developed. These needs fall into the areas of a designation test, physical property test, and chemical/radionucl:de analyses. The needed methods may not currently exist or may need to be modified for the SST matrices. In some cases testing equipment detection limits need to be improved.

\section{ACTIVITY:}

Criteria must be developed and compared with current laboratory capabilities to identify development needs. (See I.A.8 and III.B.8)

ACTIONEE: Westinghouse Hanford and DOE-RL

ADS: $\quad 5176,9150,9123,9850$, and 9852 
I. Current storage method for waste is not safe or environmentally sound.

E. Characterization of tank contents is inadequate.

2. Core sampling has the potential to initiate chemical reactions.

\section{NEED:}

For tanks identified for the Safety Measures Law, safety and environmental assessments need to be performed to identify the constraints for proceeding with core sampling. For other tanks, adequate review is necessary to assure that potential problems are identified and addressed before core sampling.

\section{ACTIVITY:}

The core sampling rig is being instrumented to ensure drilling temperatures do not exceed predetermined values. Corresponding procedures are being implemented. Historical records of transfers into tanks are being reviewed, and codes updated to assist in identifying contents of concern in each tank, prior to core sampling. For all tanks identified for the Safety Measures Law special precautions are planned, reviewed, and implemented. These include safety and environmental assessments of all planned intrusive activities for the flammable gas mixture and ferrocyanide tanks (Issues I.A and I.B).

ACTIONEE: Westinghouse Hanford

ADS: $9123,9853,9854,9855,5176$ 
I. Current storage method for waste is not safe or environmentally sound.

E. Characterization of tank contents is inadequate.

3. Core sampling has stopped.

NEED:

Successful completion of safety reviews and readiness review for the resumption of core sampling in the push mode is needed. This safety review and readiness review has been impacted by the priority given to sampling Tank $101-S Y$.

The scope and schedule of readiness reviews to address tank contents and vapor space concerns need to be developed. Significant resources are diverted to support the readiness to sample tanks that present a priority safety concern thus impacting completion of SST prerequisite activities.

\section{ACTIVITY:}

Complete Tank 101-SY readiness review.

Complete safety reviews and readiness review for sampling SST waste in push mode. Upgrade safety features of core drill rig for rotary drilling.

Establish and document the scope and schedule of readiness review requirements for tanks that present a safety concern.

ACTIONEE: Westinghouse Hanford

ADS: 5176, 9123, 9850, and 9852 
I. Current storage method for waste is not safe or environmentally sound.

E. Characterization of tank contents is inadequate.

4. Safety issues have not resolved.

\section{NEED:}

Analyses of samples from tanks with priority safety concerns for hydrogen generation, ferrocyanides, and acetates are required to identify remediation steps for tank stabilization and safe operation. The priority established for sampling in support of stabilization of tanks of safety concern will impact SST sampling.

The criteria process must be established and the minimum criteria required to resolve the safety issues must be identified. The criteria process will reduce the number of sampling events as priority safety tanks to a minimum thereby enhancing the SST sampling schedule.

\section{ACTIVITY:}

Complete sampling and analysis of tanks with priority safety concerns to identify remediation steps for stabilization. The characterization needs of other programs must also be integrated in order to use resources effectively and minimized impacts to the other programs.

Establish a formalized criteria process and integrate findings into the sampling and analytical plans and schedules.

ACTIONEE: Westinghouse Hanford

ADS: 9123,9850 , and 9852 
I. Current storage method for waste is not safe or environmentally sound.

E. Characterization of tank contents is inadequate.

5. Integrated tank waste core sampling plan/schedule does not exist.

\section{NEED:}

An integrated site-wide sampling plan and schedule is a prerequisite to identifying the SST sampling schedule. Because of multi-programmatic needs for core samples and analyses, and the priority established for the sampling of tanks that present a safety concern, the SST sampling schedule has been impacted.

\section{ACTIVITY:}

Complete, implement, and maintain formalized and controlled Integrated Tank Waste Core Sampling Plan/Schedule.

ACTIONEE: Westinghouse Hanford

ADS: $5176,9150,9123,9850$, and 9852 
I. Current storage method for waste is not safe or environmentally sound.

E. Characterization of tank contents is inadequate.

6. Lack of approved integrated characterization plan.

\section{NEED:}

The Waste Characterization PIan for the SSTs needs to be revised to encompass the criteria for the tank safety issues (ferrocyanide, organic, and hydrogen SSTS) as well as the criteria for the SST disposal activities. (An integrated DST waste characterization plan (or similar document) also needs to be developed.) Other items to be incorporated into the Waste Characterization Plan include: the characterization strategy/tank sampling order required to support the proposed accelerated SST Closure SEIS and the number. of core per tank based on the decision simulation and impact analysis performed on Phase $1 A / 1 B$ data.

The Waste Characterization Plan for S.JTs is defined as a document primary in the Tri-Party Agreement and is subject to Ecology approval but the document is not yet approved. A plan and schedule needs to be developed for the approval of the Waste Characterization Plan to support the necessary regulator and public reviews of the document.

\section{ACTIYITY:}

Define criteria and tanks to be sampled.

Complete recommendation report from Phase $1 \mathrm{~A} / 1 \mathrm{~B}$ data and balanced data sets.

Revise and issue integrated waste characterization plan.

Develop schedule and plan for State approval of the Waste Characterization Plan.

ACTIONEE: Westinghouse Hanford

ADS: $5176,9150,9123,9850$, and 9852 
I. Current storage method for waste is not safe or environmentally sound.

E. Characterization of tank contents is inadequate.

7. Appropriate sampling methods require development to allow safe tank entry and transfers.

NEED:

To be determined.

\section{ACTIVITY:}

To be determined.

ACTIONEE: To be determined

ADS: To be determined 
I. Current storage method for waste is not safe or environmentally sound.

E. Characterization of tank contents is inadequate.

8. Criteria have not been fully developed.

\section{NEED:}

Criteria and procedures used to define the data requirements for adequate characterization of the wastes are needed. The criteria are the driving force for the waste characterization plans for the methods development.

Identification and integration of multi-programmatic criteria for each sampling event consistent with the integrated core sampling schedule is needed.

\section{ACTIVITY:}

Develop and establish a formal criteria management control system (i.e., plan and implementing procedures).

Implement plan and procedures and define criteria for sampling events (an iterative process) using the products of the criteria process, determine tank contents.

Develop sampling strategy for SSTs to support the accelerated SST Closure SEIS.

ACTIONEE: Westinghouse Hanford

ADS: $5176,9150,9123,9850$, and 9852 
I. Current storage method for waste is not safe or environmentally sound.

E. Characterization of tank contents is inadequate.

9. Inadequate resources (analytical equipment, laboratory capacity, hot cells, core sampling truck, etc.) are available for immediate characterization.

NEED:

Adequate resources are required to complete tank waste core sampling and characterization activities consistent with the integrated tank waste core sampling and analysis plan and schedule. Multi-programmatic funding adjustments are required to take maximum advantage of the sampling events scheduled in the integrated plan.

Baseline definition and funding for the laboratories and support groups consistent with the integrated core sampling plan and schedule to ensure adequate manpower, equipment upgrades and modifications, analytical methods and database development, and adequate sampling equipment and spare parts are required.

Capital equipment funding to support acquisition of major equipment items (e.g., Laboratory Information Management System data management system, radioanalytical instrumentation, core sampling trucks and ancillary equipment, etc.) to significantly improve laboratory and field sampling throughput/capability must be provided. Funding is also needed to ensure completion of the hot cell upgrade project at the earliest possible date and for other major projects that would significantly increase laboratory core sample processing capability.

\section{ACTIVITY:}

Prepare/approve integrated characterization plan.

Adjust/establish funding for tank waste core sampling and analys is and other characterization activities consistent with the integrated schedule.

Establish long-term funding to support accelerated sampling and analysis schedules.

Establish long-term funding and supporting expense funding to ensure completion of major upgrades such as the new hot cells.

ACTIONEE: Westinghouse Hanford, DOE-RL, and DOE-HQ

ADS: 5250,9446 , and 9900 
I. Current storage method for waste is not safe or environmentally sound.

F. Safety documentation for the SSTs is less than adequate.

1. Changes in the DOE Orders have occurred and Operational Safety Requirements have not been revised.

\section{NEED:}

Upgrade of the SST operating safety requirements is needed to assist in the definition of the safety envelope and aid in compliance with DOE orders.

ACTIVITY:

Prepare an upgrade to the SST operating safety requirements.

ACTIONEE: Westinghouse Hanford

ADS: 9853 
I. Current storage method for waste is not safe or environmentally sound.

F. Safety documentation for the SSTs is less than adequate.

2. Changes in the DOE Orders have occurred and the Safety Analys is Report has not been revised.

\section{NEED:}

Upgrade of the SST safety analysis report is needed to assist definition of the safety envelope for the SST facility and aid in compliance with DOE orders.

ACTIVITY:

Prepare an upgrade to the SST safety analysis report; prepare an addendum to the SST safety analysis report to cover overland transfers.

ACTIONEE: Westinghouse Hanford

ADS: 9853 
I. Current storage method for waste is not safe or environmentally sound.

G. Maintenance facilities, equipment, and instrumentation has been deficient (upgrades are required).

1. Control room and work spaces are inadequate.

NEED:

Need to provide an improved control room and maintenance, engineering, and administrative work space.

ACTIVITY:

Design and construct a modern, clean, well lighted control room.

Design and construct maintenance, engineering, and administrative work space for 200 East and 200 West tank facilities.

ACTIONEE: Westinghouse Hanford

ADS: 9803 
I. Current storage method for waste is not safe or environmentally. sound.

G. Maintenance facilities, equipment, and instrumentation has been deficient (upgrades are required).

2. Operational support equipment is less than adequate.

NEED:

Need to provide upgrade equipment.

\section{ACTIVITY:}

Provide new upgraded air compressors, emergency standby diesel power; portable exhausters; transportable impact wrench stations; pump and pump jumper storage racks; improved (repaired and/or replaced) alarms; liquid level, leak detection, and temperature sensors and/or recorder replacements; new ventilation monitoring systems; and ammonia monitors.

ACTIONEE: Westinghouse Hanford

ADS: 9803 
I. Current storage method for waste is not safe or environmentally sound.

G. Maintenance facilities, equipment, and instrumentation has been deficient (upgrades are required).

3. Buildings and equipment require cleanup and/or decontamination.

NEED:

Need to remove and decontaminate equipment and facilities.

ACTIVITY:

Remove unused electrical, instrument, piping and mechanical equipnent. Remove unused buildings. Decontaminate facilities that are planned for continued use and require decontamination.

ACTIONEE: Westinghouse Hanford

ADS: 9803 


\section{$D O E / R L-90-48$}

Predecisional Draft

I. Current storage method for waste is not safe or environmentally sound.

G. Maintenance facilities, equipment, and instrumentation has been deficient (upgrades are required).

4. Facilities contain asbestos.

\section{NEED:}

Need to remove asbestos and implement a painting program.

\section{ACTIVITY:}

Continue to remove asbestos and replace with approved insulation. Implement a painting program for change rooms, rest rooms, and other tank farm facilities.

ACTIONEE: Westinghouse Hanford

ADS: 9803 
I. Current storage method for waste is not safe or environmentally sound.

G. Maintenance facilities, equipment, and instrumentation has been deficient (upgrades are required).

5. Leak detection is less than adequate.

NEED:

Need to provide state-of-the-art leak detection systems for the detection capability of 2 kilograms (or about 2 gallons) as required by RCRA.

\section{ACTIVITY:}

Complete installation of liquid observation wells in tanks where needed. Perform and engineering study to determine whether alternative state-of-theart leak detection and liquid level monitoring methods exist. This will include a vendor search to determine whether commercial products are available. Possible alternatives include other instrumentation that can be installed in liquid observation wells.

Develop state-of-the-art leak detection systems for SSTs. Use available commercial equipment that; modify for use in SSTs. Perform in-service tests on capabilities of equipment in one or more tanks.

Design, procure, fabricate, and install equipment upgrades to improve lead detection and liquid level monitoring in all 149 SSTs.

Prepare detailed procedures and training packages. Train operators and maintenance and engineering personnel on new leak detection equipment.

ACTIONEE: Westinghouse Hanford

ADS: 9302 
I. Current storage method for waste is not safe or environmentally sound.

G. Maintenance facilities, equipment, and instrumentation has been deficient (upgrades are required).

6. Instrumentation is less than adequate.

NEED:

Need to upgrade instrumentation capability to state-of-the-art. It must have three-dimensional capability, be highly responsive, and must be able to analyze data to meet today's standards.

\section{ACTIVITY:}

Develop a fully integrated upgrade plan for the tank farms that is based on the instrument upgrade activities currently being planned for issues I.A, I.B, I.C, and I.D.

Design, construct, and start projects to provide for a fully modernized tank farm instrumentation systems.

Perform the necessary front-end engineering work to support the electrical upgrade capital projects that are required to provide adequate power distribution for the new instrumentation system. Design, construct, and start the projects to provide a modernized distribution system for the instrument upgrade projects.

ACTIONEE: Westinghouse Hanford

ADS: 9806 
1. Current storage method for waste is not safe or environmentally sound.

G. Maintenance facilities, equipment, and instrumentation has been deficient (upgrades are required).

7. S-302-A catch tank leaks.

NEED:

Repair S-302-A catch tank to provide secondary containment for the S-151 diversion box, the common transfer point in the 200 West Area. Alternatively, a replacement or alternative means of liquid transfer needs to be established.

\section{ACTIVITY:}

Grout the bottom one-third of the S-302-A catch tank to plug the leak. (This type of grout "repair" of an underground, inaccessible tank had never been attempted before.) The U.S. Army Corp of Engineers was contacted and aided Westinghouse Hanford in the formulation of low viscosity, zero shrink grout that would meet all the technical criteria and successfully seal the leaking area of this tank. This grouting has been completed. If the tank leak test is successful, the tank will be placed back in service until the replacement tank can be installed.

Design and replace the S-302-A catch tank with a new, fully compliant (DOE Order, EPA, State of Washington) tank. This project is $W-205, S-302-A$ catch tank. It is planned as a FY 1991 general plant project.

ACTIONEE: Westinghouse Hanford

ADS: 9805 
I. Current storage method for waste is not safe or environmentally sound.

G. Maintenance facilities, equipment, and instrumentation has been deficient (upgrades are required).

8. Concrete containment for underground transfer lines not acceptable per regulations.

\section{NEED:}

Need to evaluate system and provide adequate containment in compliance with current regulations.

\section{ACTIVITY:}

Perform integrity assessments to ensure that the tank system is fit for use. The transfer facilities that are designed with adequate secondary containment will be assessed for integrity as part of the integrity assessment. The assessment will include comparison of as-built standard to today's standards, evaluation of structural integrity in the waste environment, and leak testing and inspecting where practical.

Replace all transfer lines that are active or planned to be active, where concrete is used as a secondary containment method, for pipe-in-pipe systems. Transfer facilities that-do not comply with secondary containment requirements will have operational testing performed before and during transfers. Mass balances and leak detection for transfers ensure that the ongoing transfers are accomplished safely. Leak testing of transfer piping that has not been used within the previous year is another way to ensure the integrity of transfer routes. Replacements to existing transfer facilities that do not contain adequate secondary containment will be evaluated as part of the acceptance for upgrades to the respective facilities.

ACTIONEE: Westinghouse Hanford

ADS: 9806 and 9119 
I. Current storage method for waste is not safe or environmentally sound.

H. SSTs have exceeded their original operating life.

1. Steel and concrete aging evaluations are incomplete.

NEED :

Each tank must be considered at least somewhat "suspect" in terms of tank and waste form stability until determined differently. A process is needed to validate and document the acceptability of continued SST storage and the SST remediation program.

\section{ACTIVITY:}

Define process and draft criteria; review and obtain concurrence from external groups including interested States and Indian Nations; verify tanks structurally and in terms of tank controls.

ACTIONEE: Westinghouse Hanford

ADS: 9853 
1. Current storage method for waste is not safe or environmentally sound.

H. SSTs have exceeded their original operating life.

2. Estimating remaining structural life of SSTs does not exist.

\section{NEED:}

Need to evaluate material property aging.

\section{ACTIVITY:}

Quantify material property aging. Specifically, review the corrosion or degradation of the reinforcing steel and the concrete matrix. No additional evaluations of the SST liners will be conducted because it is assumed that they have already leaked. Samples of the concrete have been taken, but additional sampling and testing of the tank concrete may be warranted. A specific measurement regime will be developed and implemented to detect potential structural problems. Parametric studies determine which critical properties should be considered in a surveillance and inspection plan.

ACTIONEE: Westinghouse Hanford

ADS: 9853 
I. Current storage method for waste is not safe or environmentaliy sound.

H. SSTs have exceeded their original operating life.

3. Approximately one-half of the SSTs leak, the others could leak at any time.

\section{NEED:}

Need to estimate tank lifetimes.

\section{ACTIVITY:}

The task of analyzing tank life expectancy is intended to provide reasonable lifetime estimates for either specific tanks or unique tank groups.

structural analyses will be performed, and the results will be applied to support tank life expectancy estimates. A key component of this task is to quantify the effects of the environment on material properties. Structural analyses and evaluations will be performed to support the tank life expectancy evaluation. Either unique or bounding in situ and environmentally affected material properties will be applied.

ACTIONEE: Westinghouse Hanford

ADS: 9853 
I. Current storage method for waste is not safe or environmentally sound.

H. SSTs have exceeded their original operating life.

4. Structural acceptable life expectancy of SSTs must be determined.

NEED:

Concerns have been raised regarding seismic capacity and concrete material property aging of the tank structures, especially for tanks that have been structurally "abused."

\section{ACTIVITY:}

Conduct a tank structural analysis for all tank designing, evaluating thermal cycling, life loads, seismic loads, etc. the tanks have been subjected or may be subjected to. Assess and analyze material aging. Implement tank structural property monitoring program. Asses tank acceptable life. Prepare new or modify existing ADSs.

ACTIONEE: Westinghouse Hanford

ADS: 9853 
I. Current storage method for waste is not safe or environmentally sound.

H. SSTs have exceeded their original operating life.

5. Extensive contaminated soil from prior spills and leaks.

\section{NEED :}

The leak plumes in the SST farms need to be re-investigated to respond to General Accounting Office Audit Report No. RCED-89-157. This investigation/ characterization needs to be integrated with work necessary to satisfy SEIS and RCRA closure data requirements.

SST operable units needs to be characterized to satisfy the technical and schedule requirements for SST SEIS Closure.

\section{ACTIVITY:}

Complete the SST System Closure/Corrective Action Work Plan and establish level of characterization necessary to satisfy technical and schedule requirements for SST SEIS Closure.

Prepare summary plan and work plan for SST operable units field characterization, obtain approvals and-implement.

ACTIONEE: Westinghouse Hanford

ADS: 5175 
I. Current storage method for waste is not safe or environmentally sound.

I. Emergency pumping capability is deficient.

1. Double-contained receiver tanks do not exist for two tank farms.

NEED:

Need to provide double containment for $244-C R$ and $244-V$.

\section{ACTIVITY:}

Refurbish the 244-CR Facility so that saltwell liquid can be received, and complete the operability tests and readiness review. Complete the installation of equipment at the 244-U double contained receiver tank, verify the operability of the facility; and perform a readiness review.

ACTIONEE: Westinghouse Hanford

ADS: 9305 
I. Current storage method for waste is not safe or environmentally sound.

I. Emergency pumping capability is deficient.

2. Overground transfer equipment is lacking.

NEED :

Need to design, procure, fabricate, and prepare equipment for operation.

\section{ACTIVITY:}

Conduct an engineering study; design criteria; and design, procure, and fabricate overground transfer equipment to perform an overground transfer as needed.

Prepare detailed procedures showing exactly how each tank will be pumped, where it will be pumped to, and exactly what equipment will be used and where that equipment is stored. Put a hold on all the equipment so it cannot be used for some other activity. Prepare all the readiness reviews and other documentation so that no approvals will be needed to execute the overground pumping procedures.

Prepare all necessary training packages, train a group of people (including operators, maintenance and engineering personnel), and conduct periodic practice sessions to ensure that all people know how to install and use the necessary equipment and procedures for pumping an SST.

Provide an addendum to the SST safety analysis report to support the overground transfer.

ACTIONEE: Westinghouse Hanford

ADS: 9305 and 9853 
I. Current storage method for waste is not safe or environmentaliy sound.

I. Emergency pumping capability is deficient.

3. Emergency pumping units do not exist.

NEED:

Need to provide emergency pumping units.

\section{ACTIVITY:}

Procure, fabricate, and assemble units to be used in emergency pumping for a faster response. Have the capability to respond to pumping an unstabilized leaking tank within 7 days.

ACTIONEE: Westinghouse Hanford

ADS: 9305 
I. Current storage method for waste is not safe or environmentally sound.

J. Potential exists for toxic vapor release.

1. Nonradioactive chemical characterization data for waste tank vapor spaces, specifically on the presence of toxic vapors, does not exist.

\section{NEED :}

Need to characterize vapors, because the tank vapors may pose a personnel hazard to those working in the area. Also, RCRA release limits may be exceeded.

\section{ACTIVITY:}

Complete a vapor sampling plan for the tank farms. The main purpose of the plan is to identify potential health and safety hazards to prevent occupational disease or injury. Implement sampling to define the scope of the problem.

Characterize the vapors in the tank dome space that are generated by the tank waste. Determine if any of the vapors pose an occupational health and safety hazard.

ACTIONEE: Westinghouse Hanford

ADS: 9302 
I. Current storage method for waste is not safe or environmentally sound.

J. Potential exists for toxic vapor release.

2. Interim remediation methods do not exist.

\section{NEED:}

Need to develop methods for mitigating consequences of toxic releases.

\section{ACTIVITY:}

Develop methods for mitigating the consequences of toxic release, such as increasing stack heights on ventilation systems, installation of monitors, different types of filters, increasing vent flow rates and installation of more vents. Also, the prevention of vapor generation may be an effective option.

The focus at the beginning will be the Safety Measures Law Identified tanks.

Finalize requirements, perform front end engineering studies, including NEPA documentation. Design, construct and/or procure and start up toxic detection/prevention system.

ACTIONEE: Westinghouse Hanford

ADS: 9302 
I. Current storage method for waste is not safe or environmentally sound.

J. Potential exists for toxic vapor release.

3. All release paths are not filtered and monitored.

NEED:

Need to ensure that all tanks determined (by vapor sampling or operating and historical records) to have the potential for toxic vapor release have all their release paths monitored and rendered nonhazardous to prevent occupational disease or injury. Ventilation and monitoring upgrade activities need to include this issue as part of their functional design criteria.

\section{ACTIVITY:}

Vapor sampling and review of operating and historical records will lead to identification of candidate tanks. Functional design criteria for monitoring and ventilation upgrades shall include addressing the corrective action.

closure of this issue.

ACTIONEE: Westinghouse Hanford with concurrence by Hanford Environmental
Health Foundation

ADS: $9108,9853,9123,9850,9852,9805$, and 9806 
This page intentionally left blank.

- 
DOE/RL- $90-48$

Predecisional Draft

$4 / 5 / 91$

I. Current storage method for waste is not safe or environmentally sound.

K. Conduct of operations and administrative systems are less than adequate.

1. Procedure system is less than adequate.

NEED :

Need to upgrade procedure system.

\section{ACTIVITY:}

Westinghouse Hanford guidelines will be written using INPO good practices, DOE Orders on conduct of operations and/or off-normal event reporting, and

industry standards. Plant guides for procedure writers will be upgraded to reflect a new upgrade standard. All procedures will be reviewed and rewritten in accordance with new standards.

Several upgrades will be made to the procedure control system to ensure that operators are always using the latest revision.

ACTIONEE: Westinghouse Hanford

ADS: 9100 
I. Current storage method for waste is not safe or environmentally sound.

K. Conduct of operations and administrative systems are less than adequate.

2. Training programs require further development.

NEED:

Need to upgrade training programs.

\section{ACTIVITY:}

Upgrade the on-the-job training program; develop a training evaluation program; develop new training material; begin formal classroom training to enhance the present self-study program; and evaluate the supervisor and manager plant-specific training to ensure it is meeting the identified needs.

ACTIONEE: Westinghouse Hanford

ADS: 9100 
I. Current storage method for waste is not safe or environmentally sound.

K. Conduct of operations and administrative systems are less than adequate.

3. Tank farm drawings have not been maintained and are inaccurate.

\section{NEED:}

Need to provide correct/current as-built engineering drawings in compliance with DOE Orders.

\section{ACTIVITY:}

Identify and prioritize those drawings that need to be verified and the drawings that can be consolidated or superseded to eliminate existing configuration and control problems. For those drawings that need upgrading, verify and convert them to computer-aided design and drafting data sets, and incorporate all outstanding Engineering Change Notices.

Existing flow diagrams will be upgraded to enhance their usefulness in troubleshooting operational problems. New schematic drawings will be created for training piant operators and support staff.

Develop a Master Component Index consisting of the attributes used to define an individual part or component. Significant attributes such as operating limits and/or specifications, acceptable substitute replacement parts, special tools or maintenance requirements, dimensions, and more generic data, such as component name, number, and location, will be included in the index.

ACTIONEE: Westinghouse Hanford

ADS: 9804 
I. Current storage method for waste is not safe or environmentally sound.

L. Inadequate DST space to support waste management needs.

1. Need to keep waste types segregated.

NEED:

Different waste types must be kept segregated to prevent adverse interactions, and to permit subsequent cost effective pretreatment of the waste for final disposal.

\section{ACTIVITY:}

Finalize requirements, perform front-end engineering studies, including NEPA documentation. Design, construct, and start new tank farm.

ACTIONEE: Westinghouse Hanford

ADS: 9806 and 9851 
I. Current storage method for waste is not safe or environmentally sound.

L. Inadequate DST space to support waste management needs.

2. Insufficient space to support SST pumping.

\section{NEED :}

Need to determine space needs and provide new capacity:

\section{ACTIVITY:}

Identify tank capacity needs for the removal of SST waste to remediate the potential leakage to the environment. Tank capacity versus through-put to disposal requires tradeoff studies before selecting the number of tanks to be built.

Finalize requirements, perform front-end engineering studies, including NEPA documentation. Design, construct, and start new tank farm.

ACTIONEE: Westinghouse Hanford, DOE-RL, and DOE-HQ

ADS: 9806 and 9851 
I. Current storage method for waste is not safe or environmentally sound. L. Inadequate DST space to support waste management needs.

3. Insufficient space to support remediation actions in support of safety issues and stabilization of Tank 106-C.

NEED:

Current corrective actions for Tank 101-SY episodic gas release, and cooling requirements of Tank 106-C, favor transfer of some of the waste from these tanks into other tank space. Such space is not available.

\section{ACTIVITY:}

Finalize requirements, perform front-end engineering studies, including NEPA documentation. Design, construct, and start new tank farm.

ACTIONEE: Westinghouse Hanford

ADS: To be determined 
I. Current storage method for waste is not safe or environmentally sound. M. Infittrations into SSTs are possible.

1. Not all SSTs have been stabilized and isolated.

NEED:

Need to complete stabilization and isolation program.

\section{ACTIVITY:}

Expedite the interim stabilization (saltwell pumping) and interim isolation program for the remaining SSTs. For those tanks awaiting interim isolation, investigate and implement methods to reduce intrusions until saltwell pumping is completed.

ACTIONEE: Westinghouse Hanford

ADS: 9305 
I. Current storage method for waste is not safe or environmentally sound. M. Infiltrations into SSTs are possible.

2. Some liquid infiltrations are occurring in interim isolated SSTS.

NEED:

Need to study the situation and provide the needed upgrades.

ACTIVITY:

Perform engineering studies to determine why some liquid intrusions are still occurring and evaluate additional methods to eliminate intrusions. Develop requirements, and perform front-end engineering studies, including NEPA documentation. Design, construct, and install upgrades to prevent intrusions for all interim isolated SSTs.

ACTIONEE: Westinghouse Hanford

ADS: 9305 
I. Current storage method for waste is not safe or environmentally sound. M. Infiltrations into SSTs are possible.

3. Infiltration may eventualiy leak back out with potential for transport of wastes.

\section{NEED:}

In addition to upgrading the prevention of infiltration into SSTs

(Issue I.M.2), improved detection for identifying slow leaks of this liquid back into the soil with the associated transport of waste is needed.

\section{ACTIVITY:}

The actions identified in Issue I.G.5 will address this issue.

ACTIONEE: Westinghouse Hanford

ADS: 9302 
This page intentionally left blank.

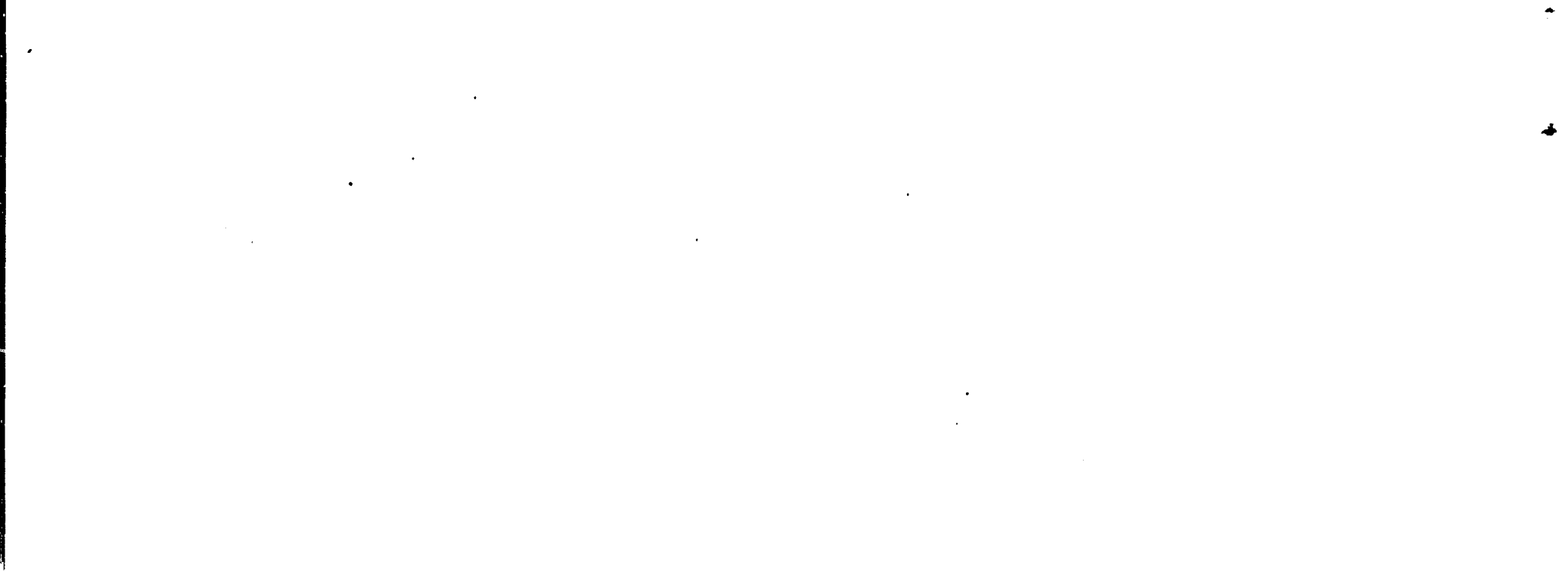


I. Current storage method for waste is not safe or environmentally sound.

N. Process and criteria are needed to validate all SSTs ready for remediation.

1. No formal criteria published to review tanks against to ensure it's safe to turn them over to restoration.

\section{NEED:}

It is important that the criteria that all SSTs are to be reviewed against before releasing to environmental restoration be formalized.

\section{ACTIVITY:}

Document the criteria used to date to review the SSTs for safety issues.

Identify other criteria that should also be applied to SSTs.

Ensure adequate internal and external peer review of the criteria.

Publish document.

ACTIONEE: Westinghouse Hanford

ADS: 9853 
I. Current storage method for waste is not safe or environmentally sound.

N. Process and criteria are needed to validate all SSTs ready. for remediation.

2. Insufficient documented historical data and chemical review.

NEED:

In order to assure that there are no unacceptable safety or environmental issues associated with individual tanks, it is important to understand the operating history of the tanks (e.g., transfers, wastes added or removed, waste temperatures, etc.). Much of the early history of the tanks is either not well documented or the documentation is not readily retrievable.

\section{ACTIVITY:}

Improve the retrievability of existing historical documentation (review information in long term storage for potential safety issues; at same time, enter key information into database to assist in future retrievability of the data).

Perform additional interviews with retirees from tank farms to "fill in the gaps" of the written records.

Centralize all the chemical information (transfer amounts, grap sample analyses, etc.) and place in form to allow better review by technical experts.

Upgrade predictive model (based on transfer histories, mass balance, etc.) of tank contents.

Ensure new data, and to extent possible historical data, is stored and archived in manner allowing maximized electronic transfer of information to facilitate reviews.

ACTIONEE: Westinghouse Hanford

ADS: 9853 
I. Current storage method for waste is not safe or environmentally sound.

N. Process and criteria are needed to validate all SSTs ready for remediation.

3. Data must be assembled and packaged to allow external review and audit.

NEED:

Given the importance of this review, it is important that the data be properly assembled and packaged to allow other organizations to review and comment on the data and the assumptions and conclusions made. These "packages" must be available for external groups who request them to be able to obtain the information.

\section{ACTIVITY:}

Agree on format the information is to be best presented in.

Agree on the backup file media.

Collect the historical data necessary.

Document assumptions and conclusions.

Publish the packages (tank by tank) for peer review.

Issue the final review packages.

ACTIONEE: Westinghouse Hanford

ADS: 9853 
DOE/RL-90-48

Predecisional Draft

$4 / 5 / 91$

This page intentionally left blank. 
DOE/RL- $90-48$

Predecisional Draft.

$4 / 5 / 91$

\subsection{ISSUE II - ABILITY TO MEET COMMITTED CLOSURE} MILESTONES IS UNCERTAIN 
This page intentionally left blank. 


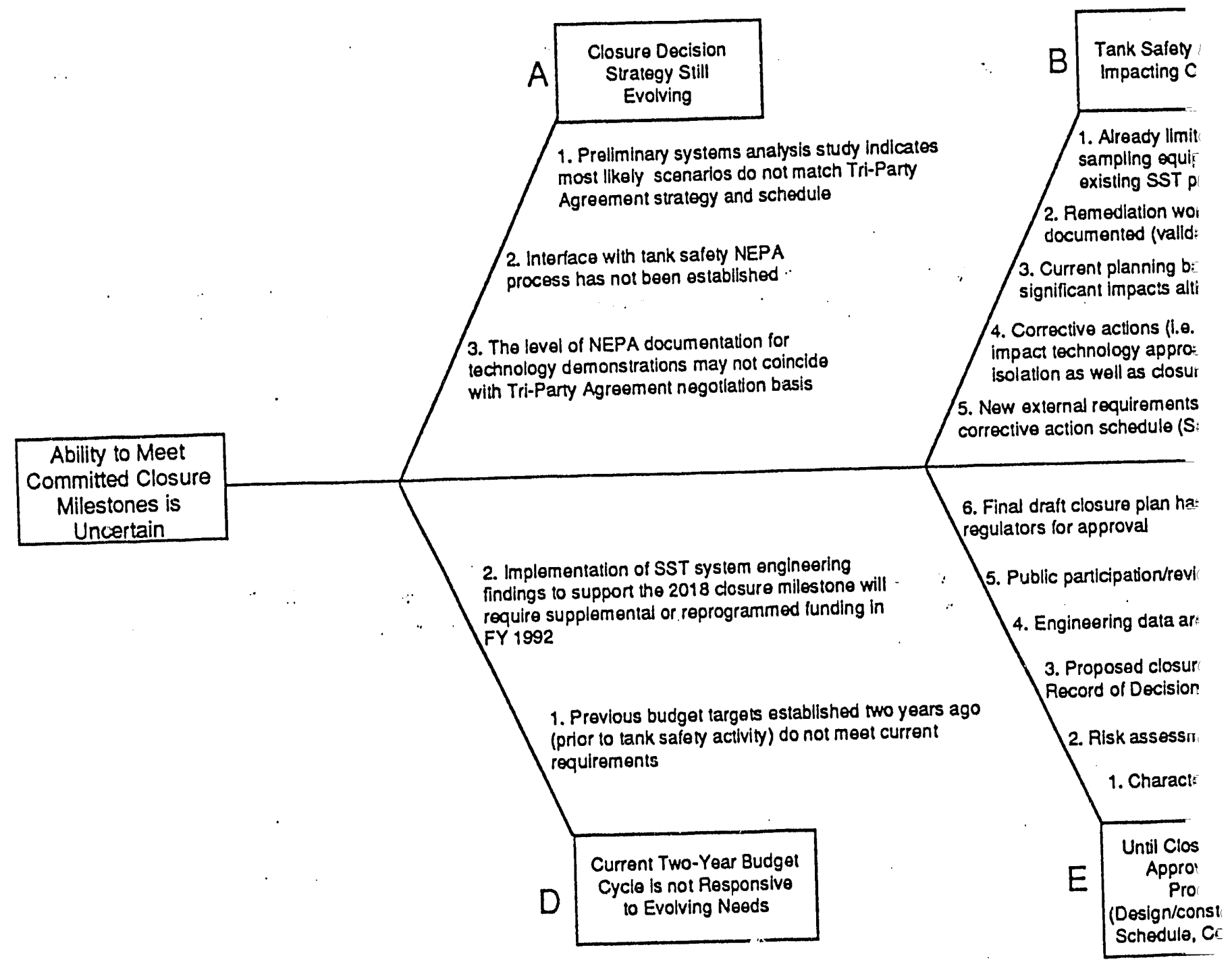

2. Implementation of SST system engineering findings to support the 2018 closure milestone will require supplemental or reprogrammed funding in FY 1992

1. Previous budget targets established two years ago (prior to tank safely activity) do not meet current requirements to Evolving Needs Schodule, Cc 
d resources (personnel, analytlcal capacity, hent, funding etc.) have been diverted from bram to tank safery program

is suspended until formal review is (lon)

$\theta$ does not anticipate future new discoveries with ough future reviews are a certainty

otrieval/treatment of ferrocyanide) may

$h$ and schedule for stabilization and analysis to optimize program

re driving elements of tank safery bry Measures Law)

5. Schedule for availability of repositories impacts disposal of DST waste and may impaci disposal of SST wasio disposal

hot been prepared and submitted to

7. Design, permitring, construction of the retrieval option does not support the 2018 committed milestone

6. Corrective action under $3004(\mathrm{U})$ may be unable to support an accelerated

has not been Initiated SST Closure SEIS

pomplete

-

ction selection is dependent upon NEPA

$t$ is not complete

ation of waste and operable units is not complete 5. SST Closure SEIS review and approval schedule is not intemally controlled 4. Interface with tank safery NEPA process has not been established

3. Waste destruction, stabilization lechnology, and burial performance required by HDW-EIS Record of Decision has not been completed

2. Waste retrieval \& treatment technology evaluation required by HDW-EIS Fecord of Decision has not been completed

Plan is Prepared and the Technical and inmatic Baseline uction/operation Scope, st) Cannot be Finalized
1. Characterization identifled by HOW-EIS Record of Decision has no been completed

$F \quad \begin{gathered}\text { Until SST Closure SEIS is } \\ \text { Prepared, Approved and ROD } \\ \text { Issued, SST Program Cannot } \\ \text { Restrict Options and Optimize } \\ \text { Schedule }\end{gathered}$


II. Ability to meet committed closure milestones is uncertain.

A. Closure decision strategy still evolving.

1. Preliminary systems analysis indicates most likely scenarios do not match Tri-Party Agreement strategy and schedule.

NEED :

Design, permitting, and construction of facilities required for closure and postclosure activities (including monitoring and potential treatment of retrieved waste) need to be included in planning basis.

\section{ACTIVITY:}

Develop technical basis for closure strategy and schedule using the systems analysis study. Revise strategy in conjunction with regulators. Changes to the schedule will be made as appropriate using the established Tri-Party Agreement process.

ACTIONEE: Westinghouse Hanford, DOE-RL, and DOE-HQ

ADS: $\quad 5175$ 
II. Ability to meet committed closure milestones is uncertain.

A. Closure decision strategy still evolving.

2. Ir.terface with tank safety NEPA process has not been established.

\section{NEED:}

Need to determine the scope of the safety NEPA document and establish interface with Closure SEIS.

ACTIVITY:

Begin pre-scoping activities.

ACTIONEE: Westinghouse Hanford, DOE-RL, and DOE-HQ

ADS: 9855 
II. Ability to meet committed closure milestones is uncertain.

A. Closure decision strategy still evolving.

3. The level of NEPA documentation for technology demonstration may not coincide with Tri-Party Agreement negotiation basis.

\section{NEED:}

A determination is required whether to prepare Environmental Assessments or EIS on technology demonstration commitments. The negotiation assumed NEPA would be applicable but an EIS would not be required.

\section{ACTIVITY:}

Determine appropriate level of NEPA documentation required for technology demonstrations.

ACTIONEE: DOE-HQ

ADS: To be determined 
II. Ability to meet committed closure milestones is uncertain.

B. Tank safety activities are impacting commitments.

1. Already $1 i_{\ldots i}$ ited resources (personnel, analytical capacity, sampling equipment, funding, etc.) have been diverted from existing SST program to tank safety program.

NEED:

Supplemental and/or reprogrammed funds are required in FY 1991 and FY 1992 to address tank safety requirements.

Adequate resources are required to complete tank waste core sampling and characterization activities consistent with the integrated tank waste core sampling and analysis plan and schedule. Multi-programmatic funding adjustments are required to take maximum advantage of the sampling events scheduled in the integrated plan.

Baseline definition and funding for the laboratories and support groups consistent with the integrated core sampling plan and schedule to ensure adequate manpower, equipment upgrades and modifications, analytical methods and database development, and adequate sampling equipment and spare parts are required.

Capital equipment funding planned to support acquisition of major equipment items (e.g., Laboratory Information Management System, radioanalytical instrumentation, core sampling trucks and ancillary equipment, etc.) to significantly improve laboratory and field sampling throughput/ capability must be provided. Funding is also needed to ensure completion of the hot cell upgrade project at the earliest possible completion date and for other major projects that would significantly increase laboratory core sample processing capability.

\section{ACTIVITY:}

Prepare/approve integrated characterization plan.

Adjust/establish funding for tank waste core sampling and analys is and other characterization activities consistent with the integrated schedule.

Establish long-term funding to support accelerated sampling and analysis schedules.

Establish long-term funding and supporting expense funding to ensure completion of major upgrades such as the new hot cells. 
DOE/RL - $90-48$

Predecisional Draft

$4 / 5 / 91$

ACTIONEE: Westinghouse.Hanford, DOE-RL, and DOE-HQ ADS: 5250,9446 , and 9900 
II. Ability to meet committed closure milestones is uncertain.

B. Tank safety activities are impacting commitments.

2. Remediation work is suspended until formal review is documented (validation).

NEED:

Complete validation, proceed with tank waste characterization, and laboratory studies.

ACTIVITY:

Proceed with validation, tank waste characterization, and laboratory studies in acrordance with integrated characterization and laboratory analyses plans.

ACTIONEE: Westinghouse Hanford, DOE-RL, and DOE-HQ

ADS: $9806,9850,9853,9852$, and 9123 
II. Ability to meet committed closure milestones is uncertain.

B. Tank safety activities are impacting commitments.

3. Current planning does not anticipate future new discoveries with significant impacts al though future reviews are a certainty.

NEED:

Proactive identification of potential safety issues.

ACTIVITY:

Comprehensive review of all tanks and evaluation/analysis of potential reaction mechanisms for timely incorporation into planning.

ACTIONEE: Westinghouse Hanford

ADS: 9853 
II. Ability to meet committed closure milestones is uncertain.

B. Tank safety activities are impacting commitments.

4. Corrective action (i.e., retrievable/treatment of ferrocyanide) may impact technology approach and schedule for stabilization and isolation, as well as closure.

NEED:

Prompt closure of safety issues many not be possible when line item projects, major projects, or major system acquisitions are required to provide new systems and/or facilities for issue closure. Exemptions from the budget cycle process, or funding that can be used for either expense or capital work must be made available as soon as the work can be accurately defined and started.

The existing Tri-Party Agreement milestone for completion of interim stabilization and isolation needs to take into account that continued addition of water and/or other chemicals, continued ventilation, continued monitoring, and/or retrieval of the waste for subsequent treatment may be required to ensure safety interim storage. Some tanks that have been stabilized and isolated may need to be reopened for actions listed above.

\section{ACTIVITY:}

Complete development of waste tank safety issue closure planning and identify required projects; review potential groupings of projects into major system acquisitions to lock in adequate out-year funding; allow major system acquisition validation based on initial statements of need and engineering individual sub-projects; explore means to establish congressional support for closure of waste tank safety issues with funding free from expense versus capital constraints; and follow the standard DOE acquisition process but eliminate the 2-year delay from completion of conceptual design to start of capital-funded work.

Complete tank sampling and analysis tasks; complete safety analyses; complete safety issue resolution plans; and renegotiate the specific terms for the subject Tri-Party Agreement milestone for those tanks which cannot be interim stabilized to achieve a safe interim storage mode. For those tanks that are now isolated, reopen and take steps as required to place the waste in a safe interim storage mode.

ACTIONEE: Westinghouse Hanford, DOE-RL, and DOE-HQ

ADS: $9176,9123,9850,9851,9852,9853,9854,9855,9806$, and 9808 
II. Ability to meet committed closure milestones is uncertain.

B. Tank safety activities are impacting commitments.

5. New external requirements are driving elements of tank safety corrective action schedule (Safety Measures Law).

NEED:

Satisfy new external requirements and mitigate impacts to existing committed milestones.

\section{ACTIVITY:}

Safety Issue Corrective Action Plans must be prepared in response to the new requirements. These plans will include the technical basis, strategy, planning, and require concurrence on proposed actions and schedules. Assessment of impacts will require participation of regulators and the public. Resulting changes to the Tri-Party Agreement schedule will be made as appropriate using the established Tri-Party Agreement process.

ACTIONEE: Westinghouse Hanford, DOE-RL, and DOE-HQ

ADS: $9123,9850,9851,9852,9853,9854$, and 9855 
II. Ability to meet committed closure milestones is uncertain.

C. SST program is not integrated.

1. No single point of authority, accountability, responsibility (SST cross many organizations and programs. at DOE-HQ, DOE-RL, and Westinghouse Hanford).

NEED:

Identify single point of authority, accountability, and responsibility and a direct chain of command.

\section{ACTIVITY:}

Review organizational structures and consolidate as required to meet need.

ACTIONEE: Westinghouse Hanford, DOE-RL, and DOE-HQ

ADS: To be determined 
DOE/RL $-90-48$

Predecisional Draft

$4 / 5 / 91$

II. Ability to meet committed closure milestones is uncertain.

C. SST program is not integrated.

2. There is not an approved technology development baseline (integrated demonstration).

NEED:

Timely and sufficiently detailed Integrated Demonstration Plan.

\section{ACTIVITY:}

Westinghouse Hanford must prepare the Integrated Demonstration Plan and obtain timely approval by DOE-RL and DOE-HQ to allow integration with Environmental Restoration and Waste Management plans for 1992 and 1993.

ACTIONEE: Westinghouse Hanford, DOE-RL, and DOE-HQ

ADS: 9711 
II. Ability to meet committed closure milestones is uncertain.

C. SST program is not integrated.

3. Integrated baseline $\mathrm{plan} / \mathrm{schedule}$ does not exist in sufficient detail.

There are three programs involved in activities directed towards interim storage/surveillance of SSTs, closure of operable units, and disposal of waste that may be retrieved from the SST. These programs are Waste Management, Environmental Restoration, and Technology Development.

NEED:

Programmatic and functional responsibilities need to be established and a lead responsible program identified to integrate and coordinate (scope, budget, and schedule) the overall SST program.

A formal and controlled integrated SST program baseline plan/schedule, including the identification of scheduling constraints, needs to be developed. This baseline $\mathrm{plan} / \mathrm{schedule}$ needs to address resources necessary to meet TriParty Agreement milestones, to resolve issues identified in the Hanford Site SST Roadmap and to apply the SST System Engineering approach for closure of SST operable units and disposal of waste that may be retrieved.

\section{ACTIVITY:}

Establish programmatic and functional responsibilities for the various programs and establish the lead responsible program.

Establish a formal and controlled integrated program baseline plan/schedule and implement.

ACTIONEE: Westinghouse Hanford, DOE-RL, and DOE-HQ

ADS: Not applicable 
II. Ability to meet committed closure milestones is uncertain.

C. SST program is not integrated.

4. Linkage of SST and DST activities have not been subjected to a systems analysis to optimize program.

NEED:

The SST and OST program will require similar facilities for partitioning and treating waste. If DST waste processing requires new facilities, the facilities need to be capable of handling both wastes.

\section{ACTIVITY:}

Conduct systems engineering studies that evaluate both the SST and DST programs and integrate results.

ACTIONEE: Westinghouse Hanford

ADS: 5175; Waste Management support to be determined. 
II. Ability to meet committed closure milestones is uncertain.

C. SST program is not integrated.

5. Schedule for availability of repositories impacts disposal of DST waste and may impact disposal of SST waste.

NEED:

Planning at the DOE-HQ level needs to include plans and schedule to provide national repository space for disposal of SST high-level waste forms.

Contingency planning needs to be included in the SST System Engineering study to provide for interim storage of waste to be disposed in the repository.

\section{ACTIVITY:}

DOE-HQ develop plans and schedule to provide repository space (or possible interim storage) for disposal of SST high-level waste forms.

Include in the SST System Engineering study planning for potential interim storage of waste to be disposed in the repository.

ACTIONEE: Westinghouse Hanford, DOE-RL, and DOE-HQ

ADS : 5175 
II. Ability to meet committed closure milestones is uncertain.

D. Current 2-year budget cycle is not responsive to evolving reeds.

1. Previous budget targets established 2 years ago (prior to tank safety activity) do not meet current requirements.

NEED:

Need to evolve to a more stable situation.

\section{ACTIVITY:}

As the safety program plans are being prepared and reviewed, budget rieeds for FY 1991 and all future years are being established. Reprogramming requests and ADS revisions are being prepared and reviewed.

ACTIONEE: Westinghouse Hanford and DOE-RL

ADS: Not applicable 
II. Ability to meet committed closure milestones is uncertain.

D. Current 2-year budget cycle is not responsive to evolving needs.

2. Implementation of the SST System Engineering study findings to support the 2018 closure milestone will require supplemental or reprogrammed funding in FY 1992.

Under FY 1992 Environmental Restoration SST ADSS, the planning budget requested was based upon the SST Closure SEIS starting in 1996 and issuing the draft SEIS for public review in FY 2002 (M-09-01). The SST Systems Engineering study (in progress) has identified the need to complete the SEIS earlier to meet the TriParty Agreement 2018 scheduled date for closure of SSTs.

NEED:

Required level of funding is necessary for Environmental Restoration SST ADSs in FY 1992 to prepare the notice of intent for the SST Closure SEIS and to start the SST operable unit ancillary sites characterization. Bringing the SEIS and supporting activities forward in schedule will allow the record of decision to be made and allow necessary time for design, permits, and constructing the closure facilities and to operate and close the SSTs by the 2018 Tri-Party Agreement scheduled date.

Required level of funding is needed for Waste Management to resolve SST safety issues permitting progress on closure.

Required level of funding is needed for Technology Development to advance SST closure and waste disposal technical alternatives to provide information for preparation of the SEIS.

\section{ACTIVITY:}

Provide required funding level in FY 1992. Prepare supplemental or reprogramming requests as required.

ACTIONEE: DOE-RL and DOE-HQ

ADS: $\quad 5175$ 
II. Ability to meet committed closure milestones is uncertain.

E. Until closure plan is prepared and approved, the technical and programmatic baseline (design/construction/operation scope, schedule, cost) cannot be finalized.

1. Characterization of waste and operable units is not complete.

NEED:

SST waste characterization plan has not been approved by Ecology. Characterization effort is still in its early stages with requirements for routine characterization effort still uncertain.

SST waste analytical methods development has not been funded in FY 1991 and FY 1992 (guidance given for FY 1993 ADS planning). Sampling development has received partial funding.

Characterization efforts necessary to satisfy SEIS and Closure technical and schedule requirements need to be defined.

\section{ACTIVITY:}

Complete the SST System Closure/Corrective Action Work Plan and establish level of characterization necessary to satisfy SST SEIS and closure technical and schedule requirements.

Obtain approval from Ecology on the SST Waste Characterization Plan.

Develop necessary sampling and analytical methodologies up-front, increasing efficiency by decreasing worker time in taking samples and in exposure to radiation; and reducing cost by minimizing possible resampling later on in the characterization effort.

ACTIONEE: Westinghouse Hanford, DOE-RL, and DOE-HQ

ADS: $\quad 5175$ and 5176 
II. Ability to meet committed closure milestones is uncertain.

E. Until closure plan is prepared and approved, the technical and programmatic baseline (design/construction/operation scope, schedule, cost) cannot be finalized.

2. Risk assessment is not complete.

\section{NEED :}

Application of just regulator-based requirements for deciding whether SST waste is retrieved and/or left in-place needs to be reviewed and compared. to a risk-based approach for making these decisions. The risk-based approach needs to be developed, the criteria for disposal decisions needs to be defined, and an agreement reached between DOE and the regulators, Ecology, and EPA.

\section{ACTIVITY:}

Develop risk-based approach including application of regulatory equipments for determining criteria for making SST operable unit waste retrieve and/or leave in-place decisions in support of the SST operable unit closures and obtain agreement of the regulators.

ACTIONEE: Westinghouse Hanford, DOE-RL, and DOE-HQ

ADS: 5175 and 5176 
II. Ability to meet committed closure milestones is uncertain.

E. Until closure plan is prepared and approved, the technical and programmatic baseline (design/construction/operation scope, schedule, cost) cannot be finalized.

3. Proposed closure action selection is dependent upon NEPA record of decision.

NEED:

Apply a system engineering approach to identify and to evaluate the technical alternatives in support to the NEPA decision-making process for closure of the SST operable units.

ACTIVITY:

Complete the SST System Engineering study.

Prepare the SST SEIS.

ACTIONEE: Westinghouse Hanford and a "to-be-determined contractor"

ADS: $\quad 5175$ 
II. Ability to meet committed closure milestones is uncertain.

E. Until closure plan is prepared and approved, the technical and programmatic baseline (design/construction/operation scope, schedule, cost) cannot be finalized.

4. Engineering data are incomplete.

NEED:

Complete the conceptual engineering for leading alternatives that provide a basis for planning.

ACTIVITY:

Perform Systems Engineering study.

ACTIONEE: Westinghouse Hanford and DOE-RL

ADS: 5175 
II. Ability to meet committed closure milestones is uncertain.

E. Until closure plan is prepared and approved, the technical and programmatic baseline (design/construction/operation scope, schedule, cost) cannot be finalized.

5. Public participation/review has not been initiated.

NEED:

Meaningful public involvement program is necessary to create a base of awareness and understanding in the general public and interested parties of the SST program. The primary purpose for this program is to build awareness and understanding of SST safety issues and the resolution process, the SST closure process and the process of disposing waste that may be retrieved from the SSTS.

The public involvement program must give the public an opportunity to comment knowledgeably and meaningfully on particular issues and to provide a method for DOE feedback on resolution of issues in areas of public concern.

\section{ACTIVITY:}

Establish a public involvement plan for SST program and implement.

Coordinate the developmerit and implementation of the plan with existing public outreach activities.

ACTIONEE: Westinghouse Hanford, DOE-RL, and DOE-HQ

ADS: 5175, Waste Management to be determined 
II. Ability to meet committed closure milestones is uncertain.

E. Until closure plan is prepared and approved, the technical and programmatic baseline (design/construction/operation scope, schedule, cost) cannot be finalized.

6. Final draft closure $p l$ an has not been prepared and submitted to regulators for approval.

NEED :

Prepare the SST closure plan(s).

ACTIVITY:

Prepare the SST closure plan(s).

ACTIONEE: Westinghouse Hanford

ADS: Not applicable. Beyond the time frame in the current ADS planning. 
II. Ability to meet committed closure milestones is uncertain.

F. Until SST Closure SEIS is prepared, approved, and records of decision issued, SST program cannot restrict options and optimize schedule.

1. Characterization required by HOW-EIS record of decision has not been completed.

NEED:

Need to develop an integrated plan and schedule to sample and characterize the SST waste.

Need to determine scope of characterization for SST waste and ancillary sites within the SST operable units to satisfy the requirements of NEPA.

\section{ACTIVITY:}

Establish scope of characterization effort for the SST operable units to satisfy requirements of NEPA/SEIS.

Prepare a SST program integrated plan and schedule for characterizing the SST operable units.

ACTIONEE: Westinghouse Hanford, DOE-RL, and DOE-HQ

ADS: 5175, Waste Management support to be determined. 
II. Ability to meet committed closure milestones is uncertain.

F. Until SST Closure SEIS is prepared, approved, and records of decision issued, SST program cannot restrict options and optimize schedule.

2. Waste retrieval and treatment technology evaluation required by the HDW-EIS record of decision has not been completed.

\section{NEED:}

Evaluate the potential retrieval and treatment technology options to support disposing of the SST waste.

\section{ACTIVITY:}

Perform a systems engineering study to evaluate retrieval and treatment technology options.

ACTIONEE: Westinghouse Hanford

ADS: $\quad 5175$ 
II. Ability to meet committed closure milestones is uncertain.

F. Until SST Closure SEIS is prepared, approved, and records of decision issued, SST program cannot restrict options and optimize schedule.

3. Waste destruction, stabilization technology, and burial performance required by the HDW-EIS record of decision has not been completed.

NEED:

Determine the level of engineering data necessary for evaluation of potential waste destruction, stabilization technology, and burial performance needed to satisfy HDW-EIS record of decision and to support the decision-making process of the SST Closure SEIS record of decision.

The HOW-EIS record of decision requires barrier performance be demonstrated by both instrumented field tests and modeling; the need and methods to improve the stability of the waste form need to be determined, and destruction or stabilization alternatives for hazardous constituents evaluated.

\section{ACTIVITY:}

Establish criteria for the SEIS in conjunction with pre-scoping activities.

ACTIONEE: Westinghouse Hanford, DOE-RL, and DOE-HQ

ADS: 5175 
II. Ability to meet committed closure milestones is uncertain.

F. Until SST Closure SEIS is prepared, approved, and records of decision issued, SST program cannot restrict options and optimize schedule.

4. Interface with tank safety NEPA process has not been established.

NEED:

Need to determine the scope of the tank safety SEIS document. A determination as to whether the tank safety SEIS only covers the consequence analys is or other related areas (i.e., corrective action facilities). A determination is required regarding consolidation of tank safety SEIS and Closure SEIS scope.

ACTIVITY:

Begin pre-scoping activities (e.g., conduct an evaluation of the alternatives and integrate the recommendations with SST engineering study) for the tank safety NEPA documentation.

ACTIONEE: Westinghouse Hanford, DOE-RL; and DOE-HQ

ADS: 5175 and 9855 
II. Ability to meet committed closure milestones is uncertain.

F. Until SST Closure SEIS is prepared, approved, and records of decision issued, SST program cannot restrict options and optimize schedule.

5. SST Closure SEIS review and approval schedule is not internally controlled.

NEED:

DOE-RL and DOE-HQ need to provide timely review and action on NEPA documentation.

\section{ACTIVITY:}

Streamline the approval process by placing the decision-making authority as close to those doing the work as possible.

ACTIONEE: DOE-RL and DOE-HQ

ADS: Not applicable 
II. Ability to meet committed closure milestones is uncertain.

F. Until SST Closure SEIS is prepared, approved, and records of decision issued, SST program cannot restrict options and optimize schedule.

6. Corrective action under $3004(u)$ may be unable to support an accelerated SST Closure SEIS.

NEED:

The amount and type of characterization activities supporting the SEIS have not been defined. The current characterization support designation of the waste to meet RCRA requirements.

\section{ACTIVITY:}

Carry a range of alternatives on the SEIS that support a range of characterization results. Characterization will ultimately show a need for several kinds of closure actions to deal with the variability in the soil contamination.

ACTIONEE: Westinghouse Hanford

ADS: $\quad 5175$ 
II. Ability to meet committed closure milestones is uncertain.

F. Until SST Closure SEIS is prepared, approved, and records of decision issued, SST program cannot restrict options and optimize schedule.

7. Design, permitting, and construction of the retrieval option does not support the 2018 committed milestone.

\section{NEED :}

The technical decision basis needs to be strengthened for evaluating acceleration of characterization technology evaluation and Closure SEIS preparation. Enhancement of the permitting schedule will require a close working relationship with the State and EPA. Schedule impact to the-Tri-Party Agreement will be resolved through the established process.

\section{ACTIVITY:}

Determine level of engineering data required to support Closure SEIS preparation. Conduct systems engineering evaluation of retrieval alternatives and schedules. Begin regulator participation for establishing permitting strategy.

ACTIONEE: Westinghouse Hanford, DOE-RL, and DOE-HQ

\section{ADS: 5175}


DOE/RL- $90-48$

Predecisional Draft

$4 / 5 / 91$

This page intentionally left blank. 
$D O E / R L-90-48$

Predecisional Draft

$4 / 5 / 91$

7.3 ISSUE III - NEPA PATHWAYS HAVE NOT BEEN DEFINED 
This page intentionally left blank. 


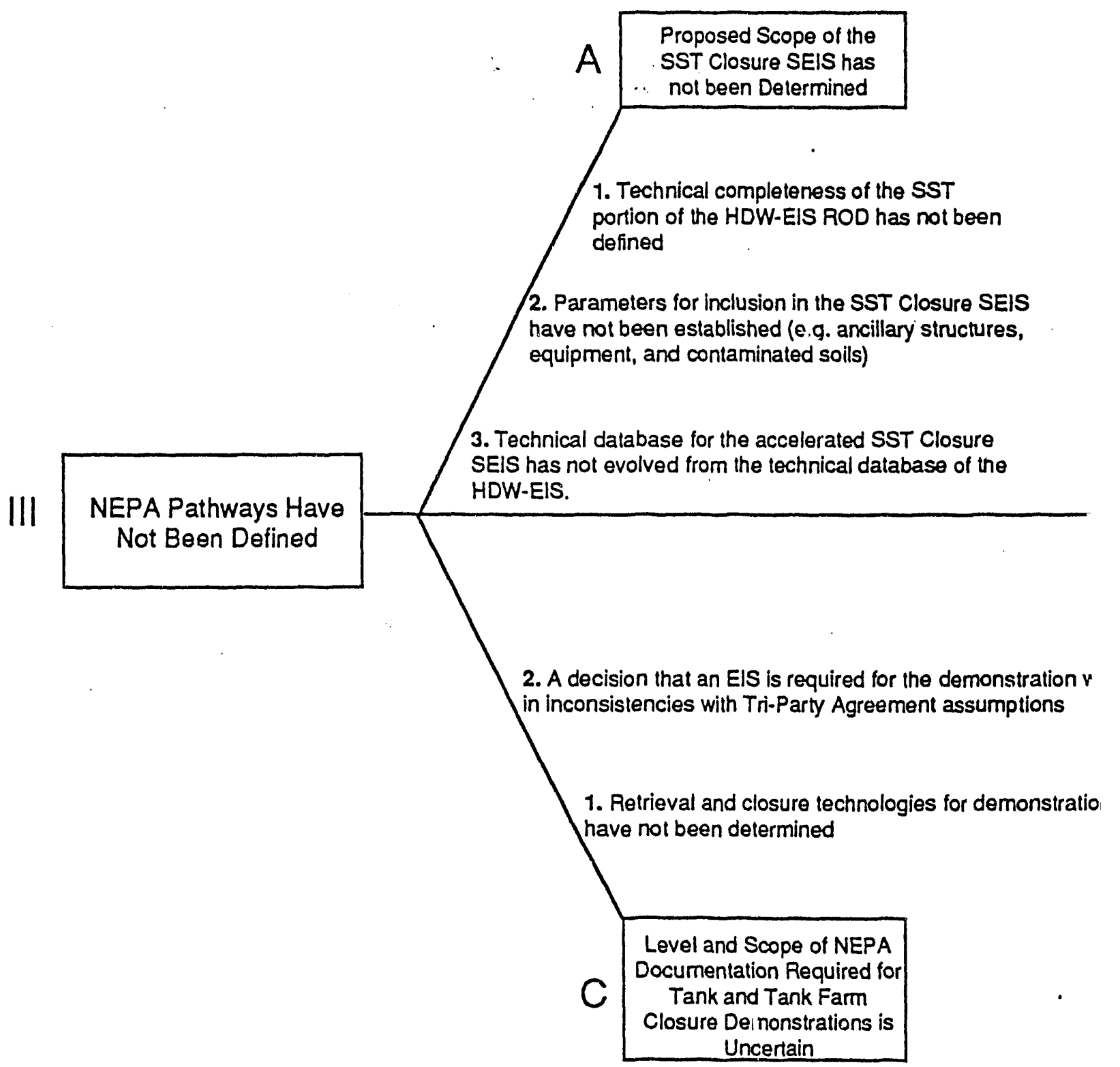


DOE/RL $-90-48$

Predecisional Draft

$4 / 5 / 91$

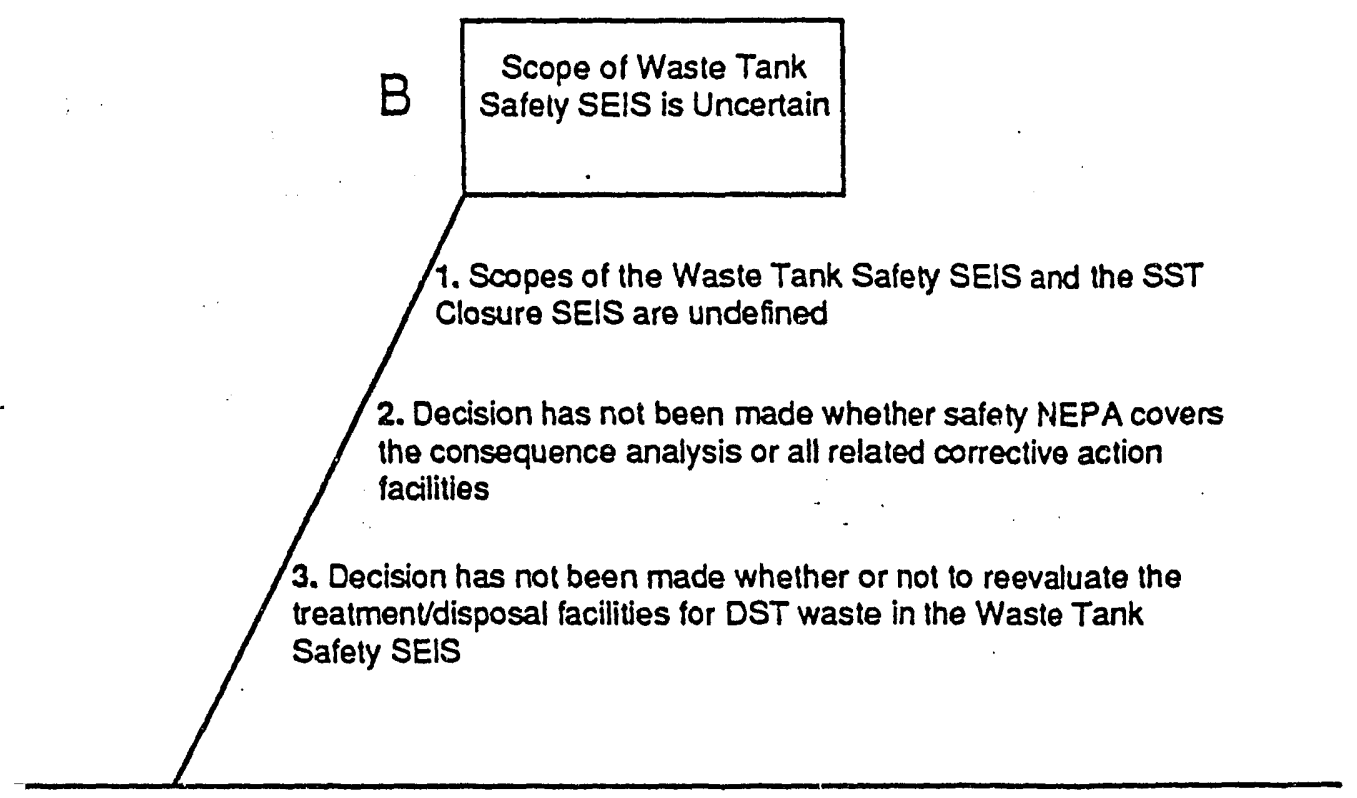

suld result

s

Figure 7-3. Issue III - NEPA Pathways Have not Been Defined. 
III. NEPA pathways have not been defined.

A. Proposed scope of the SST Closure SEIS has not been determined.

1. Technical completeness of the SST portions of the HOW-EIS record of decision has not been defined.

\section{NEED:}

DOE needs to determine if the guidelines of the HOW-EIS record of decision have been satisfied for determining adequate characterization, barrier performance, waste form stability, etc.

\section{ACTIVITY:}

Make decisions for above.

ACTIONEE: DOE-RL and DOE-HQ

ADS: Not applicable 
III. NEPA pathways have not been defined.

A. Proposed scope of the SST Closure SEIS has not been determined.

2. Parameters for inclusion in the SST Closure SEIS have not been established (e.g., ancillary structures, equipment, and contaminated soils)

NEED:

The scope of the accelerated SST Closure SEIS must be determined.

ACTIVITY:

Begin pre-scoping activities.

ACTIONEE: Westinghouse Hanford, DOE-RL, and DOE-HQ

ADS: 5175 
DOE/RL- $90-48$

Predecisional Draft

$4 / 5 / 91$

III. NEPA pathways have not been defined.

A. Proposed scope of the SST Closure SEIS has not been determined.

3. Technical database for the accelerated SST Closure SEIS has not evolved from the technical database of the HDW-EIS.

\section{NEED:}

Need the following: data technology development, waste characterization, waste from stabilization, barrier performance, and studies on pretreatment, processing, etc.

\section{ACTIVITY:}

Need to accelerate the above processes.

ACTIONEE: Westinghouse Hanford

ADS: 5175,5176 , and 5178 
III. NEPA pathways have not been defined.

B. Scope of Waste Tank Safety SEIS is uncertain.

1. Scopes of the Waste Tank Safety SEIS and the SST Closure SEIS are undefined.

NEED:

Need to determine the scope of the Waste Tank Safety SEIS.

ACTIVITY:

Begin pre-scoping activities. DOE-HQ will finalize scope and issue notice of intent.

ACTIONEE: Westinghouse Hanford, DOE-RL, and DOE-HQ

ADS: 9854 and 9855 
III. NEPA pathways have not been defined.

B. Scope of Waste Tank Safety SEIS is uncertain.

2. Decision has not been made whether safety NEPA covers the consequence analysis or all related corrective action facilities.

NEED:

Need to determine the scope of the safety NEPA document.

\section{ACTIVITY:}

Begin pre-scoping activities. DOE-HQ will finalize sçope and issue a notice of intent.

ACTIONEE: Westinghouse Hanford, DOE-RL, and DOE-HQ

ADS: 9854 and 9855 
III. NEPA pathways have not been defined.

B. Scope of Waste Tank Safety SEIS is uncertain.

3. Decision has not been made whether or not to reevaluate the treatment/disposal facilities for DST waste in the Waste Tank Safety SEIS.

\section{NEED:}

The HOW-EIS defines treatment and disposal facilities of the 28 DSTS.

However, since five of the DSTs are watch list tanks, the SST SEIS may also propose treatment and disposal processes for the OSTS. These watch list tanks have to comply with the requirements of the Safety Measures Law. If complying with these new requirements mandates the DOE to deviate from the record of decision for the HOW-EIS, the course contract personnel have to follow need to be determined.

\section{ACTIVITY:}

The DOE needs to decide within the time frame of the Safety Measures Law what takes precedence the existing HOW-EIS or the Waste Tank Safety SEIS.

ACTIONEE: Westinghouse Hanford, DOE-RL, and DOE-HQ

ADS: To be determined 


$$
\text { DOE/RL }-90-48
$$

III. NEPA pathways have not been defined.

C. Level and scope of NEPA documentation required for tank and tank farm closure demonstrations is uncertain.

1. Retrieval and closure technologies for demonstrations have not been determined.

\section{NEED:}

Need to determine levels of NEPA documentation required for the tank demonstration and the tank farm closure demonstration.

\section{ACTIVITY:}

Prepare an action description memorandum for these activities or DOE-4.4Q must determine the appropriate level of NEPA documentation.

ACTIONEE: Westinghouse Hanford, DOE-RL, and DOE-HQ

\section{ADS: 5175}


III. NEPA pathways have not been defined.

C. Level and scope of NEPA documentation required for tank and tank farm closure demonstrations is uncertain.

2. A decision that an EIS is required foi the demonstration would result in inconsistencies with Tri-Party Agreement assumptions.

\section{NEED :}

The level of NEPA documentation required, the impacts to the closure schedule resulting from the NEPA process, and the method for implementation of SEPA must be determined.

\section{ACTIVITY:}

The above decisions and determinations must be made either through preparation of action description memorandums for DOE-HQ decision or direct decision by DOE-HQ.

ACTIONEE: Westinghouse Hanford, DOE-RL, and DOE-HQ

ADS: 5176,5175 , and 5178 
DOE/RL- $90-48$

Predecisional Draft

$4 / 5 / 91$

7.4 ISSUE IV - REGULATORY CLOSURE REQUIREMENTS (STATE, EPA, DOE, NRC) DO NOT AGREE WITH HDW-EIS RECORD

OF DECISION RISK-BASED APPROACH 
DOE/RL- $90-48$

Predecisional Draft

$4 / 5 / 91$

This page intentionally left blank. 


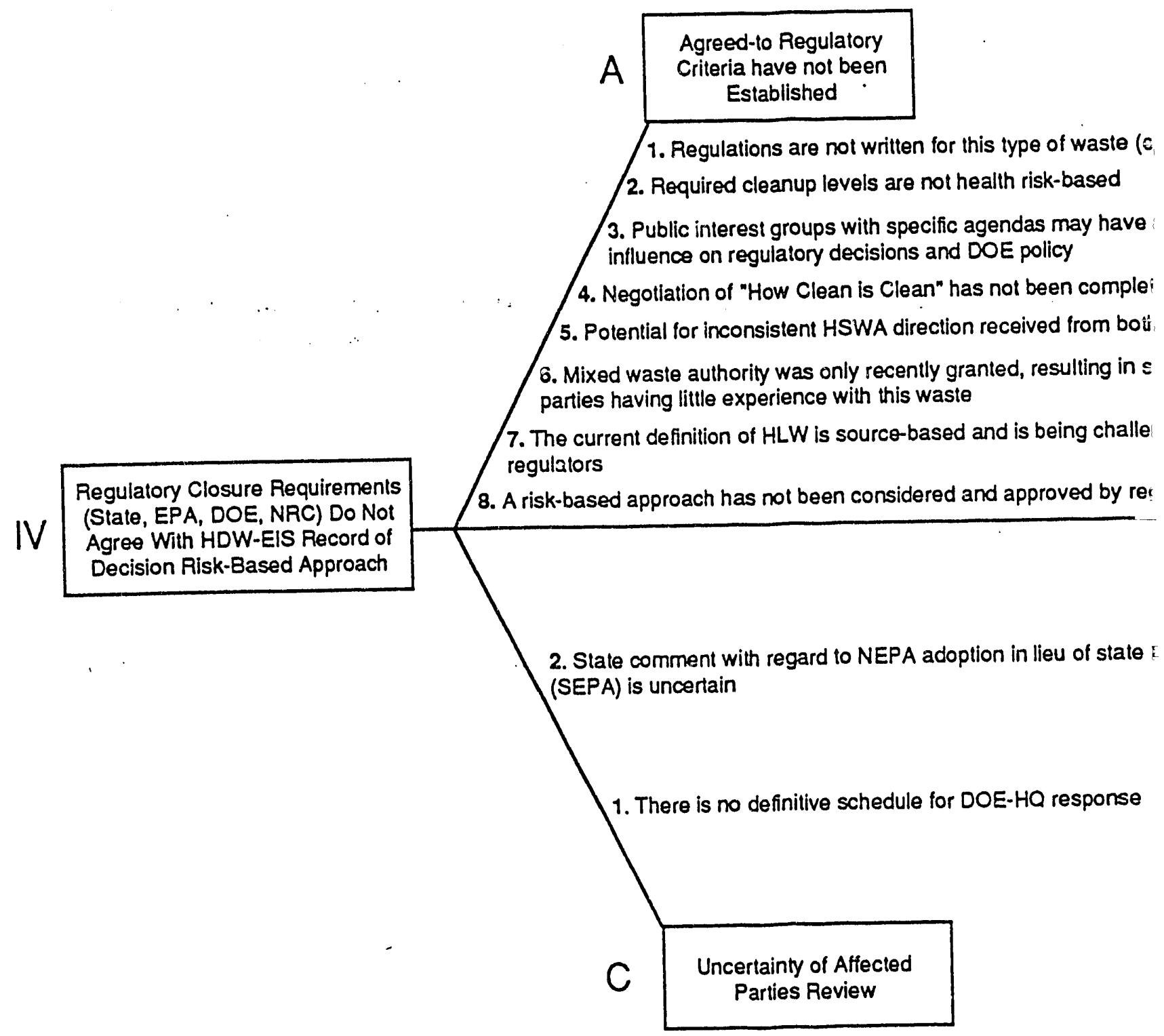


DOE/RL $-90-48$

Predecisional Draft

$4 / 5 / 91$

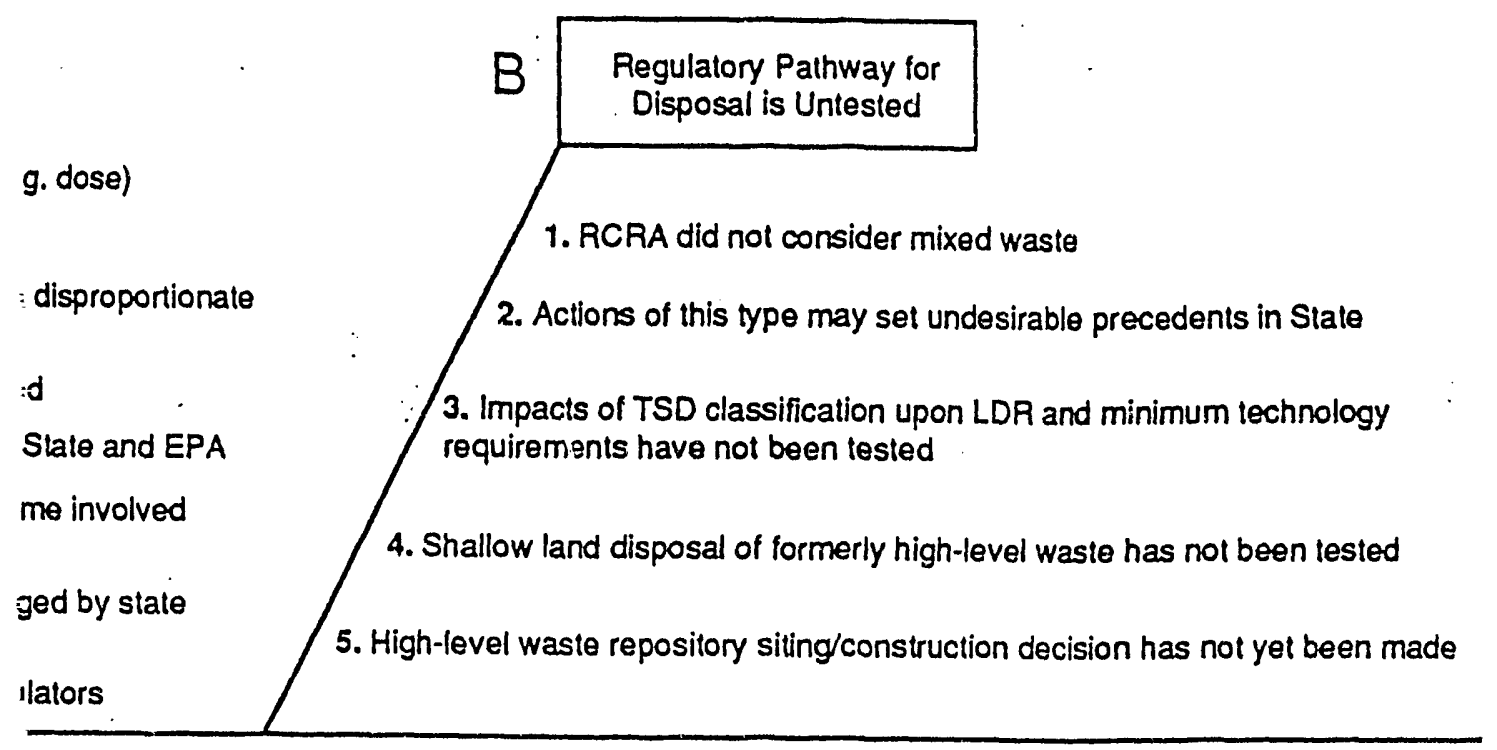

is

Figure 7-4. Issue IV - Regulatory Closure Requirements (State, EPA, DOE, NRC) do not Agree with HDW-EIS Record of Decision Risk-Based Approach. 
IV. Regulatory closure requirements (State, EPA, DOE, NRC) do not agree with HDW-EIS record of decision risk-based approach.

A. Agreed-to regulatory criteria have not been established.

1. Regulations are not written for this type of waste (e.g., dose).

\section{NEED:}

Variances from the "remove or decontaminate" requirements of WAC 173-303-640 may be required.

Regulatory changes may be required to address the above closure requirements (i.e., develop statutory changes that allow treatment and storage tanks to be closed as landfills).

Certain RCRA actions may be inconsistent with AEA requirements and modified procedures will need to be- agreed upon.

\section{ACTIVITY:}

Continue waste characterization, technology development, and perforilance assessment work to support negotiations and/or potential negotiations. Continue National Academy of Science contacts and propose appropriate legislation.

ACTIONEE: Westinghouse Hanford, DOE-RL, and DOE-HQ

\section{ADS: 5175}


IV. Regulatory closure requirements (State, EPA, DOE, NRC) do not agree with HDW-EIS record of decision risk-based approach.

A. Agreed-to regulatory criteria have not been established.

2. Required cleanup levels are not health risk-based.

NEED:

Negotiation of cleanup levels based upon health or environmental risk-based criteria rather than analytical detection limits.

ACTIVITY:

Negotiate with EPA and Ecology to establish appropriate cleanup levels.

ACTIONEE: Westinghouse Hanford and DOE-RL

ADS : 5175 
IV. Regulatory closure requirements (State, EPA, DOE, NRC) do not agree with HDW-EIS record of decision risk-based approach.

A. Agreed-to regulatory criteria have not been established.

3. Public interest groups with specific agendas may have a disproportionate influence on regulatory decisions and DOE policy.

NEED:

Develop data from waste characterization, retrieval technology, and the performance assessment for technical input to the regulators and the public.

\section{ACTIVITY:}

Continue waste sampling, engineering studies and performance assessment work. Continue to schedule public meetings for issue discussions.

ACTIONEE: Westinghouse Hanford, DOE-RL, and DOE-HQ

ADS: 5008,5175 , and 9123 
IV. Regulatory closure requirements (State, EPA, DOE, NRC) do not agree with HOW-EIS record of decision risk-based approach.

A. Agreed-to regulatory criteria have not been established.

4. Negotiation of "How Clean is Clean" has not been completed.

NEED:

Data are required to support a deviation/variance or if excessive worker exposure and/or technical infeasibilities exist that prevent waste removal to background for listed dangerous wastes or characteristic wastes, as specified in current State regulations. Alternatively, data may be used to negotiate with State regarding use of health based, rather than background, standards. Finally, data are needed to facilitate negotiations with EPA regarding cleanup levels for radionuclides.

\section{ACTIVITY:}

Continued technology development, waste characterization, and performance assessment work.

ACTIONEE: Westinghouse Hanford, DOE-RL, and DOE-HQ

ADS: 5175 
IV. Regulatory closure requirements (State, EPA, DOE, NRC) do not agree with HDW-EIS record of decision risk-based approach.

A. Agreed-to regulatory criteria have not been established.

5. Potential for inconsistent Hazardous and Solid Waste Amendments direction received from both State and EPA.

NEED:

Negotiations with EPA and State to establish consistent direction. If the State takes interpretations that are beyond the scope of RCRA, a determination from DOE-HQ may be warranted on the possibility of asserting a sovereign immunity defense from state only requirements.

\section{ACTIVITY:}

Negotiate with EPA and the State to establish consistent and logical requirements.

ACTIONEE: Westinghouse Haıford, DOE-RL, and DOE-HQ

ADS: 5175 
IV. Regulatory closure requirements (State, EPA, DOE, NRC) do not agree with HOW-EIS record of decision risk-based approach.

A. Agreed-to regulatory criteria have not been established.

6. Mixed waste authority was only recently granted, resulting in some involved parties having little experience with this waste.

NEED:

Continued waste characterization, technology development to support various possibilities including development of new regulation under RCRA for mixed waste statutory amendments to RCRA during reauthorization in 1992, rulemaking petitions.

\section{ACTIVITY:}

Continued waste characterization and technology development.

ACTIONEE: Westinghouse Hanford, DOE-RL, and DOE-HQ

ADS: 5175 and 5008 
IV. Regulatory closure requirements (State, EPA, DOE, NRC) do not agree with HOW-EIS record of de:ision risk-based approach.

A. Agreed-to regulatory criteria have not been established.

7. The current definition of high-level waste is source-based and is being challenged by state regulators.

\section{NEED:}

(1) Waste characterization data are needed.

(2) Discuss with the NRC when data are available.

(3) If defined as high-level waste, SST waste which remains in place will be subject to NRC licensing for shallow land burial of high-level waste.

\section{ACTIVITY:}

The NRC determines whether past processing is sufficient for some wastes to be non-high-level waste. The determination will be made tank-by-tank when characterization data are available.

Continued waste characterization develop a basis for discussion with the NRC.

ACTIONEE: Westinghouse Hanford, DOE-RL, and DOE-HQ

ADS: 5175 
IV. Regulatory closure requirements (State, EPA, DOE, NRC) do not agree with HDW-EIS record of decision risk-based approach.

A. Agreed-to regulatory criteria have not been established.

8. A risk-based approach has not been considered and approved by regulators.

NEED:

(1) Data from waste characterization, worker exposure, and performance assessment results to support a risk-based approach.

(2) Continued negotiations with State and EPA to establish acceptable risk level.

(3) Regulatory changes in the form of rulemaking petitions or RCRA reauthorization may be necessary. Alternatively, DOE-HQ evaluation of a potential AEA inconsistency determination may be warranted.

\section{ACTIVITY:}

Continued waste characterization, technology development, and performance assessment work. Continue National Academy of Sciences contacts and propose appropriate legislation.

ACTIONEE: Westinghouse Hanford, DOE-RL, and DOE-HQ

ADS: 5175 
IV. Regulatory closure requirements (State, EPA, DOE, NRC) do not agree with HOW-EIS record of decision risk-based approach.

B. Regulatory pathway for disposal is untested.

1. RCRA did not consider mixed waste.

NEED:

Variances from RCRA regulations may be required. Alternatively, DOE-HQ may need to determine if an AEA exemption is required/justified for SSTS.

\section{ACTIVITY:}

Information related to waste characterization and technology development is needed to support the final disposal options.

ACTIONEE: Westinghouse Hanford, DOE-RL, and DOE-HQ

ADS: 5175 and 5176 
IV. Regulatory closure requirements (State, EPA, DOE, NRC) do not agree with HDW-EIS record of decision risk-based approach.

B. Regulatory pathway for disposal is untested.

2. Actions of this type may set undesirable precedents in State.

NEED:

Information from waste characterization, technology development, and performance assessment will be needed to support the landfill disposal. option for SST waste.

\section{ACTIVITY:}

Continued waste characterization, technology development, and performance assessment work.

ACTIONEE: Westinghouse Hanford

ADS: 5175,5176 , and 5178 
DOE/RL- $90-48$

Predecisional Draft

$4 / 5 / 91$

IV. Regulatory closure requirements (State, EPA, DOE, NRC) do not agree with HDW-EIS record of decision risk-based approach.

B. Regulatory pathway for disposal is untested.

3. Impacts of TSD classification upon LDR and minimum technology requirements have not been tested.

NEED:

(1) A determination is needed regarding the applicability of LDR and minimum technology requirements to SST waste that may be disposed via in situ treatment and disposal.

(2) If waste exists above LDR concentrations, it may be necessary to develop a treatability variance.

\section{ACTIVITY:}

Waste characterization data are needed to support classification.

ACTIONEE: Westinghouse Hanford, DOE-RL, OOE-HQ, EPA, and Ecology

ADS: 5178 
IV. Regulatory closure requirements (State, EPA, DOE, NRC) do not agree with HDW-EIS record of decision risk-based approach.

B. Regulatory pathway for disposal is untested.

4. Shallow land disposal of formerly high-level wast a has not been tested.

NEED:

(1) A determination of whether or not SST waste is high-level waste is needed from the NRC. Negotiations may be necessary to establish a highlevel waste definition that is based upon activity levels of strontium and cesium.

(2) Guidance from the NRC regarding shallow land disposal is needed for licensing purposes.

\section{ACTIVITY:}

Continued waste characterization is required to determine if DOE-HQ should pursue establishment of a new definition of high-level waste based on. activity.

ACTIONEE: Westinghouse Hanford, DOE-RL, DOE-HQ, and NRC

ADS: 5178 
IV. Regulatory closure requirements (State, EPA, DOE, NRC) do not agree with HDW-EIS record of decision risk-based approach.

B. Regulatory pathway for disposal is untested.

5. High-level waste repository siting/construction decision has not yet been made.

NEED :

Need to determine where and when high-level waste will be disposed. Current planning is based on having a repository available on January 1, 2010.

ACTIVITY:

Proceed with repository planning and approval of repository.

ACTIONEE: DOE-HQ

ADS: To be determined 


\section{DOE/RL $-90-48$ \\ Predecisional Draft}

IV. Regulatory closure requirements (State, EPA, DOE, NRC) do not agree with HDW-EIS record of decision risk-based approach.

C. Uncertainty of affected parties review.

1. There is no definitive schedule for DOE-HQ response.

NEED :

DOE-HQ has not committed to schedules on responses to NEPA documentation.

ACTIVITY:

DOE-HQ needs to prepare and meet schedules for responses and decisions on NEPA documentation.

ACTIONEE: DOE-HQ

ADS: Not applicable 
IV. Regulatory closure requirements (State, EPA, DOE, NRC) do not agree with HOW-EIS record of decision risk-based approach.

C. Uncertainty of affected parties review.

2. State comment with regard to NEPA adoption in lieu of state EIS (SEPA) is uncertain.

NEED:

State comments on the scope of the SST Closure SEIS need to be carefully considered to ensure State adoption of SST Closure SEIS.

\section{ACTIVITY:}

Begin negotiations with Ecology to incorporate the State's comments and concerns over the scope of the accelerated SST Closure SEIS.

ACTIONEE: Westinghouse Hanford, DOE-RL, and DOE-HQ

ADS: $\quad 5175$ 


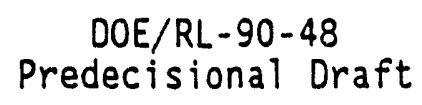

$4 / 5 / 91$

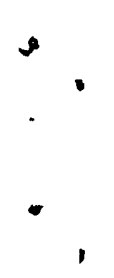

This page intentionally left blank. 


\subsection{ISSUE $V$ - TECHNOLOGY DEVELOPMENT PROCESS IS STILL EVOLVING}


This page intentionally left blank. 


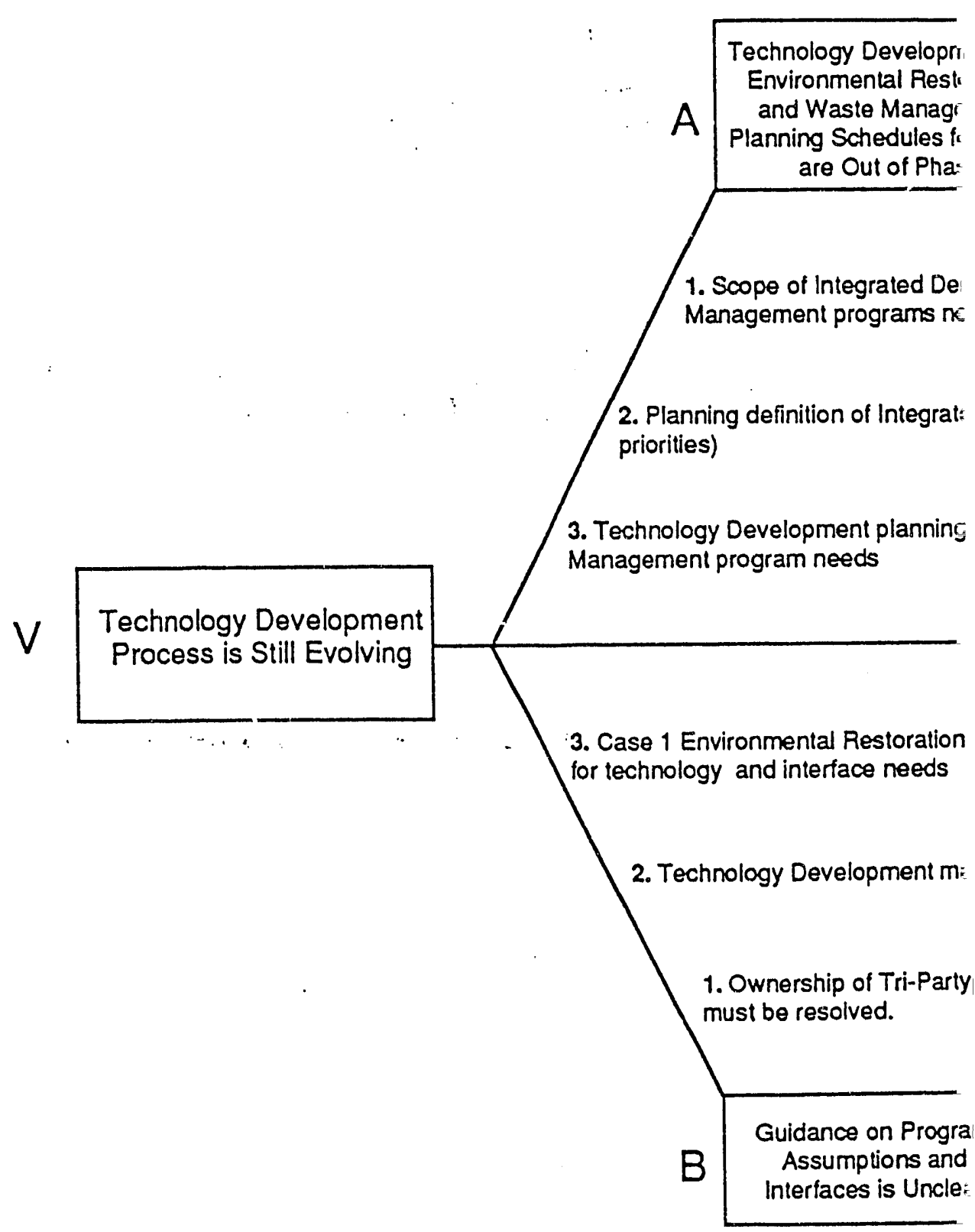


$D O E / R L-90-48$

Predecisional Draft

$4 / 5 / 91$

ent and

ration

ment

r Tanks

: 8

nonstration arid Environmental Restoration and Waste

ed to be integrated (national needs vs. site specific)

d Demonstrations require site input from programs (needs and

process may lag site Environmental Restoration and Waste

program comments requested ADS from Environmental Restoration

$y$ not satisfy all tank technology developrnent needs

Agreement milestones versus responsibilities for basis of milestones

Figure 7-5. Issue $V$ - Technology Development Process is Still Evolving. 
V. Technology development process is still evolving.

A. Technology Development, Environmental Restoration, and Waste Management planning schedules for tanks are out of phase.

1. Scope of Integrated Demonstration, Environmental Restoration, and Waste Management Programs need to be integrated (national needs versus site-specific).

NEED:

The scope of the Integrated Demonstrations in relation to the Environmental Restoration and Waste Management Programs must be clearly defined. The extent to which the integrated demonstrations will address site-specific issues must be clearly defined and communicated.

\section{ACTIVITY:}

Environmental Restoration and Waste Management will be involved in underground storage tank-integrated demonstration planning. Results from underground storage tank-integrated demonstration planning will be integrated with Environmental Restoration and Waste Management programs.

ACTIONEE: Westinghouse Hanford, DOE-RL, and DOE-HQ

ADS : 5179 
V. Technology development process is still evolving.

A. Technology Development, Environmental Restoration, and Waste Management planning schedules for tanks are out of phase.

2. Planning definition of Integrated Demonstrations require site input from programs (needs and priorities).

NEED:

A formal mechanism must be developed for the input and consideration of programmatic needs and priorities into the integrated demonstration planning. This process must be phased to support programmatic planning needs.

\section{ACTIVITY:}

The Roadmap will identify the SST program technology needs. Incorporation of the needs into the integrated demonstration planning is not a specially identified activity in the Roadmaps process. A "Needs Identification" document along with a feedback mechanism to the programs must be incorporated.

ACTIONEE: Westinghouse Hanford, DOE-RL, and DOE-HQ

ADS: Not applicable 
V. Technology development process is still evolving.

A. Technology Development, Environmental Restoration, and Waste Management planning schedules for tanks are out of phase.

3. Technology development planning process may lag site Environmental Restoration and Waste Management Program needs.

NEED:

A decision is required on how the short-term technology needs of the Environmental Restoration and Waste Management Program will be meet. Either the preparation of integrated demonstration schedule must be accelerated, or the technology needs (not addressed in the Integrated Demonstration schedule) must be met with programmatic funding.

\section{ACTIVITY:}

Develop a Westinghouse Hanford position for approval by DOE-RL and DOE-HQ.

ACTIONEE: Westinghouse Hanford, DOE-RL, and DOE-HQ

ADS: Not applicable 
V. Technology development process is still evolving.

B. Guidance on program assumptions and interfaces is unclear.

1. Ownership of Tri-Party Agreement milestones versus responsibilities for basis of milestones must be resolved.

NEED:

To satisfy programmatic concerns of meeting Tri-Party Agreement milestones, the Technology Development Program must assume ownership of technology development activities that support milestones where appropriate. "Ownership" means either supporting the milestone or developing the technical justification for renegotiation.

\section{ACTIVITY:}

Technology Development plans must ensure support for the Tri-Party Agreement milestone.

ACTIONEE: Westinghouse Hanford, DOE-RL, and DOE-HQ

ADS: Not applicable 
V. Technology development process is still evolving.

B. Guidance on program assumptions and interfaces is unclear.

2. Technology development may not satisfy all tank technology development needs.

\section{NEED:}

Timely planning and feedback is required to meet Tri-Party Agreement milestones.

\section{ACTIVITY:}

Expeditiously proceed with the integrated demonstration needs, analysis, and planning.

ACTIONEE: Westinghouse Hanford and DOE-RL

ADS: Not applicable 
V. Technology development process is still evolving.

B. Guidance on program assumptions and interfaces is unclear.

3. Case 1 Environmental Restoration Program comments requested ADSs from Environmental Restoration for technology and interface needs.

NEED:

A Westinghouse Hanford position must be developed on how funding for environmental restoration technology needs will be addressed during the Underçround Storage Tank Integrated Demonstration Program.

ACTIVITY:

Develop a position paper for DOE-RL and DOE-HQ approval.

ACTIONEE: Westinghouse Hanford, DOE-RL, and DOE-HQ

ADS: 5179 
DOE/RL-90-48

Predecisional Draft

$4 / 5 / 91$

This page intentionally left blank. 
Issues Res

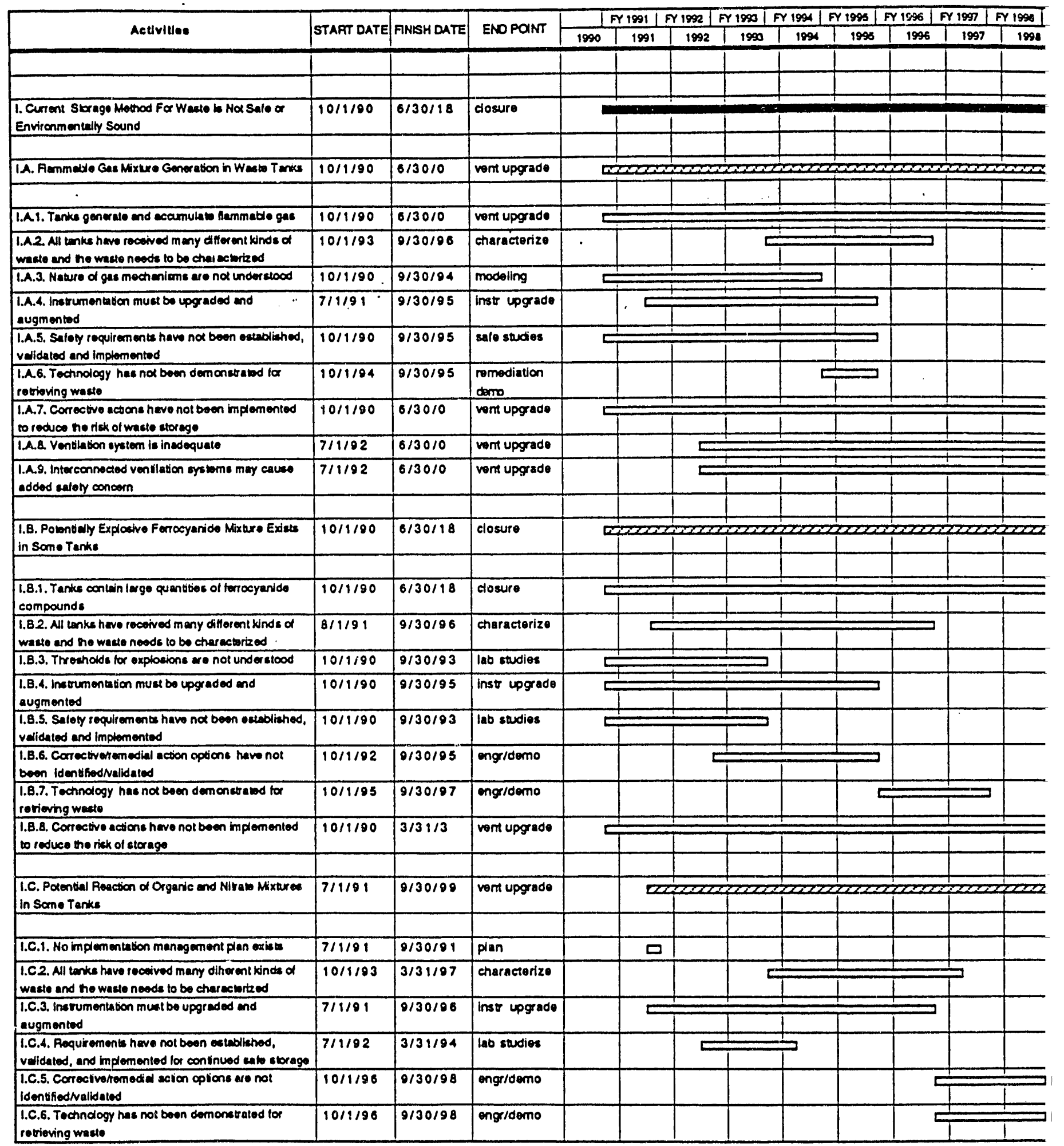


Ilution Schedule

DOE/RL-90-48

Predecisional Draft

$4 / 5 / 91$

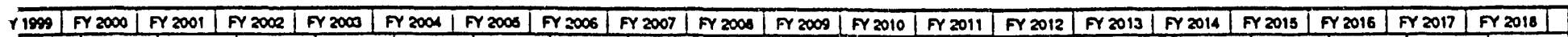

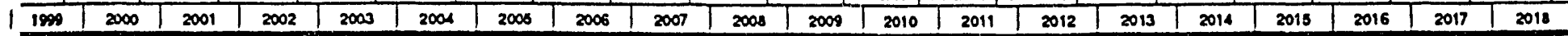
\begin{tabular}{|l|l|l|l|l|l|l|l|l|l|l|l|l|l|l|l|l|l|l|}
\hline & & & & & & & & & & & & & & & & & & \\
\hline
\end{tabular}

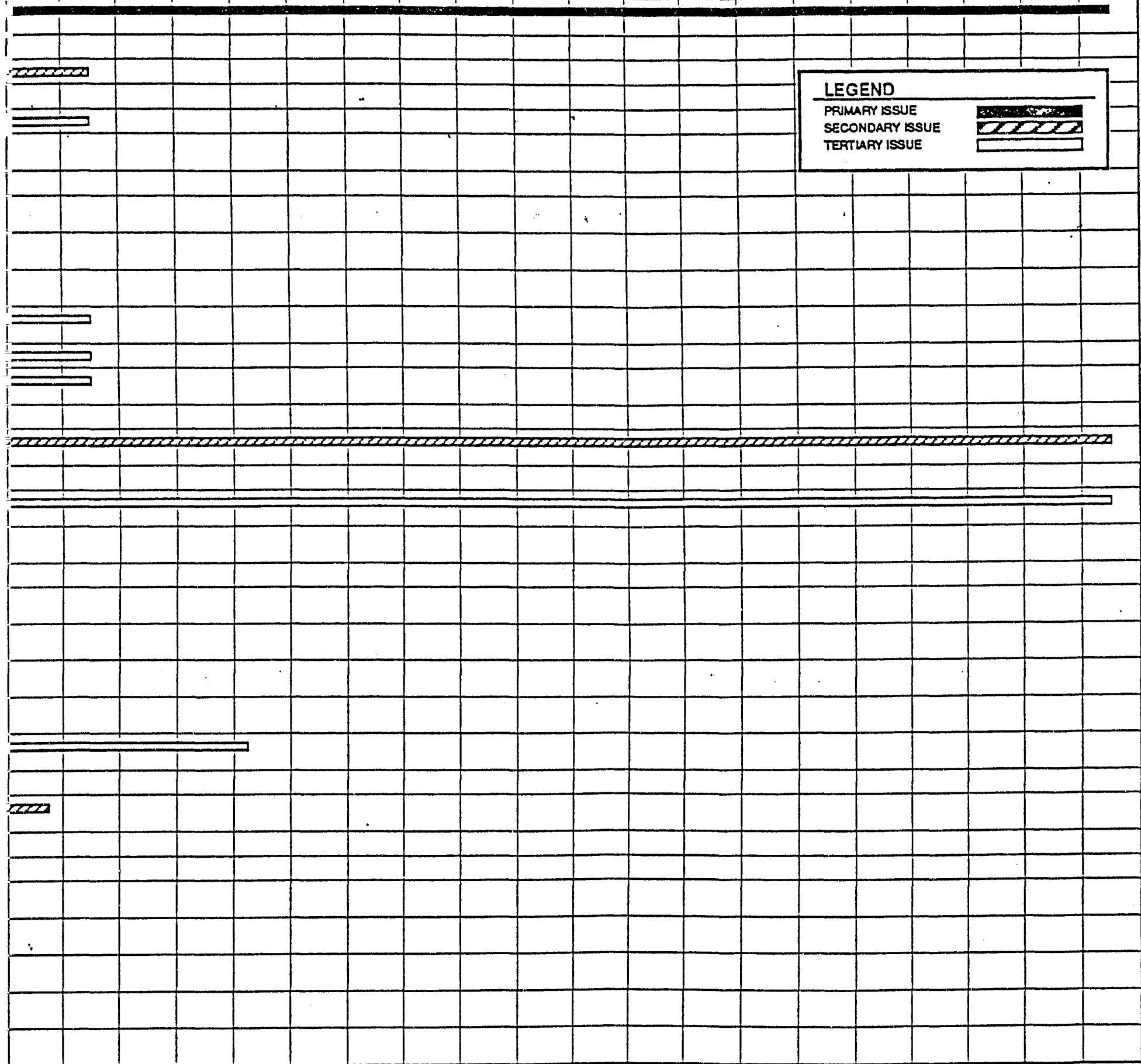


Issues Res

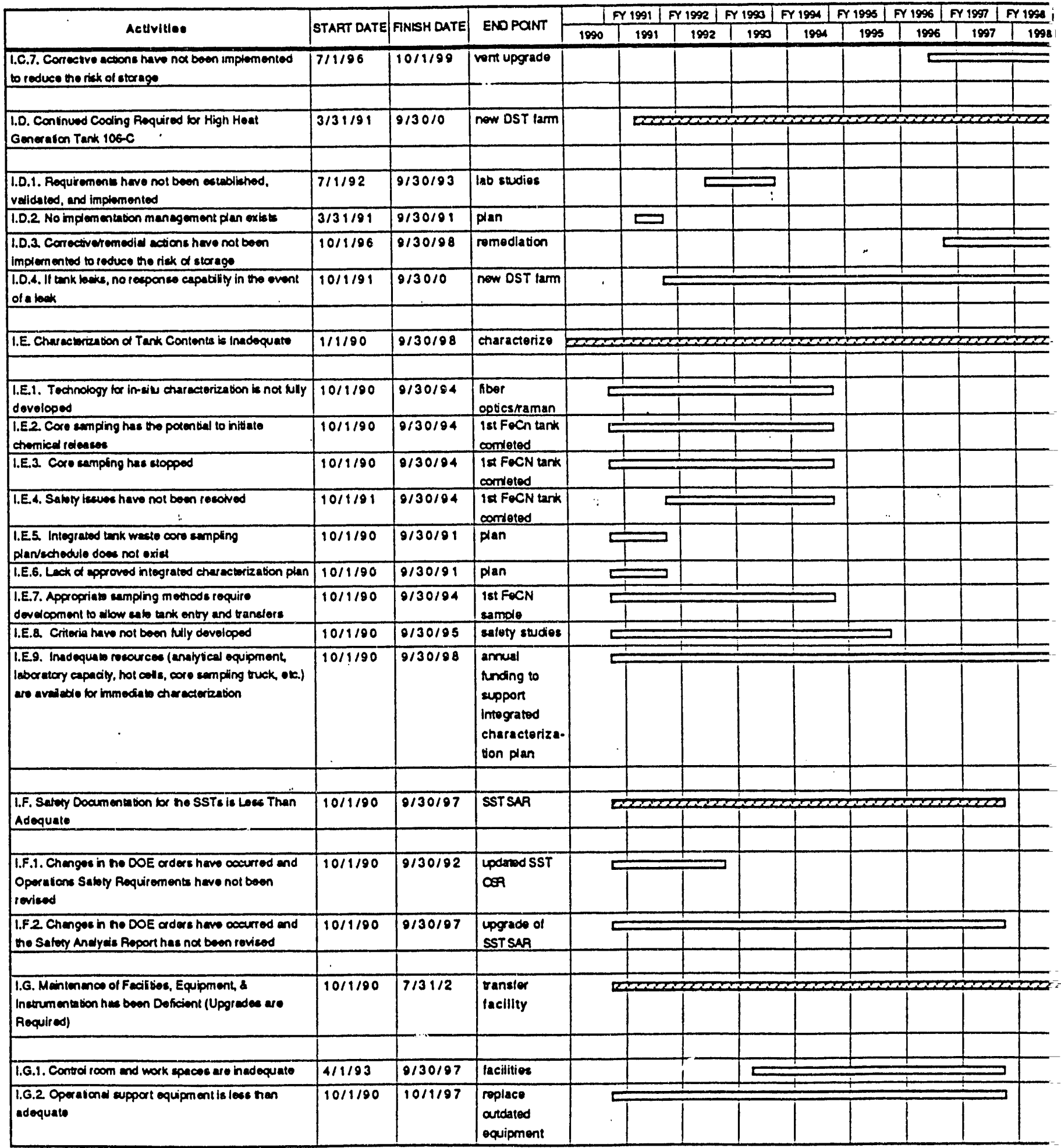


DOE/RL- $90-48$

Predecisional Draft $4 / 5 / 91$

olution Schedule

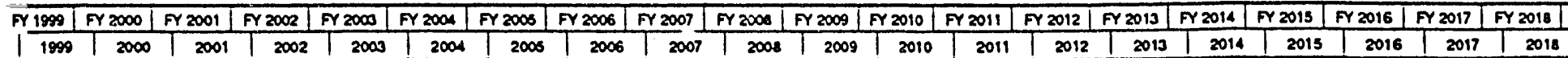

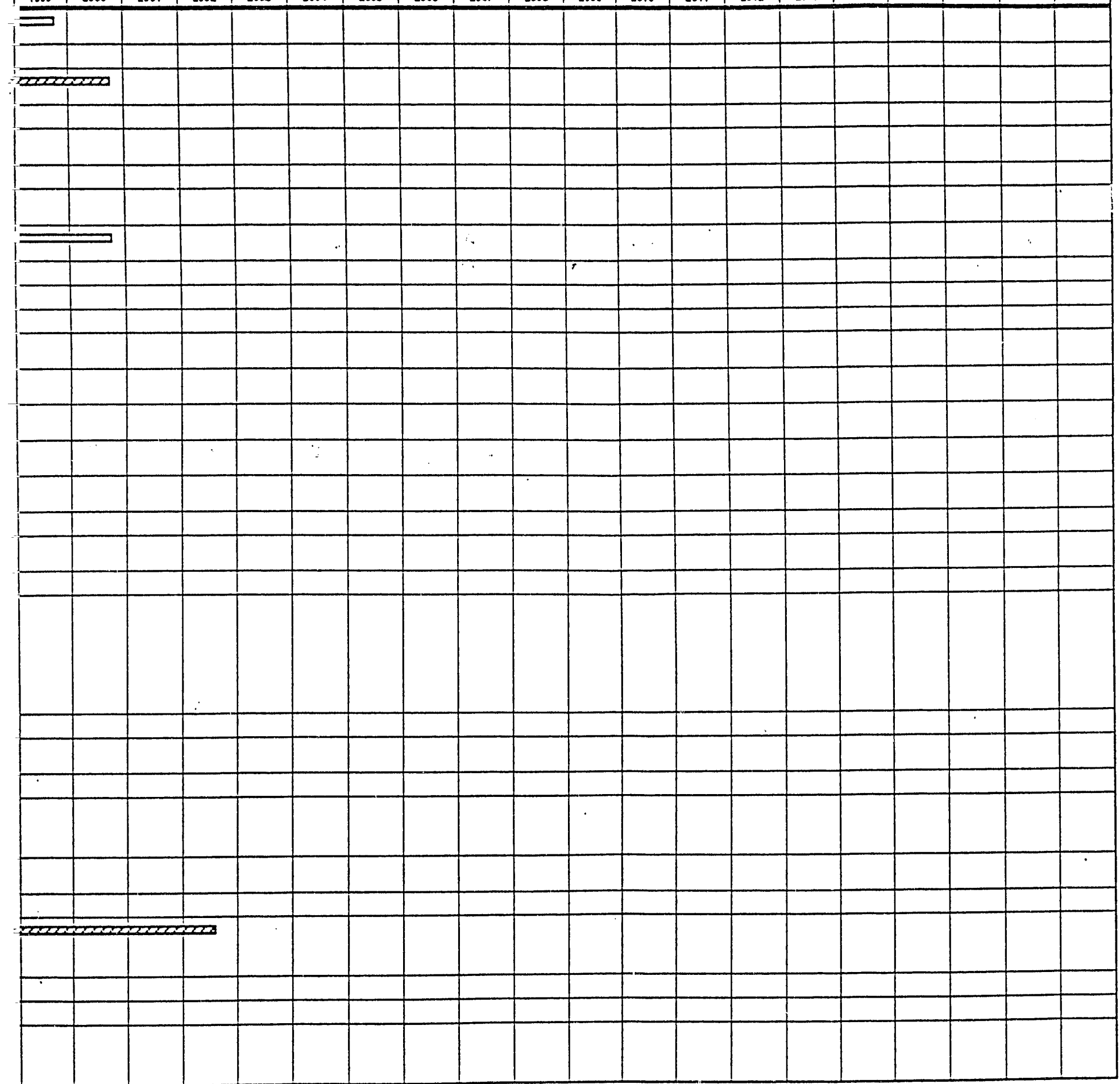


Issues Res

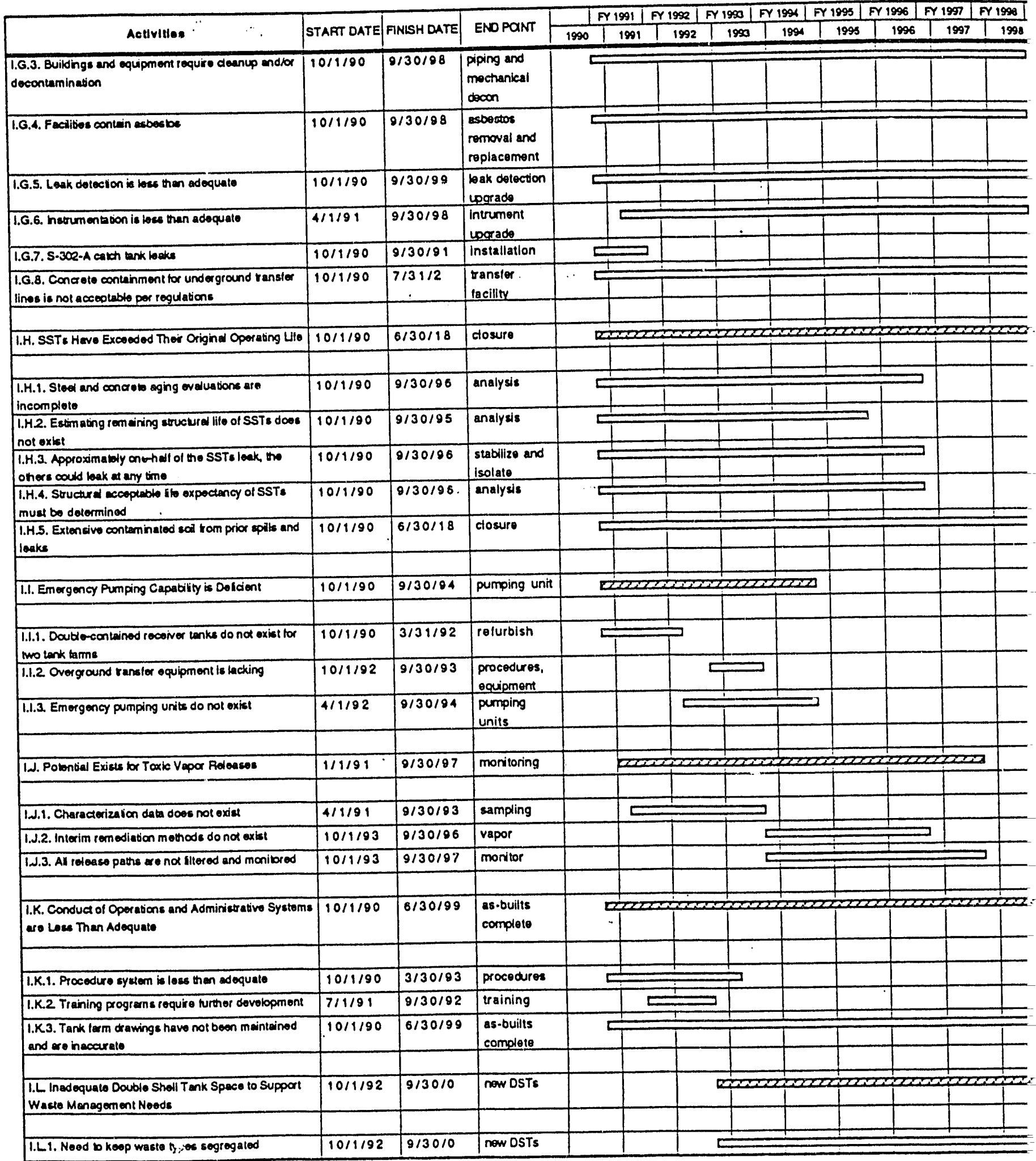


DOE/RL-90-48

Predecisional Draft

$4 / 5 / 91$

Tution Schedule

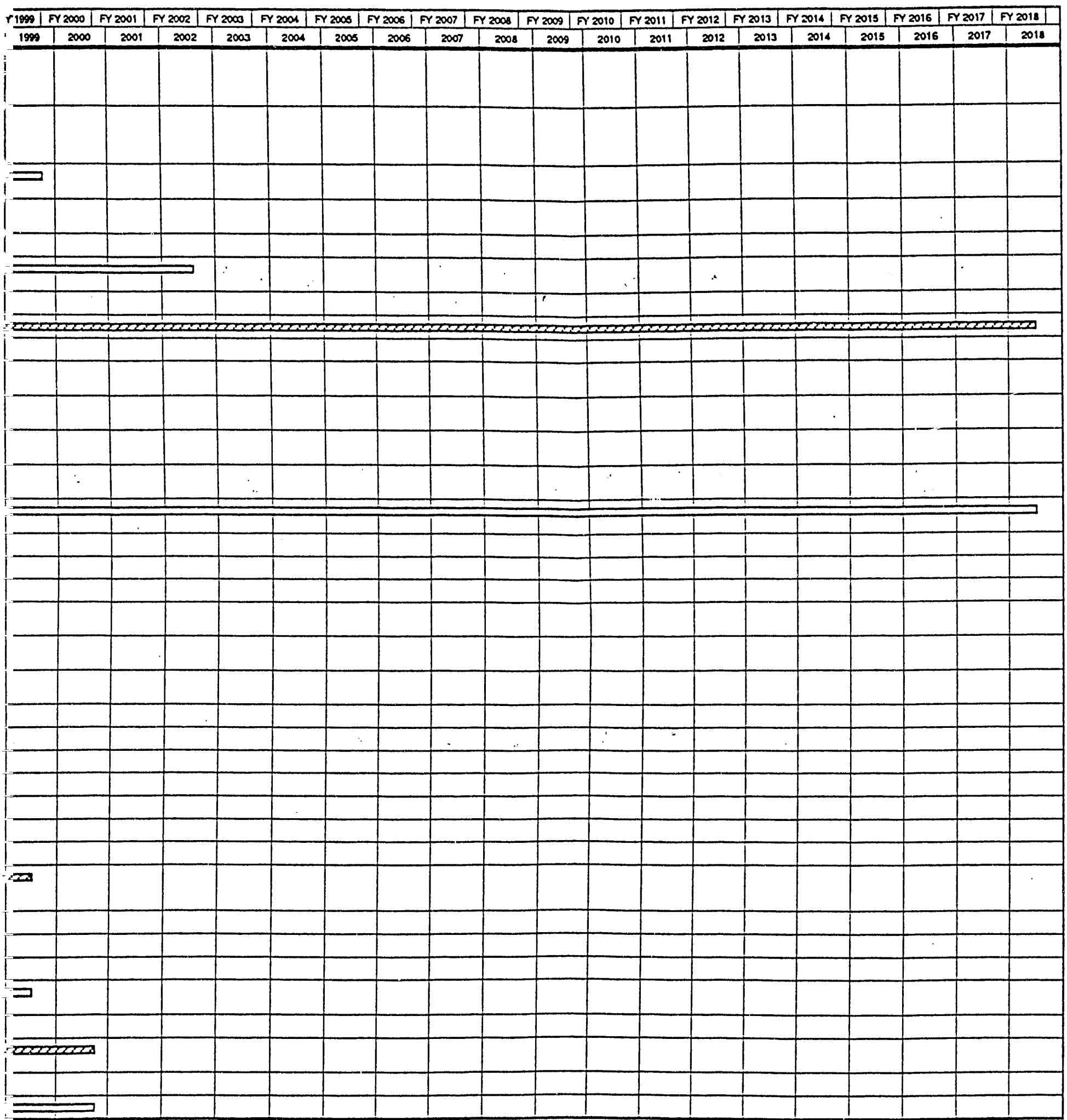


Issues $R$

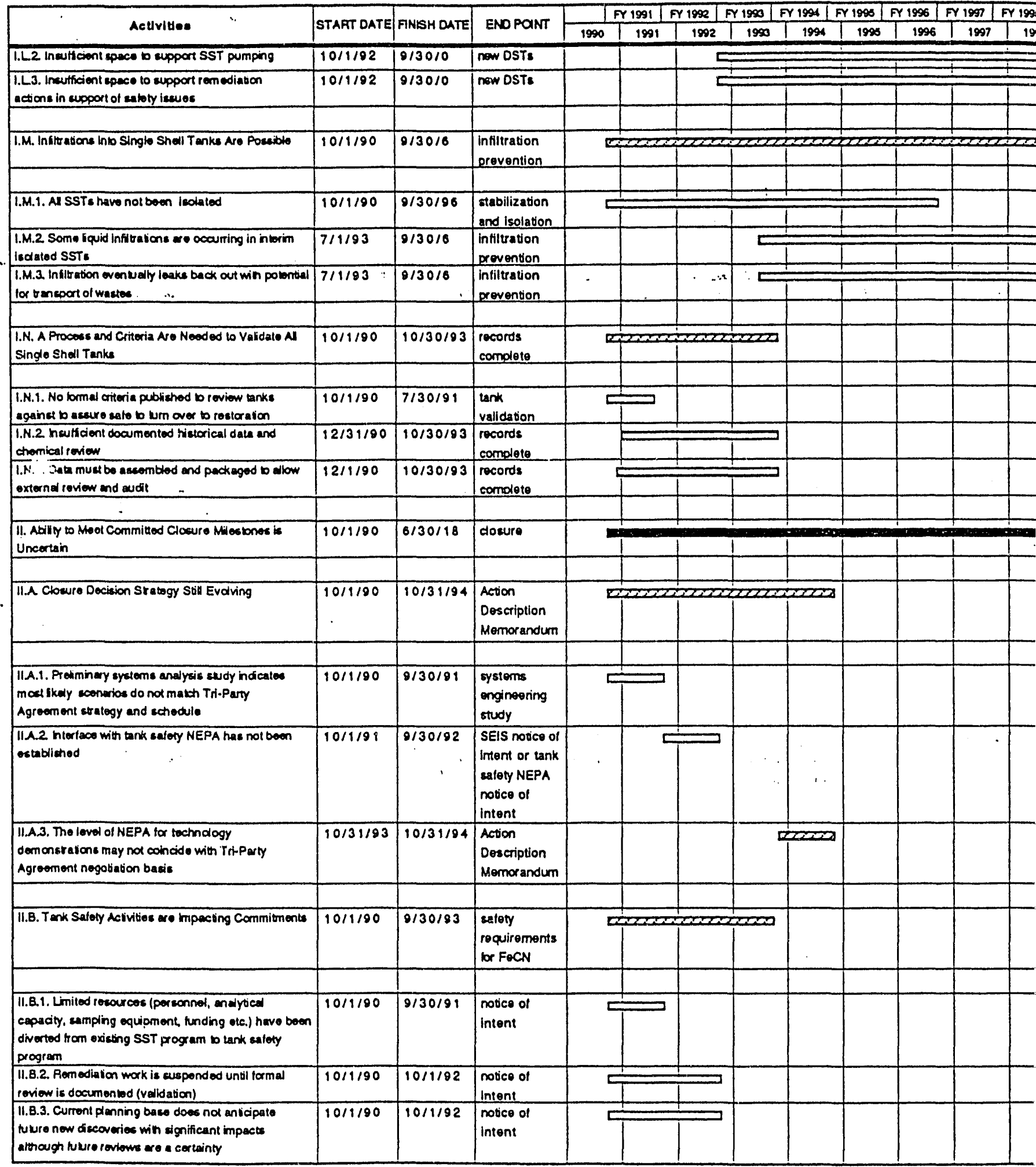


DOE/RL $-90-48$

Predecisional Draft

$4 / 5 / 91$

solution Schedule

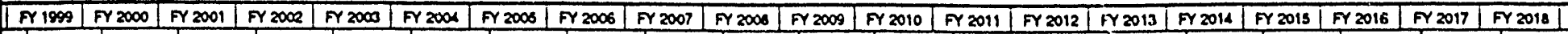
\begin{tabular}{|l|l|l|l|l|l|l|l|l|l|l|l|l|l|l|l|l|l|l|l|l|l|}
\hline 1908 & 2000 & 2001 & 2002 & 2003 & 2004 & 2008 & 2006 & 2007 & 2008 & 2009 & 2010 & 2011 & 2012 & 2013 & 2014 & 2013 & 2016 & 2017 & 2018 \\
\hline
\end{tabular}

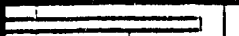

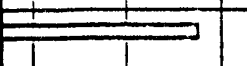

200000000000000000000000000000000
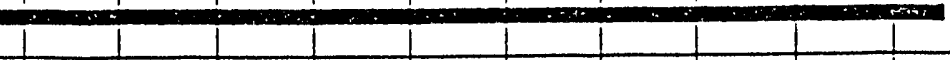
Issues $\operatorname{Re}$

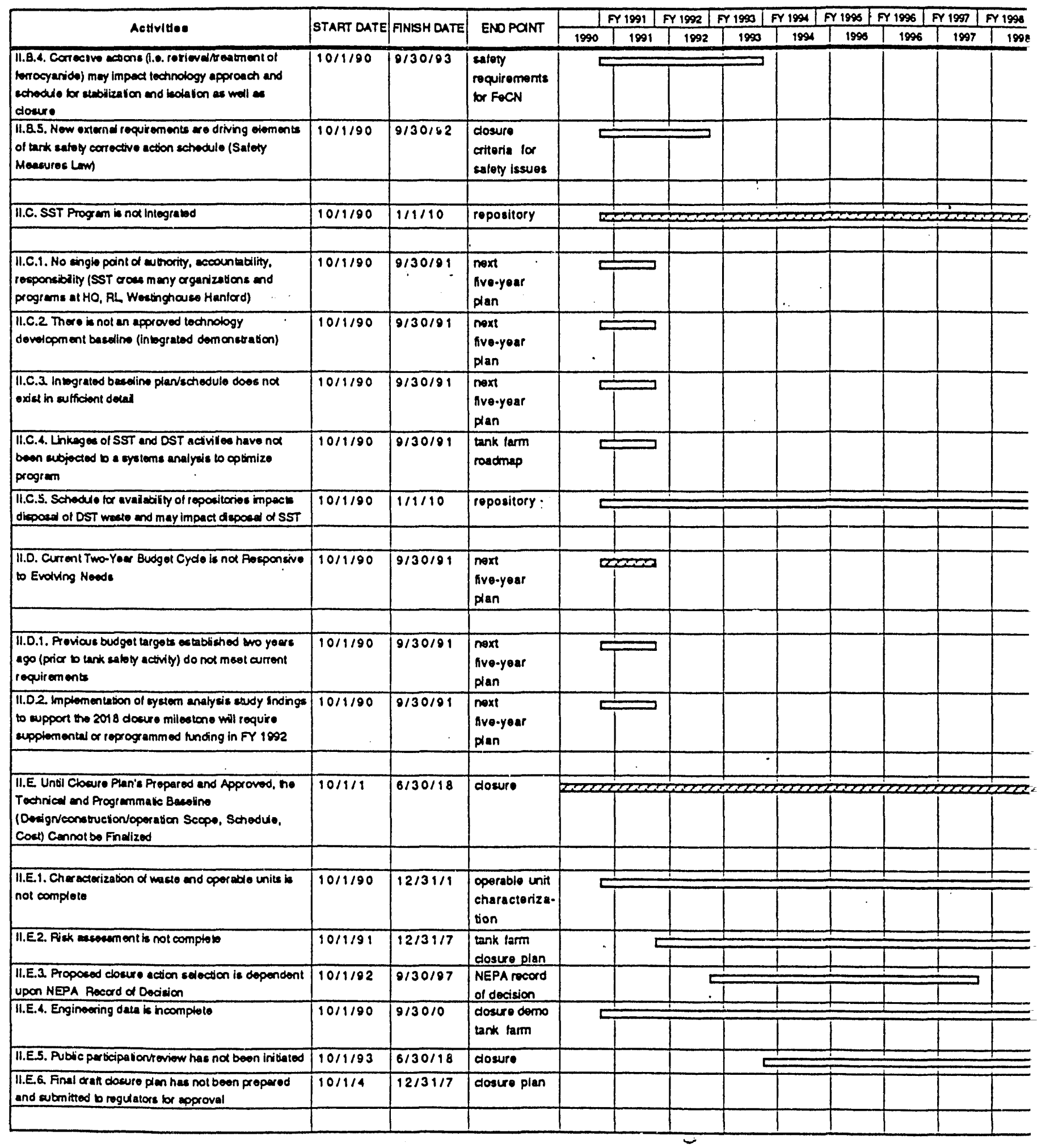


solution Schedule

DE: $8-50-48$

Prececisional Eraft

459 :

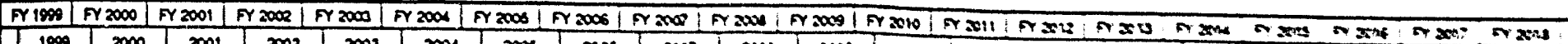

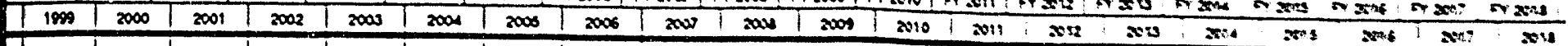
1 |

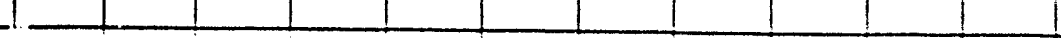

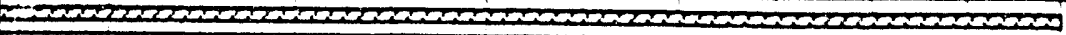

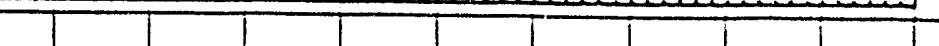

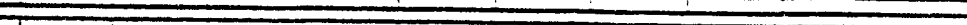

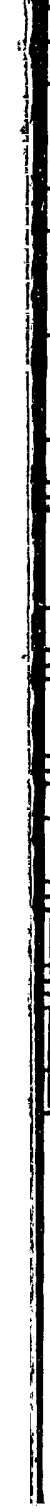


........Issues $R_{1}$

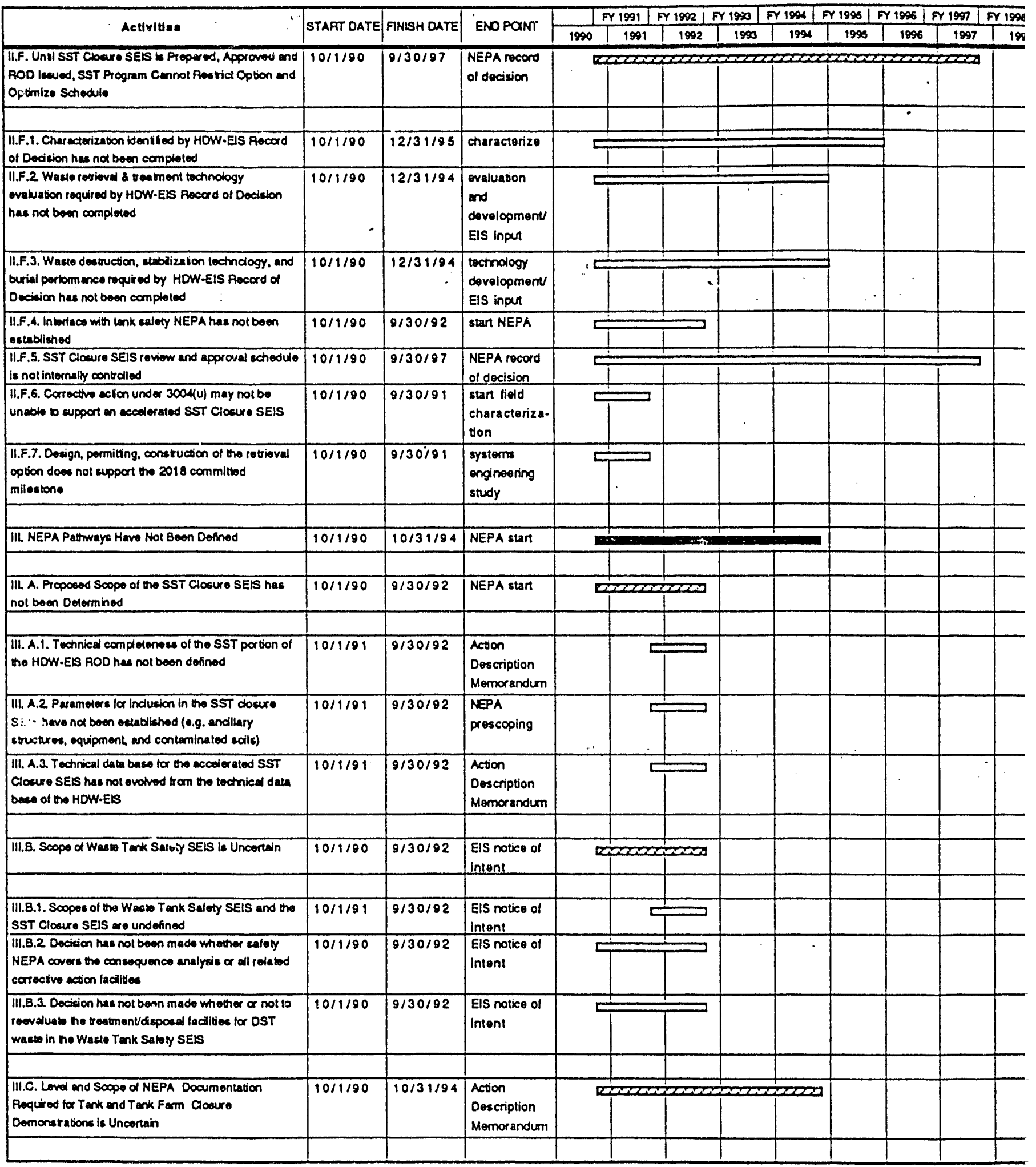




\section{DOE/RL - $90-48$}

Predecisional Draft $4 / 5 / 91$

solution Schedule ......

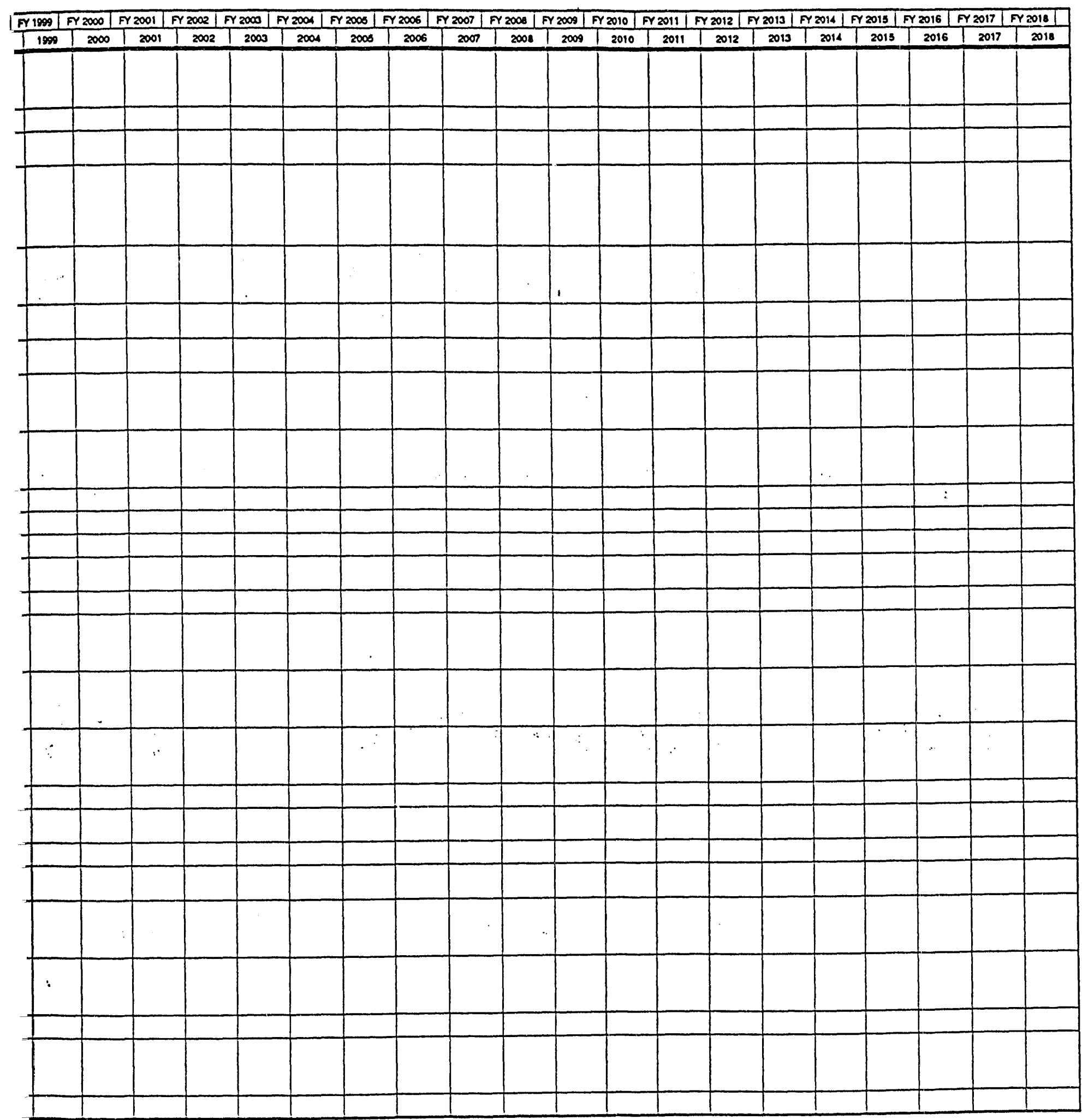


Issues Resi

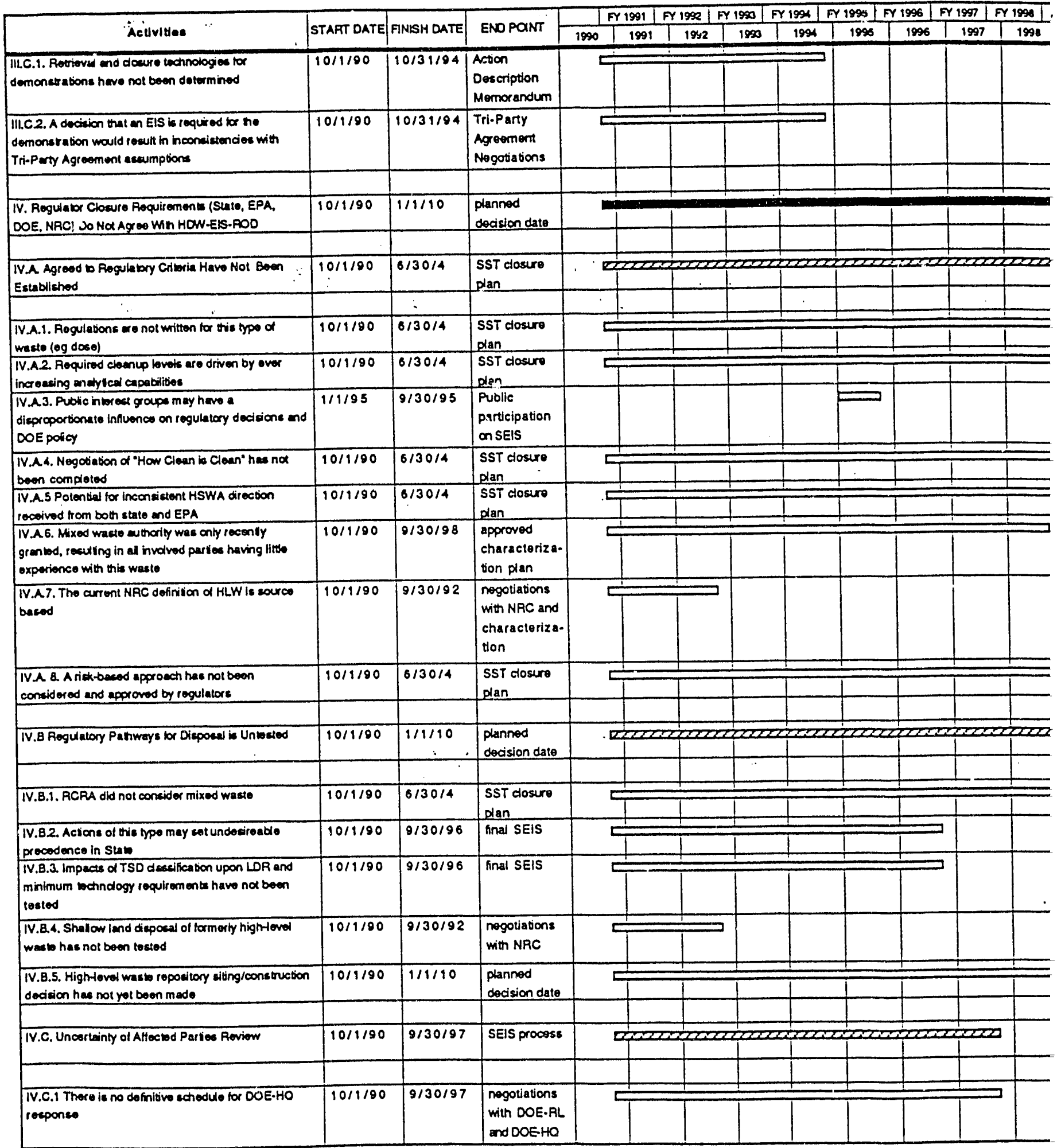


DOE/RL - $90-48$

Predecisional Draft

$4 / 5 / 91$

Iution Schedule

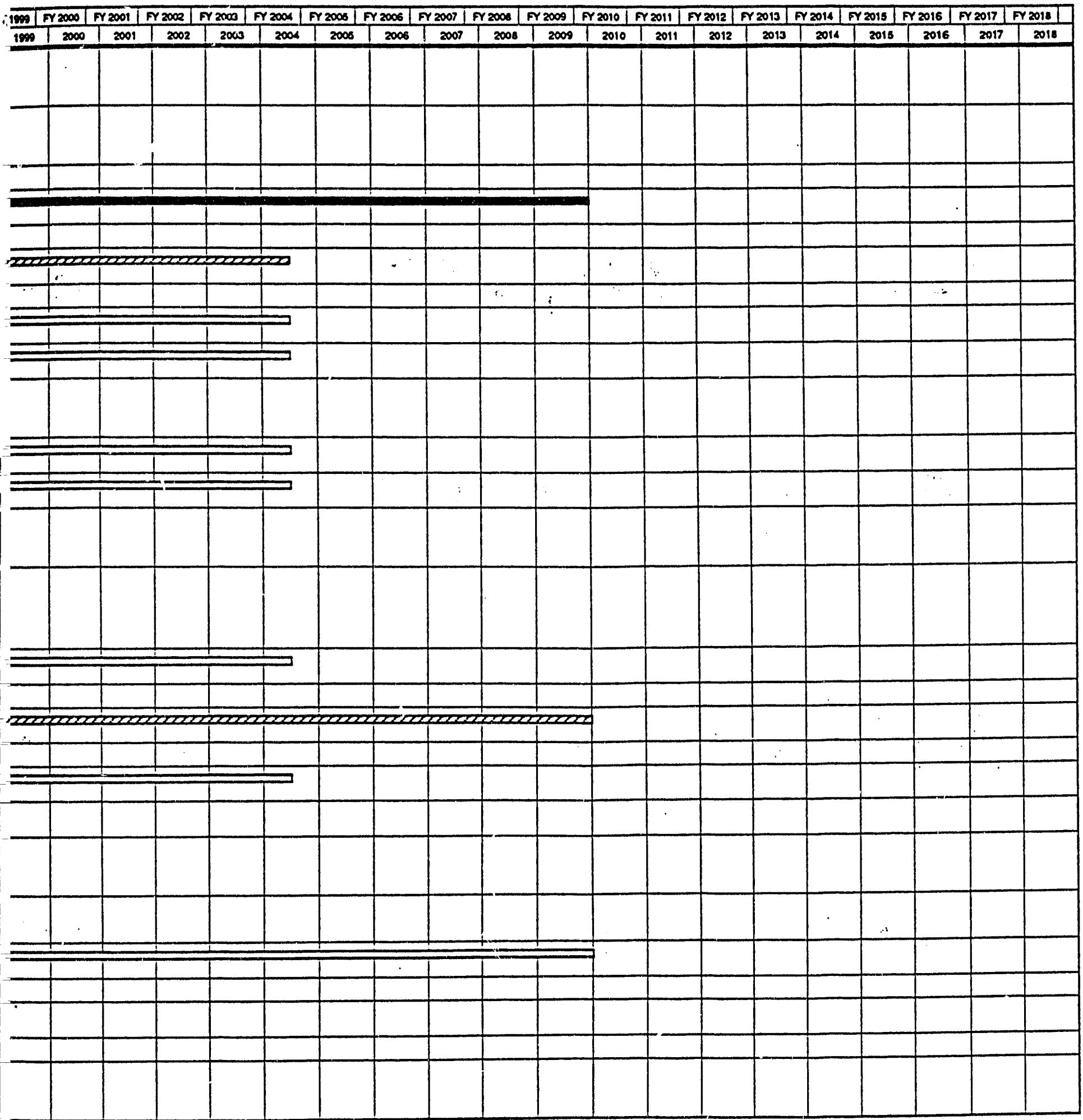


Issues Res

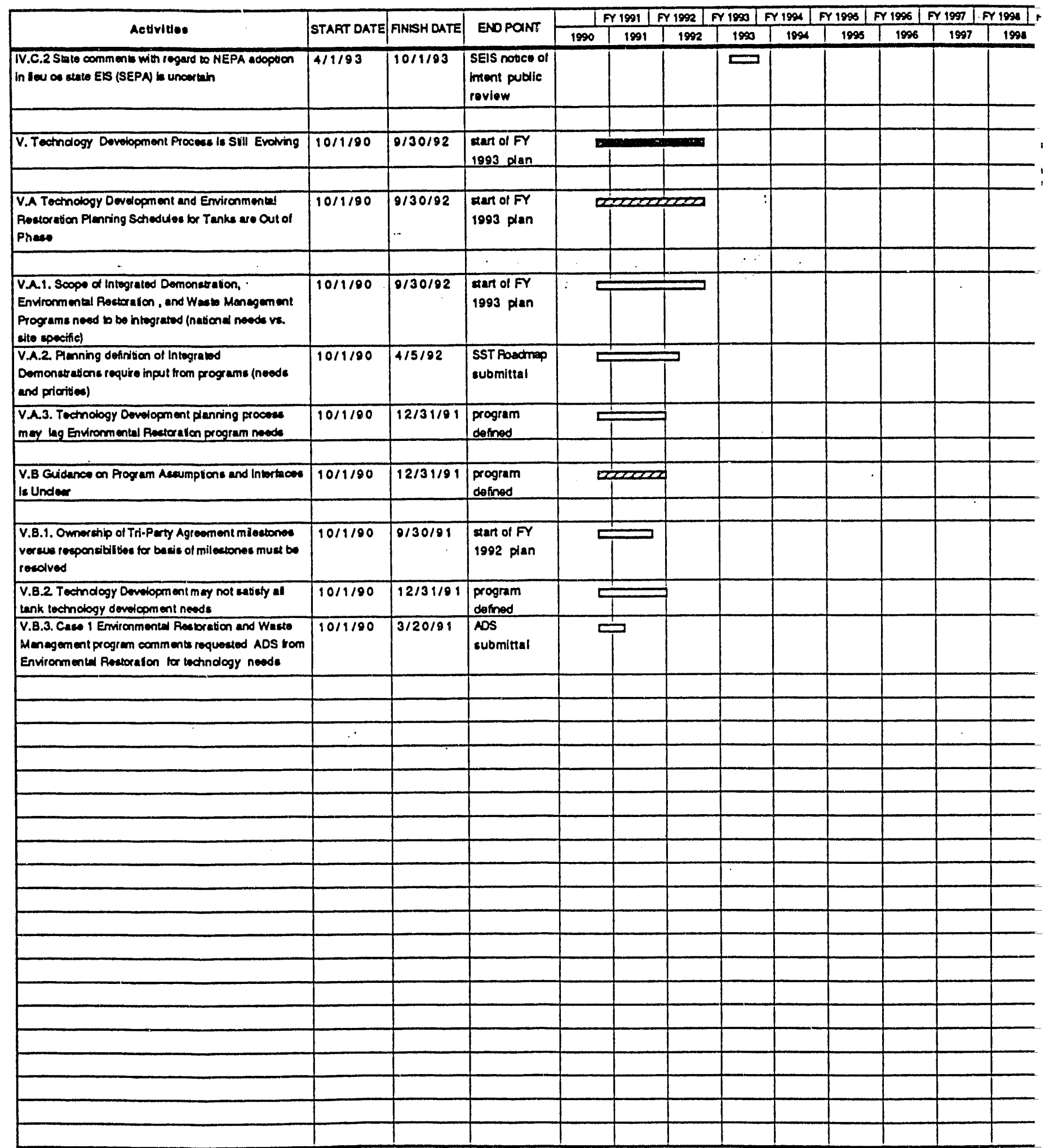


DOE/RL-90-48

Predecisional Draft

$4 / 5 / 91$

Iution Schedule

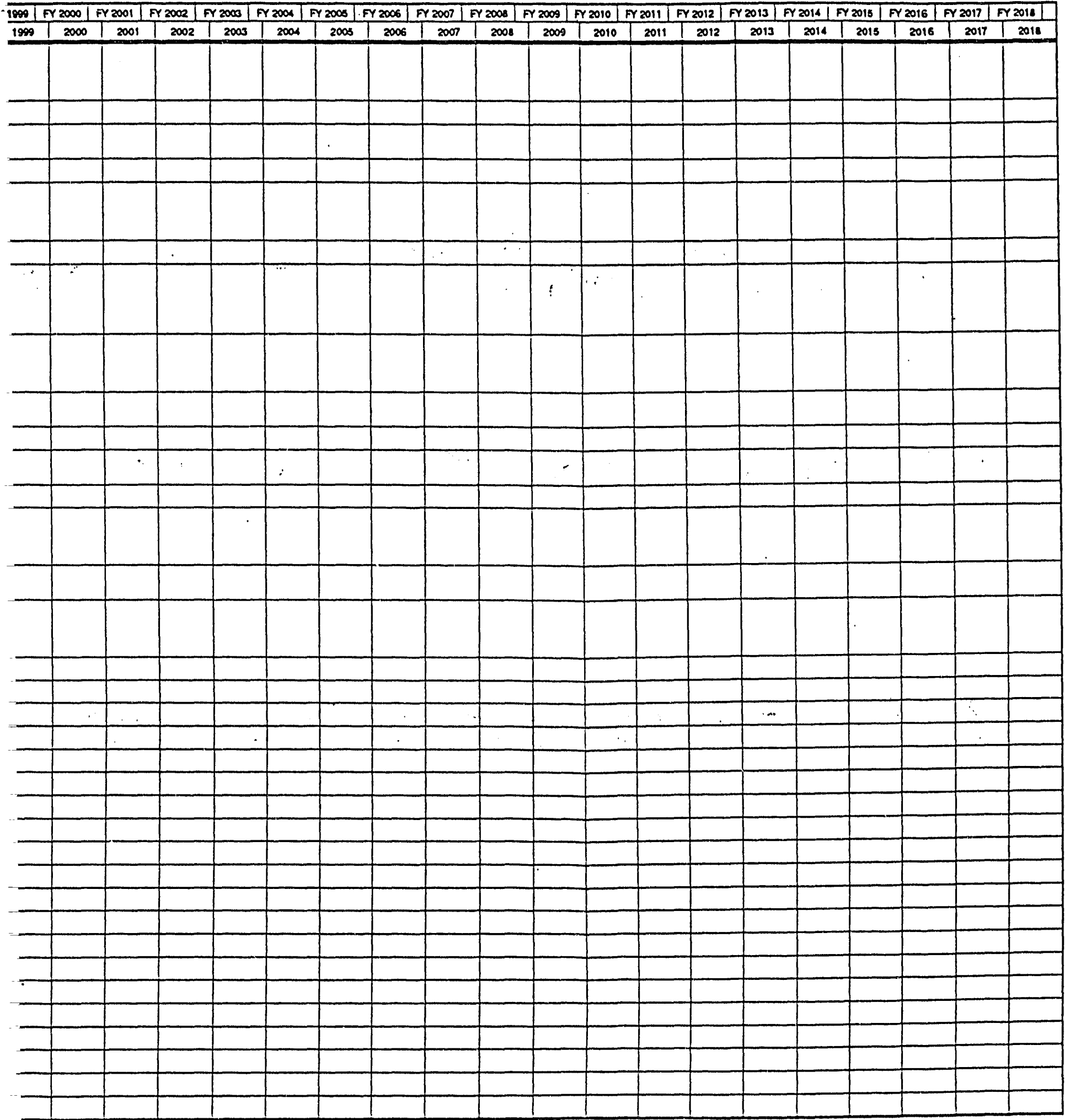


Desired Aci

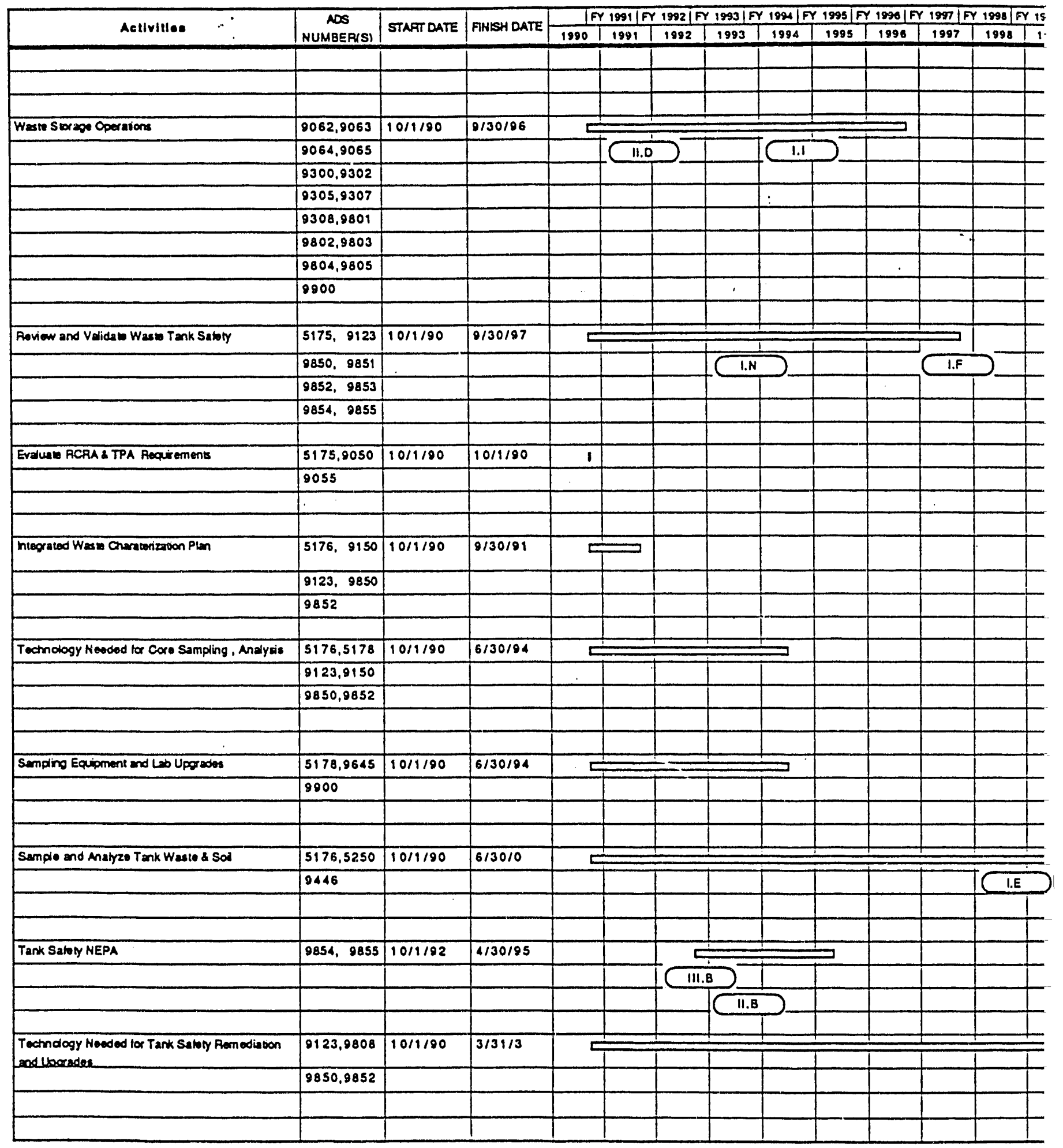


DOE/RL- $90-48$

Predecisional Draft

$4 / 5 / 91$

ivities Schedule

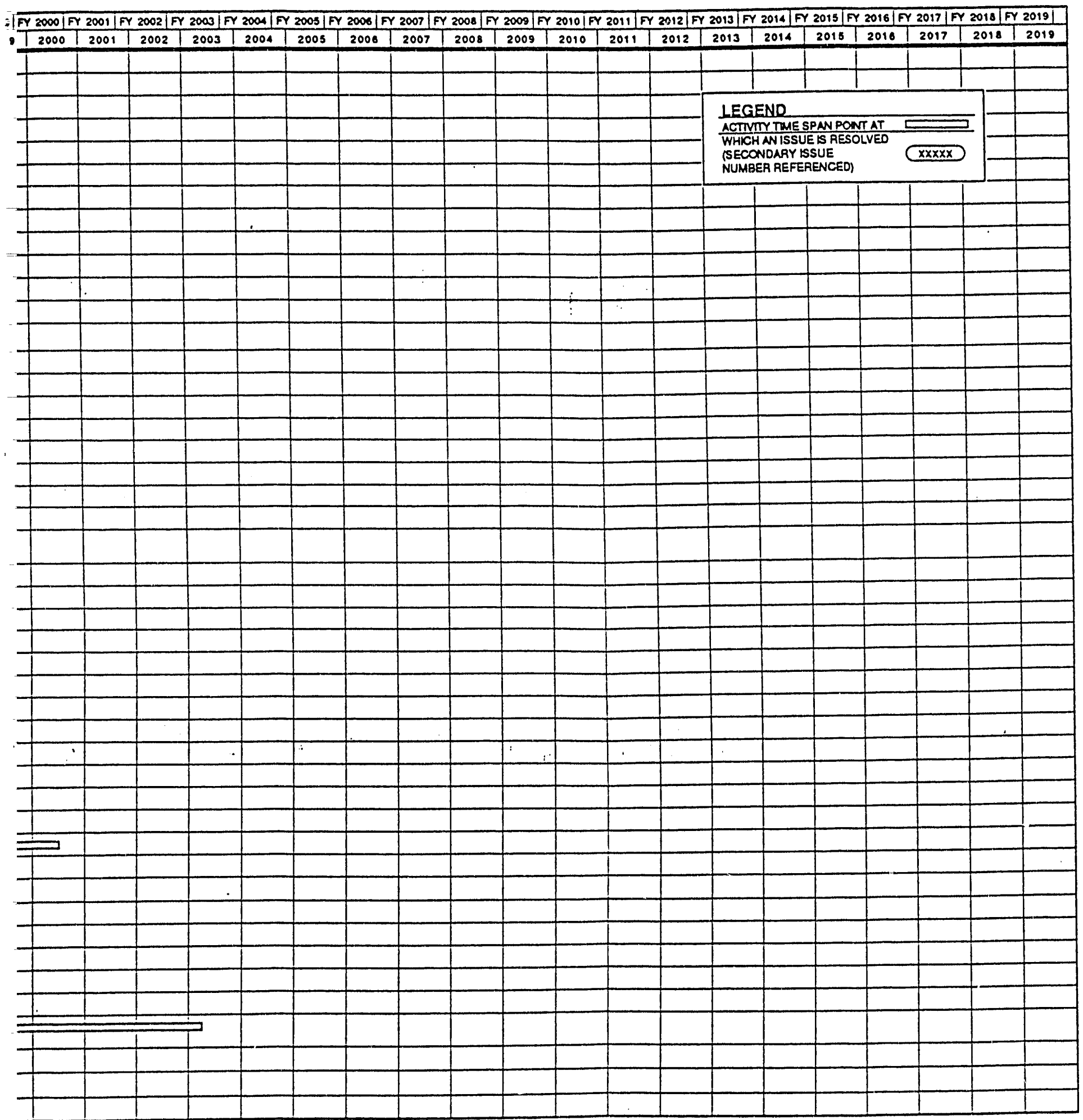


Desired AC

\begin{tabular}{|c|c|c|c|c|c|c|c|c|c|c|c|c|}
\hline \multirow{2}{*}{ Activitle: } & \multirow{2}{*}{$\begin{array}{c}\text { AOS } \\
\text { NUMBER(S) } \\
\end{array}$} & \multirow{2}{*}{ STAFT DATE } & \multirow{2}{*}{ FNISH DATE } & \multicolumn{9}{|c|}{ 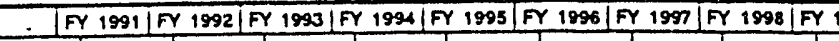 } \\
\hline & & & & 1990 & 1991 & 1992 & 1993 & 1994 & 1993 & 1996 & 1997 & 1998 \\
\hline Tenk Safery Remediaion and Rerofits (holudes & 5175,9123 & $10 / 1 / 90$ & $3 / 31 / 3$ & & & & & & & & & \\
\hline . & 9801,9802 & & & & & & & & & & 1.5 & \\
\hline & 9806.9850 & & & & & & & & & & & \\
\hline & 9851,9852 & & & & & & & & & & & \\
\hline & 9853 & & & & & & & & & & & \\
\hline & & & & & & & & & & & & \\
\hline $\begin{array}{l}\text { Technology Nood od tox Stabilization of High Heat } \\
\text { Tenks } 105 \mathrm{C}, 106 \mathrm{C}\end{array}$ & 9807 & $10 / 1 / 02$ & $9 / 30 / 94$ & & & & & כ & & & & \\
\hline & & & & & & & & & & & & \\
\hline$\therefore$ & & & 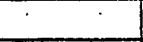 & & & & & & & $\cdot$ & & \\
\hline$\because$ & $\therefore$ & . & $\cdot$ & 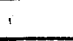 & - & $\therefore$ & $\therefore$ & & $\cdots$ & $\therefore$ & & \\
\hline Stabilize Tanks & 5175.5176 & $10 / 1 / 80$ & $9 / 30 / 95$ & & & & & & $\Longrightarrow$ & & & \\
\hline & 9300.9305 & & & & & & & & & & & \\
\hline & 9807 & & & & & & & & & & & \\
\hline & & & & & & & & & & & & \\
\hline Isolato SST: & 9305 & $10 / 1 / 90$ & $9 / 30 / 96$ & & $=$ & & & & & $\Longrightarrow$ & & \\
\hline & & & & & & & & & & & & \\
\hline & & & & & & & & & & & & \\
\hline - & & & & & & & & & & & & \\
\hline Sato Interim Storago & 9302,9307 & $10 / 1 / 96$ & $6 / 30 / 18$ & & & & & & & $=$ & & \\
\hline & & & & & & & & & & & & \\
\hline & & & & & & & & & & & & \\
\hline & & & & & & & & & & & & \\
\hline $\begin{array}{l}\text { Drak SST System Closure/Corroctive Action } \\
\text { Work Plan }(9 / 30 / 89)\end{array}$ & & $9 / 30 / 89$ & $9 / 30 / 89$ & & & & & & & & & \\
\hline & & & & & & & & & & & & \\
\hline & & & & & & & & & & & & \\
\hline & & & $12 / 31 / 94$ & & & & & & & & & \\
\hline Technology Neodod to Support Closuro SEIS & 5175 & $10 / 1 / 90$ & $12 / 31 / 94$ & & $=$ & & & 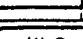 & & & & \\
\hline & & & & & & & & III.C & ) & & & \\
\hline$\therefore$ & &. & . & . & & 1.8 & & & & & & \\
\hline & & & $\Rightarrow$ & & & & & & . & & & \\
\hline Clocureses & 5175 & $10 / 1 / 82$ & $9 / 30 / 97$ & & & 5 & & & & & 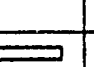 & \\
\hline & & & & & & $\pi 1.1$ & 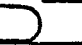 & & & & IIIF & \\
\hline & & & & & & III.A & & & & & N.C & \\
\hline & & & & & & & & & & & & \\
\hline Closure Plan & 5175 & $1 / 1 / 1$ & $12131 / 7$ & & & & & & & & & \\
\hline & & & & & & & & & & & & \\
\hline & & & & & & & & & & & & \\
\hline Dosign/PormitConstruct/Modity Closure & & $10 / 1 / 97$ & $9 / 30 / 8$ & & & & & & & & & \\
\hline & & & & & & & & & & & & \\
\hline & & & & & & & & & & & & \\
\hline & & & & & & & & & & & & \\
\hline Technology Noodod lor Insibu Trostment & & $10 / 1 / 90$ & $9 / 30 / 7$ & & & & & $-1+2+2$ & & & & \\
\hline & & & & & & & & & & & & \\
\hline & & & & & & & & & & & & \\
\hline
\end{tabular}


$D O E / R L-90-48$

Predecisional Draft

$4 / 5 / 91$

ivities Schedule

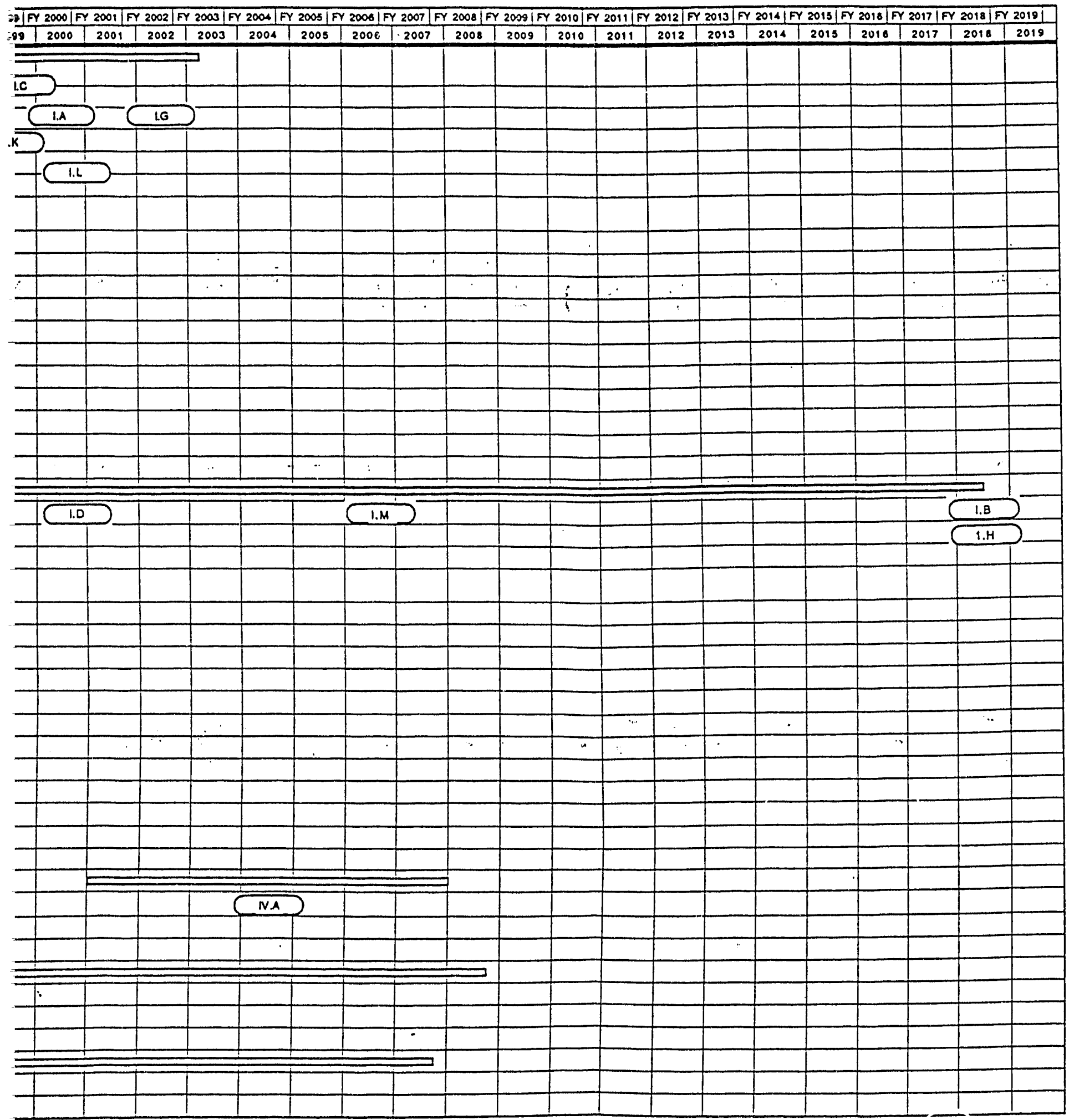


Desired Acti

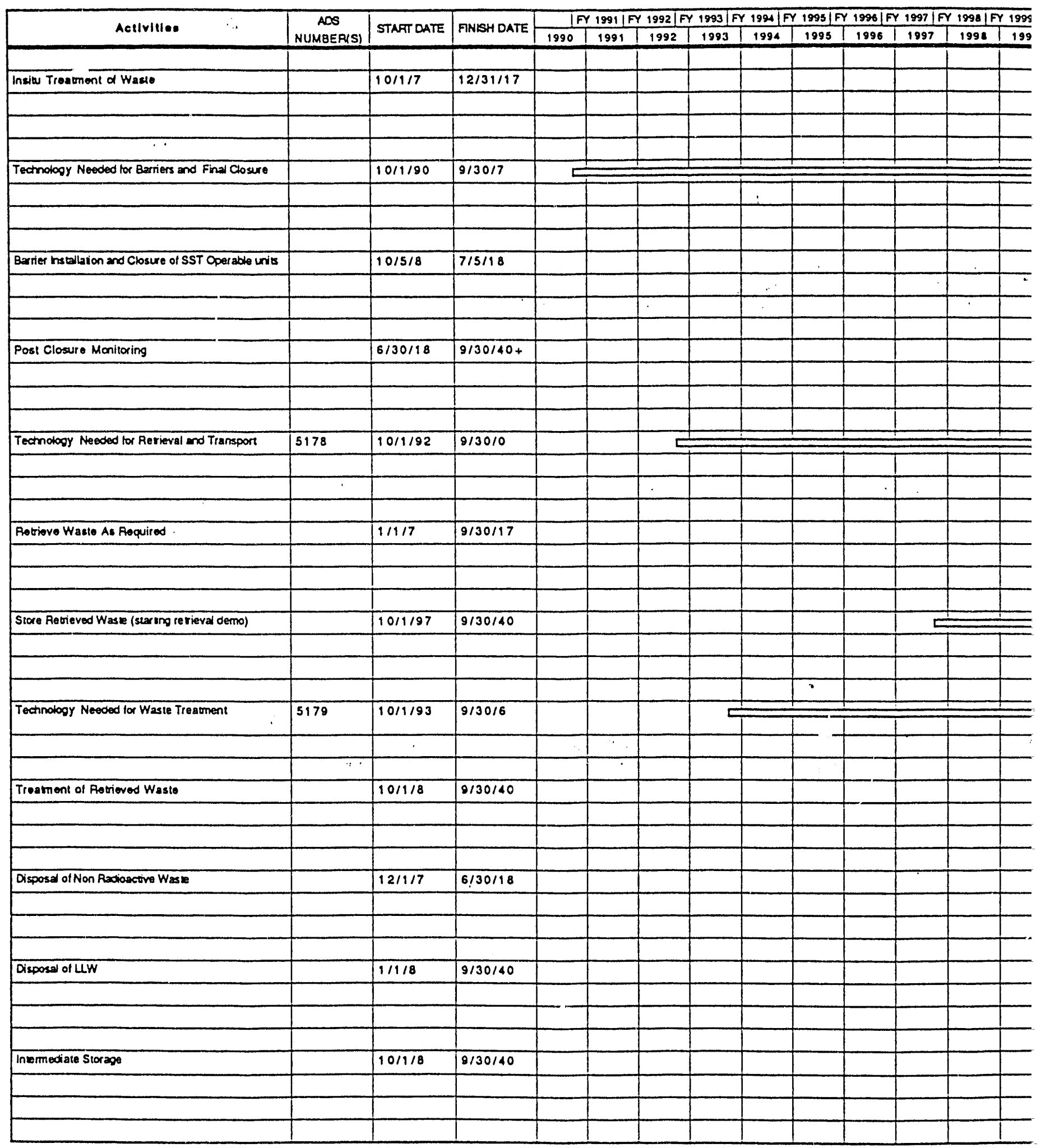


'ities Schedule

DOE/RL- $90-48$

Predecisional Draft

$4 / 5 / 91$

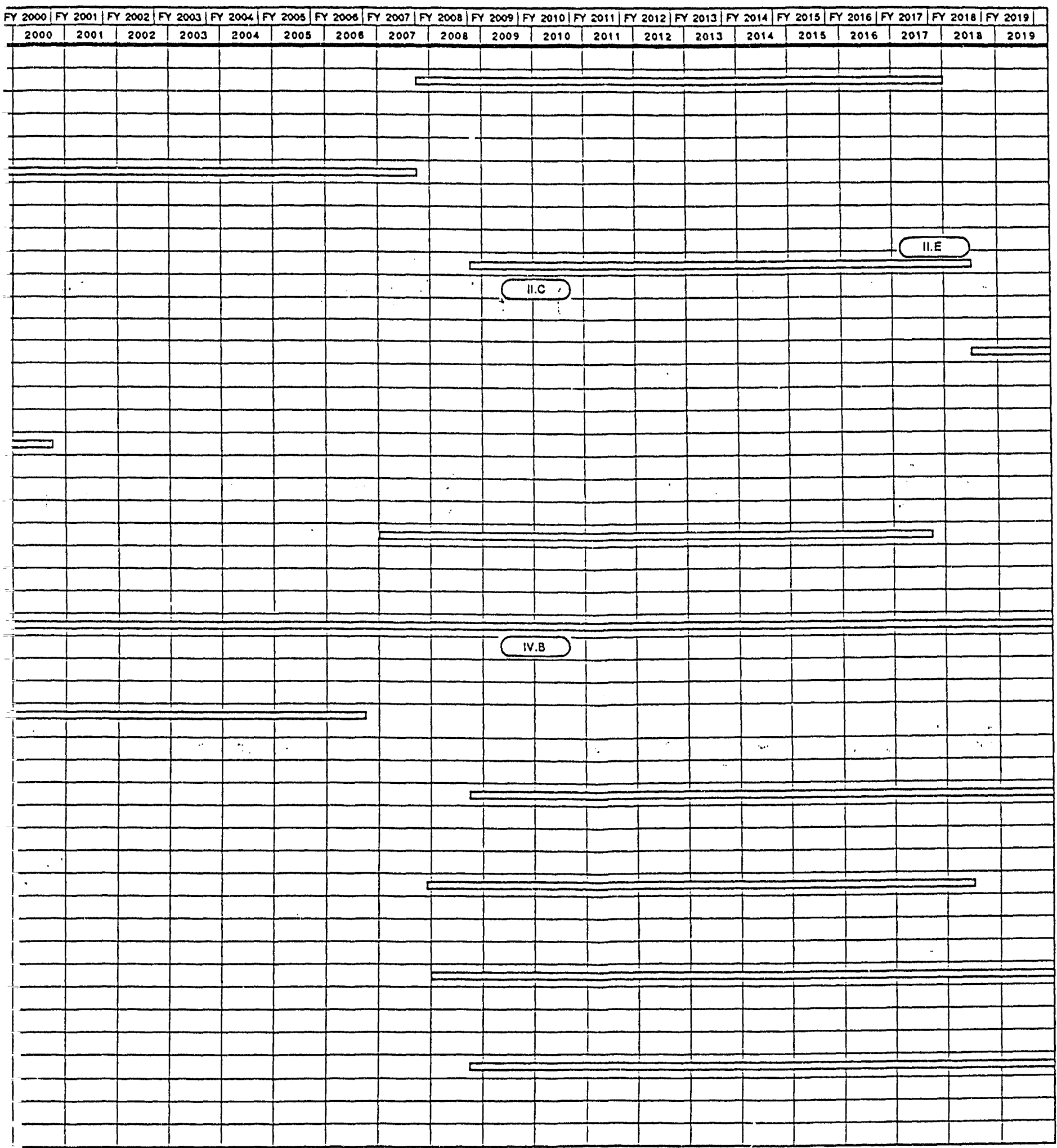


Desired Ac

\begin{tabular}{|c|c|c|c|c|c|c|c|c|c|c|c|c|}
\hline Activillos: & $\begin{array}{c}\text { NDS } \\
\text { NUMBERSI }\end{array}$ & STAFT DATE & FNSH DATEF & $\frac{T 1 F}{1990}$ & $\begin{array}{l}\text { Y1991/F } \\
1991\end{array}$ & 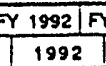 & & 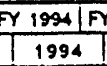 & & 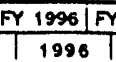 & & 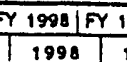 \\
\hline HLW Disposest & & $1 / 1 / 10$ & $9 / 36 / 40$ & & & & & & & - & & \\
\hline & & & & & & & & & & & & \\
\hline & & & & & & & & & & & & \\
\hline TAU Disposed & & $1 / 1 / 10$ & $9 / 30 / 40$ & & & & & & & - & & \\
\hline & & & & & & & & & & & & \\
\hline & & & & & & & & & & - & & \\
\hline \begin{tabular}{|l} 
Post Disposal Moritoring \\
\end{tabular} & & $111 / 8$ & $10 / 1 / 40+$ & & & & & & & - & & \\
\hline & & & & & & & & & & & & \\
\hline & & & & & & & & & & & & \\
\hline & & & & & & & & & & 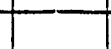 & 1 & \\
\hline & & & & & & & & & & & & \\
\hline & & & & & & & & & & & & \\
\hline & & & & & & & & & & & 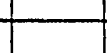 & \\
\hline & & & & & & & & & & & & \\
\hline & & & & & & & & & & & & \\
\hline & & & & & & & & & & 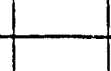 & & \\
\hline & & & & & & & & 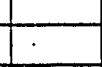 & & & 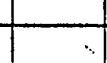 & \\
\hline & & & & & & & & & & - & E & \\
\hline & & & & & & & & 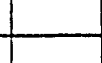 & & - & & \\
\hline & & & & & & & & & & & & \\
\hline & & & & & & & & & & & & \\
\hline & & & & & & & & & & & & \\
\hline & & & & & & & & & & & & \\
\hline & & & & & & & & & & & & \\
\hline & & & & & & & & & & & & \\
\hline & & & & & & & 1 & & & & 1 & \\
\hline & & & & & & & & & & & & \\
\hline & & & & & & & & & & & & \\
\hline & & & & & & & & & & & & \\
\hline & & & & & & & & & & & & \\
\hline & & & & & & & & & & & & \\
\hline & & & & & & & & & & & & \\
\hline & & & & & & & & & & & & \\
\hline & & & & & & & & & & & & \\
\hline & & & & & & & & & & & & - \\
\hline & & & & & & & & & & & 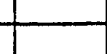 & 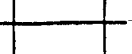 \\
\hline & & & & & & & & & & & $\pi$ & \\
\hline
\end{tabular}


$\mathrm{DOE} / \mathrm{RL}-90-48$

Predecisional Draft

$4 / 5 / 91$

ivities Schedule

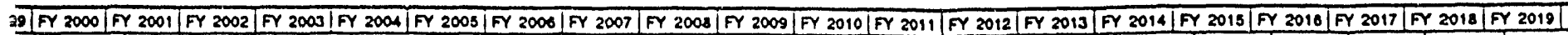

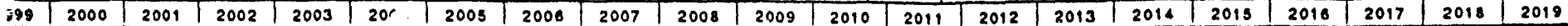

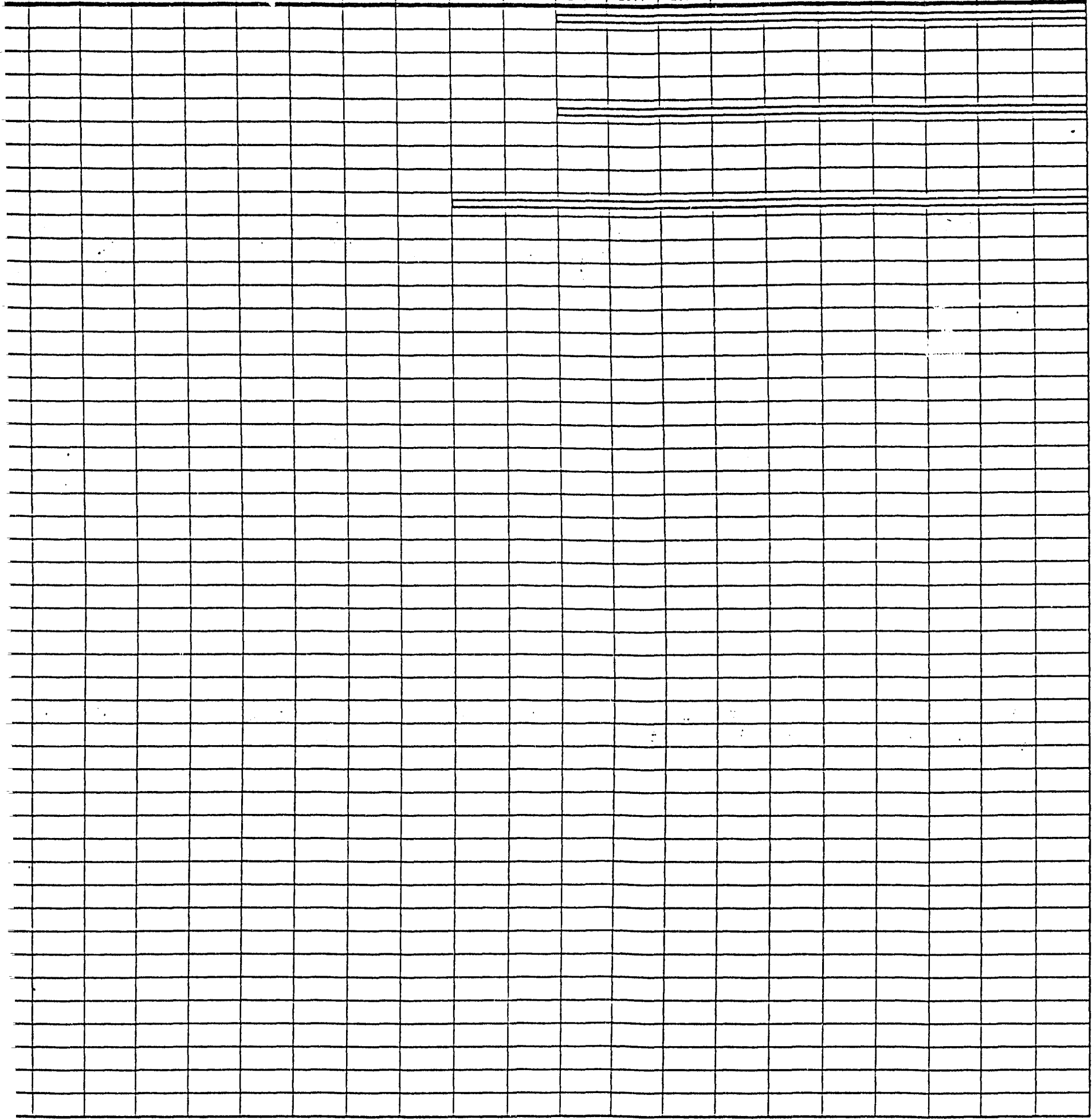




\subsection{HEADQUARTERS ISSUES}

This Chapter presents a list of all tertiary issues that have DOE-HQ listed as an actionee. For context, the applicable higher-level issue statements are included. A simple statement of what the needed action from DOE-HQ involves is included with each issue. More detail on the activity can be found in the issue analysis sheets in Chapter 7.0.

I. Current Storage Method for Waste is not Safe or Environmentally Sound.

B. Potentially explosive ferrocyanide mixture exists in some tanks.

1. Tanks contain large quantities of ferrocyanide compounds. DOE-HQ needs to review and approve the plan to address the ferrocyanide problem.

6. Corrective/remedial actions have not been identified/validated. DOE-HQ needs to review and approve ferrocyanide corrective actions.

7. Technology has not been demonstrated for retrieving waste. DOE-HQ needs to review and approve ferrocyanide waste retrieval methods and fund technology development activities.

I. Current storage method for waste is not safe or environmentally sound.

c. Potential reaction of organic and nitrate mixtures in some tanks.

1. No implementation management plan exists. DOE-HQ needs to approve the plan to address the organic and nitrate reaction problem.

4. Requirements have not been established, validated, and implemented for continued safe storage. DOE-HQ needs to review and approve requirements for any treatment needed to allow continued safe storage of organic and nitrate wastes.

5. Corrective/remedial action options are not identified/validated. DOE-HQ needs to review and approve corrective/remedial action options for organic and nitrate wastes. 
I. Current storage method for waste is not safe or environmentally sound.

D. Continue cooling required for high-heat generation tank 106-C.

1. Requirements have not been established, validated, and implemented. DOE-HQ needs to review and approve requirements for any alternatives to the partial retrieval approach to allow continued safe storage of high-heat tank waste.

I. Current storage method for waste is not safe or environmentally sound.

E. Characterization of tank contents is inadequate.

7. Technology for in situ characterization is not fully developed. DOE-HQ needs to approve funding for additional resources.

I. Current storage method for waste is not safe or environmentally sound.

L. Inadequate double-shell tank space to support waste management needs.

1. Insufficient space to support SST pumping. DOE-HQ needs to concur in any decision to and approve funding for any new storage capacity.

II. Ability to meet committed closure milestones is uncertain.

A. Closure decision strategy still evolving.

1. Preliminary systems analys is study indicates most likely scenarios do not match Tri-Party Agreement strategy and schedule. DOE-HQ needs to approve the technical basis for closure strategy and schedule.

2. Interface with tank safety NEPA has not been established. DOE-HQ needs to assist with determining the scope of the safety NEPA document.

3. The level of NEPA for technology demonstration may not coincide with Tri-Party Agreement negotiation basis. DOE-HQ needs to determine the appropriate level of NEPA documentation required for technology demonstrations. 
II. Ability to meet committed closure milestones is uncertain.

B. Tank safety activities are impacting commitments.

1. Limited resources (personnel, analytical capacity, sampling equipment, funding, etc.) have been diverted from existing SST program to tank safety program. DOE-HQ needs to provide needed funding for long-term resource needs.

2. Remediation work is suspended until formal review is documented (validation). DOE-HQ needs to review and approve tank safety work so that closure work can proceed.

4. Corrective action (i.e., retrievable/treatment of ferrocyanide) may impact technology approach and schedule for stabilization and isolation as well as closure. DOE-HQ needs to assist with major system acquisition issues and obtain support for needed funding. Also, DOE-HQ needs to el iminate the 2-year delay from completion of conceptual design to initiation of capital-funded work.

5. New external requirements are driving elements of tank safety corrective action schedule (Safety Measures Law). DOE-HQ needs to assist in assessment of impacts of new external requirements.

II. Ability to meet committed closure milestones is uncertain.

C. SST program is not integrated.

1. No single point of authority, accountability, responsibility (SST cross many organizations and programs at DOE-HQ, DOE-RL, and Westinghouse Hanford). DOE-HQ needs to participate in and approve in identification of a single point of authority.

2. There is not an approved technology development baseline (integrated demonstration). DOE-HQ needs to approve the Integrated Demonstration plan.

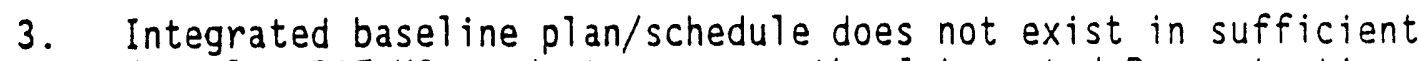
detail. DOE-HQ needs to approve the Integrated Demonstration plan.

5. Schedule for availability of repositories impacts disposal of DST waste and may impact disposal of SST waste. DOE-HQ needs to develop plans and schedule to provide repository space or interim storage for SST waste forms. 
II. Ability to meet committed closure milestones is uncertain.

D. Current 2-year budget cycle is not responsive to evolving needs.

2. Implementation of the SST System Engineering study findings to support the 2018 closure milestone will require supplemental or reprogrammed funding in FY 1992. DOE-HQ needs to provide the required funding level in FY 1992.

II. Ability to meet committed closure milestones is uncertain.

E. Until closure plans prepared and approved the technical and programmatic baseline (design/construction/operation scope, schedule, cost) cannot be finalized.

1. Characterization of waste and operable units is not complete. DOE-HQ needs to assist in establishing the level of characterization necessary to satisfy SST Closure SEIS technical and schedule requirement.

2. Risk assessment is not complete. DOE-HQ needs to assist in the process of reaching agreement on a risk-based approach.

5. Public participation/review has not been initiated. DOE-HQ needs to assist in and support a process of public participation in SST issues.

II. Ability to meet committed closure milestones is uncertain.

F. Until SST Closure SEIS is prepared, approved, and records of decision issued, SST program cannot restrict option and optimize schedule.

1. Characterization required by HDW-EIS record of decision has not been completed. DOE-HQ needs to approve the scope of SST characterization to satisfy NEPA requirements.

3. Waste destruction, stabilization technology, and burial performance required by the HOW-EIS record of decision has not been completed. DOE-HQ needs to assist in establishing criteria for the SEIS.

4. Interface with tank safety NEPA has not been established. DOE-HQ needs to assist in determining the scope of the tank safety NEPA requirements. 
5. SST Closure SEIS review and approval schedule is not internally controlled. DOE-HQ needs to provide timely review and action on NEPA documentation.

7. Design, permitting, and construction of the retrieval option does not support the 2018 committed milestone. DOE-HQ needs to assist with regulatory involvement in schedule impacts.

III. NEPA pathways have not been defined.

A. Proposed scope of the SST Closure SEIS has not been determined.

1. Technical completeness of the SST portions of the HOW-EIS record of decision has not been defined. DOE-HQ needs to determine if the guidelines of the HDW-EIS record of decision have been satisfied for determining adequate characterization, barrier performance, waste form stability, etc.

2. Parameters for inclusion in the SST Closure SEIS have not been established (e.g., anciliary structures, equipment, and contaminated soils). DOE-HQ needs to assist in determining the scope of the SST Closure SEIS.

III. NEPA pathways have not been defined.

B. Scope of Waste Tank Safety SEIS is uncertain.

1. Scopes of the Waste Tank Safety SEIS and the SST Closure SEIS are undefined. DOE-HQ needs to assist in determining the scope of the waste tank safety SEIS and issue the notice of intent.

2. Decision has not been made whether safety NEPA covers the consequence analysis or all related corrective action facilities. DOE-HQ needs to assist in determining the scope of the waste tank safety SEIS and issue in determining the scope of the waste tank safety SEIS and issue the notice of intent.

III. NEPA pathways have not been defined.

C. Level and scope of NEPA documentation required for tank and tank farm closure demonstration is uncertain.

1. Retrieval and closure technologies for demonstrations have not been determined. DOE-HQ needs to determine the appropriate level of NEPA documentation. 
2. A decision that an EIS is required for the demonstration would result in inconsistencies with Tri-Party Agreement assumptions. DOE-HQ needs to determine the level of NEPA documentation.

IV. Regulatory closure requirements (State, EPA, DOE, NRC) do not agree with HOW-EIS record of decision risk-based approach

A. Agreed-to regulatory criteria have not been established.

1. Regulations are not written for this type of waste

(e.g., dose). DOE-HQ needs to assist with any potential regulatory changes.

3. Public interest groups with specific agendas may have a disproportionate influence on regulatory decisions and DOE policy. DOE-HQ needs to support and assist with a technicallybased public meeting process.

4. Negotiations of "How Clean is Clean" has not been completed. DOE-HQ needs to participate in "How Clean is Clean" negotiations.

5. Potential for Inconsistent Hazardous and Solid Waste Amendments direction received from both State and EPA. DOE-HQ potentially needs to make a determination whether a sovereign immunity defense from state requirements is warranted.

6. Mixed waste authority was only recently granted, resulting in all involved parties having little experience with this waste. DOE-HQ needs to assist with potential mixed waste regulatory activities.

7. The current definition of high-level waste is source based and is being challenged by state regulators. DOE-HQ needs to assist with discussions with the NRC on high-level waste definition.

8. A risk-based approach has not been considered and approved by regulators. DOE-HQ needs to review RCRA for inconsistencies with $A E A$ requirements and negotiate modified procedures as indicated. 
IV. Regulatory closure requirements (State, EPA, DOE, NRC)

do not agree with HDW-EIS record of decision risk-based approach.

B. Regulatory pathway for disposal is untested.

1. RCRA did not consider mixed waste. DOE-HQ needs to review RCRA for inconsistencies with $A E A$ requirements and negotiate modified procedures as indicated.

3. Impacts of TSD classification upon LDR and minimum technology requirements have not been tested. DOE-HQ needs to participate in discussions with the NRC on whether SST waste is high-level waste and potentially determine if a new definition, based on activity, is justified.

4. Shallow land disposal of formerly high-level waste has not been tested.

5. High-level waste repository siting/construction decision has not yet been made. DOE-HQ needs to proceed with high-level waste repository activities.

IV. Regulatory closure requirements (State, EPA, DOE, NRC) do not agree with HDW-EIS record of decision risk-based approach.

C. Uncertainty of affected parties review.

1. There is no definitive schedule for DOE-HQ response. DOE-HQ needs to prepare and meet schedules for responses and decisions on NEPA documentation. DOE-HQ needs to participate in negotiations with Ecology on the SST Closure SEIS.

2. State comment with regard to NEPA adoption in lieu of State EIS (SEPA) is uncertain.

V. Technology development process is still evolving.

A. Technology development and environmental restoration and waste management planning schedules for tanks are out of phase.

1. Scope of Integrated Demonstration and Environmental Restoration Programs need to be integrated (nationa! needs versus sitespecific. DOE-HQ needs to assist in determining the scope integrated demonstrations.

2. Planning definition of Integrated Demonstrations require site input from programs (needs.and priorities). DOE-HQ needs to 
assist in incorporation of roadmap needs into the technology program.

3. Technology development planning process may lag site Environmental Restoration and Waste Management Program needs. DOE-RL needs to approve a field office decision on how shortterm technology needs will be met.

V. Technology development process is still evolving.

B. Guidance on program assumptions and interfaces is unclear.

1. Ownership of Tri-Party Agreement milestones versus responsibilities for basis of milestones must be resolved. DOE-HQ needs to approve the Technology Development Plan.

3. Case 1 Environmental Restoration Program comments requested ADSs from Environmental Restoration for technology and interface needs. DOE-HQ needs to approve a field office position paper on environmental restoration versus technology development furding for the Underground Storage Tank Integrated Demonstration Program. 


\subsection{REFERENCES}

1. Environmental Restoration and Waste Management Roadmap Methodology Document, Predecisional Draft, Change 1, October 1990, U.S. Department of Energy-Headquarters, Washington, D.C.

2. Hanford Federal Facility Agreement and.Consent Order, as amended, 1990, Washington State Department of Ecology, U.S. Environmental Protection Agency, and U.S. Department of Energy, Olympia, Washington.

3. The Hanford Site Environmental Restoration and Waste Management Five-Year Plan Activity Data Sheets, DOE/RL 89-17, Revision 1, U.S. Department of Energy-Richland Operations Office, Richland, Washington.

4. The Hanford Site Environmental Restoration and Waste Management Five-Year Plan Activity Data Sheets, DOE/RL 89-17, U.S. Department of EnergyRichland Operations Office, Richland, Washington.

5. The Waste Characterization Plan for the Hanford Site Single-Shell Tanks, WHC-EP-0210, December 1990, Westinghouse Hanford Company, Richland, Washington.

6. Final Environmental Impact Statement, Disposal of Hanford Defense High-Level, Transuranic, and Tank Waste, Hanford Site, Richland, Washington, DOE/EIS-0113, 5 Volumes, U.S. Department of Energy, Washington, D.C.

7. Letter, James D. Watkins to J.T. Conway, Defense Nuclear Facilities Safety Board, December 3, 1990.

8. Hanford Site Preliminary Action Plan, DOE/EH-NNNN (Draft), January 1991, U.S. Department of Energy-Richland Operations Office, Richland, Washington. 
1

1

This page intentionally left blank. 
$\nabla$
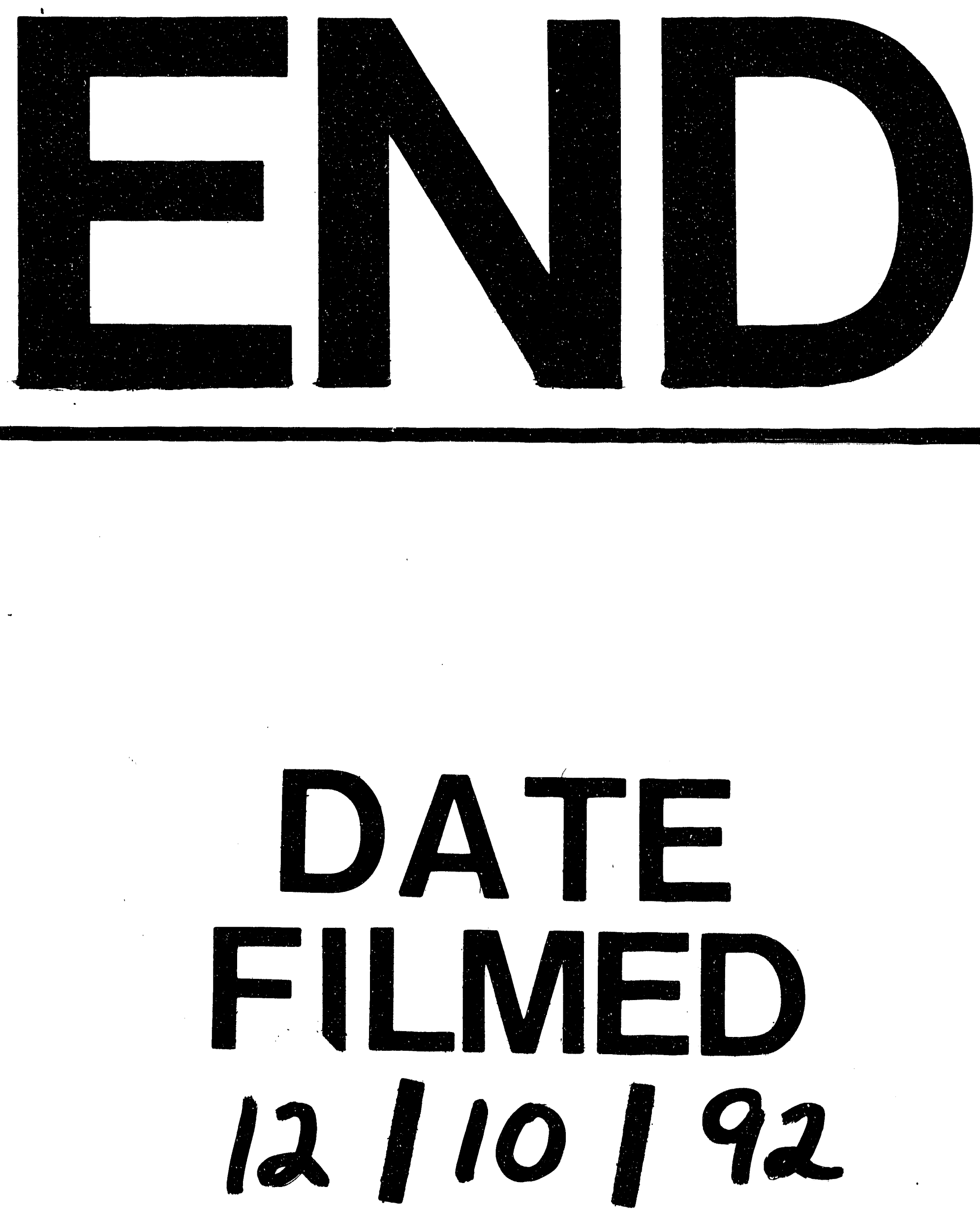
